Preprint typeset in JHEP style - HYPER VERSION

\title{
An effective theory for jet propagation in dense QCD matter: jet broadening and medium-induced bremsstrahlung
}

\author{
Grigory Ovanesyan, Ivan Vitev \\ Los Alamos National Laboratory, Theoretical Division, MS B283, Los Alamos, NM 87545, U.S.A. \\ E-mail: ovanesyan@lanl.gov, ivitev@lanl.gov
}

\begin{abstract}
Two effects, jet broadening and gluon bremsstrahlung induced by the propagation of a highly energetic quark in dense QCD matter, are reconsidered from effective theory point of view. We modify the standard Soft Collinear Effective Theory (SCET) Lagrangian to include Glauber modes, which are needed to implement the interactions between the medium and the collinear fields. We derive the Feynman rules for this Lagrangian and show that it is invariant under soft and collinear gauge transformations. We find that the newly constructed theory $\mathrm{SCET}_{\mathrm{G}}$ recovers exactly the general result for the transverse momentum broadening of jets. In the limit where the radiated gluons are significantly less energetic than the parent quark, we obtain a jet energy-loss kernel identical to the one discussed in the reaction operator approach to parton propagation in matter. In the framework of $\mathrm{SCET}_{\mathrm{G}}$ we present results for the fully-differential bremsstrahlung spectrum for both the incoherent and the Landau-PomeranchunkMigdal suppressed regimes beyond the soft-gluon approximation. Gauge invariance of the physics results is demonstrated explicitly by performing the calculations in both the light-cone and covariant $R_{\xi}$ gauges. We also show how the process-dependent medium-induced radiative corrections factorize from the jet production cross section on the example of the quark jets considered here.
\end{abstract}




\section{Contents}

1. Introduction $r$

2. A brief overview of SCET 3

3. Kinematics of the in-medium jet interactions 4

4. An effective theory for jet propagation in QCD matter: $\operatorname{SCET}_{\mathrm{G}} \quad 7$

4.1 The $\mathrm{SCET}_{\mathrm{G}}$ Lagrangian for different sources and gauges $\quad 8$

4.2 Gauge invariance of $\mathcal{L}_{\mathrm{G}} \quad 12$

5. Jet broadening $r$

$\begin{array}{lll}5.1 & \text { Quark jet } & 14\end{array}$

$\begin{array}{lll}5.2 & \text { Gluon jet } & 16\end{array}$

$\begin{array}{lr}\text { 6. Medium-induced bremsstrahlung } & 18\end{array}$

6.1 Obtaining the Altarelli-Parisi splitting function in SCET 19

6.2 Single Born amplitudes in $\mathrm{SCET}_{\mathrm{G}} \quad 21$

6.3 Double Born amplitudes in $\mathrm{SCET}_{\mathrm{G}} \quad 22$

7. Gauge invariance of the jet broadening and the medium-induced bremsstrahlung results 25

7.1 Hybrid gauge $A_{c}^{+}=0, R_{\xi}\left(A_{g}\right) \quad 25$

7.2 Positive light-cone gauge $A_{c, g}^{+}=0 \quad 26$

8. Identifying the in-medium jet evolution kernels 29

8.1 Reaction operator for collisional interactions 30

8.2 Reaction operator for medium-induced gluon bremsstrahlung 31

9. Bremsstrahlung: beyond the soft gluon approximation 33

9.1 Incoherent radiation 33

9.2 Final-state radiation and the Landau-Pomeranchuk-Migdal effect 35

$\begin{array}{ll}\text { 10. Conclusions } & 38\end{array}$

$\begin{array}{lr}\text { A. Light-cone notation } & 39\end{array}$

B. Kinematics in the laboratory frame $\quad 39$

C. Feynman rules of $\mathrm{SCET}_{\mathrm{G}} \quad 40$

D. Longitudinal integrals $\quad 42$

$\begin{array}{ll}\text { D.1 Jet broadening } & 42\end{array}$

D.2 Radiative energy loss 43 


\section{Introduction}

The start up of the Large Hadron Collider (LHC) has reinvigorated strong theoretical interest in the physics of energetic jets $[1,2]$. At present, many of the new developments in the Quantum Chromodynamics (QCD) theory of hard jet and particle production are motivated by the need for precise evaluation of the Standard Model background to signature processes for new physics [3, 4, 5]. Such accuracy relies on factorization theorems $[6,7]$ that allow to separate the perturbative hard scattering part of the cross section from the non-perturbative parton distribution and fragmentation functions.

Recently, Soft Collinear Effective Theory (SCET) $[8,9,10,11]$ has emerged as a powerful new tool to address large $Q^{2}$ processes in lepton-lepton $\left(\ell^{+}+\ell^{-}\right)$, lepton-hadron $\left(\ell^{+}+p\right)$ and hadron-hadron $(p+p$ or $p+\bar{p}$ ) collisions. SCET in conjunction with QCD factorization, which has been proven in this framework for a number of processes $[12,13,14,15,16,17,18,19]$, is particularly suited to improving the precision of multi-scale calculations ${ }^{1}$ through the resummation of large Sudakov type logarithms [17, 20, 21, 22, 23, 24].

An important multi-scale problem is presented by the production of jets in reactions with large nuclei, such as lepton-nucleus $(\ell+A)$, proton-nucleus $(p+A)$ and nucleus-nucleus $(A+A)$ reactions. In these processes the energetic quarks and gluons must traverse a region of dense nuclear matter of $\mathcal{O}(5 \mathrm{fm})$ and their interactions in the medium induce broadening and a new type of radiative corrections that can significantly alter the corresponding jet cross sections and shapes [25, 26, 27, 28]. Preliminary results from the Relativistic Heavy Ion Collider (RHIC) and new results from the LHC indicate that these effects may indeed be observable in A+A collisions [29, 30, 31, 32, 33, 34]. It will be natural to use SCET to describe the collisional and radiative interactions of the jet in the medium when the typical transverse momentum of the partons in the jet is comparable to the size of the momentum exchange with the medium and much smaller than the jet energy. So far, only broadening of the final-state parton in semi-inclusive deeply inelastic scattering (SDIS) [35] has been considered in an effective theory of QCD. Reference [36] argues to present a less model-dependent result but its gauge invariance remains to be demonstrated explicitly.

To set up a general SCET framework that can describe the collisional and radiative processes induced by the propagation of an energetic parton in strongly interacting matter, to derive the operators that describe the momentum space evolution of the propagating quark or quark+gluon system and to demonstrate the gauge invariance of these results is the main goal of this paper. By comparing our findings to previous calculations of parton broadening [37, 38] and energy loss [39, 40, 41, 42] in nuclear matter we identify areas where the effective theory calculation will be able to improve the accuracy of existing computations.

Our manuscript is organized as follows: in section 2 we review very briefly the basic concepts of Soft Collinear Effective Theory. The kinematics of jet-medium interactions beyond the static scattering approximation is discussed in section 3. We identify the regime relevant to high energy jet production in hadronic reactions with large nuclei and elucidate the possibility for constructing an effective theory to describe parton propagation in dense QCD matter. The gauge-invariant Lagrangian for this effective theory is constructed in section 4 . Feynman rules are derived in the light-cone and covariant $R_{\xi}$ gauges. In

\footnotetext{
${ }^{1}$ We refer here to energy, momentum or mass scales.
} 
section 5 we evaluate the transverse momentum broadening of jets, induced by their collisional interactions in the strongly interacting medium. Radiative processes are discussed in section 6. Our focus here is on the soft gluon limit, when the energy of the emitted gluon $(\omega)$ is much less than the energy $(E)$ of the quark that splits: $\omega \ll E$, for comparison to previous results. We demonstrate the gauge invariance of the jet broadening and energy loss results in section 7. We deduce the kernels that describe the broadening and medium-induced bremsstrahlung as a function of the quark interactions in the medium in section 8 . An application of the reaction operators for collisional and radiative processes is also discussed in this section. The extension of radiative energy loss calculation beyond soft gluon approximation is presented in section 9. We also show how the process-dependent medium-induced radiative corrections factorize from the hard jet production cross section. Our conclusions are given in section 10. We have moved some of the background technical discussion to appendices.

\section{A brief overview of SCET}

SCET $[8,9,10,11]$ is an effective theory of QCD which describes the dynamics of highly energetic quarks and gluons. The relevant physical scales in this effective theory are the hard scale $E_{h} \sim E_{T} \sim E_{\mathrm{cm}}$, the jet scale $E_{j} \sim p_{\perp}$ that describes the width of the jet in momentum space and the scale of soft radiation $E_{s} \sim \Lambda_{\mathrm{QCD}}$. The power counting parameter of SCET $\lambda$ defines the hierarchy between the hard, jet and soft scales. We use the version of SCET, which is sometimes referred to as $\mathrm{SCET}_{\mathrm{I}}$, in which the scales are $E_{h} \sim \lambda^{0}, E_{j} \sim \lambda^{1}$ and $E_{s} \sim \lambda^{2}$. The degrees of freedom in SCET are collinear quarks $\left(\xi_{n, p}\right)$, collinear gluons $\left(A_{n, p}\right)$ and soft gluons $\left(A_{s}\right)$. Collinear particles have momentum in light-cone coordinates $p_{c} \sim\left[1, \lambda^{2}, \boldsymbol{\lambda}\right]$ and soft particles $p_{s} \sim\left[\lambda^{2}, \lambda^{2}, \lambda^{2}\right]$, where we define our light-cone notation in appendix A. All other fields, such as hard quarks and gluons, are integrated out from the QCD Lagrangian. Their effect on the dynamics is contained into the Wilson coefficients of the SCET operators, which can be calculated using a standard matching of full theory onto effective theory. In order to avoid confusion we note that what we call soft gluon mode in this paper $p_{s}^{\mu} \sim \lambda^{2}$ is sometimes called ultrasoft, while the soft momentum is defined as $p_{s}^{\mu} \sim \lambda$, see for example [11]. However, below in section 4 when we define the momentum scaling of the source, one of our choices corresponds to $p_{s}^{\mu} \sim \lambda$ and we call it a soft source.

The Lagrangian of SCET [9] arises from substituting into the QCD Lagrangian $\psi=\sum_{\tilde{p}} e^{-i \tilde{p} x} \psi_{n, \tilde{p}}$ and

integrating out the small component $\xi_{\bar{n}}$ of $\psi_{n}$, where $\xi_{n}=\frac{\eta \underline{n} \vec{n}}{4} \psi_{n}, \xi_{\bar{n}}=\frac{\vec{n} h \boldsymbol{h}}{4} \psi_{n}$ and $\psi_{n}=\xi_{n}+\xi_{\bar{n}}$. The result for the collinear-soft Lagrangian is:

$$
\begin{aligned}
& \mathcal{L}_{\mathrm{SCET}}\left(\xi_{n}, A_{n}, A_{s}\right)=\bar{\xi}_{n}\left[i n \cdot D+i \not D^{\perp} \frac{1}{i \bar{n} \cdot D} i \not D^{\perp}\right] \frac{\not h}{2} \xi_{n}+\mathcal{L}_{\mathrm{YM}}\left(A_{n}, A_{s}\right), \\
& \mathcal{L}_{\mathrm{YM}}\left(A_{n}, A_{s}\right)=\frac{1}{2 g^{2}} \operatorname{tr}\left\{\left[i D_{s}^{\mu}+g A_{n, q}^{\mu}, i D_{s}^{\nu}+g A_{n, q^{\prime}}^{\nu}\right]\right\}^{2}+\mathcal{L}_{\mathrm{G} . \mathrm{F} .}, \\
& \mathcal{L}_{\text {G.F. }}\left(R_{\xi}\right)=\frac{1}{\xi} \operatorname{tr}\left\{\left[i D_{s \mu}, A_{n, q}^{\mu}\right]\right\}^{2}, \\
& \mathcal{L}_{\mathrm{G} . \mathrm{F} .}(\mathrm{LCG}(b))=\frac{1}{\xi} \operatorname{tr}\left\{b_{\mu} A_{n, q}^{\mu}\right\}^{2} .
\end{aligned}
$$

Here, the covariant derivative $D$ contains both collinear and soft fields: $i D=i \partial+g\left(A_{n}+A_{s}\right)$, while $D_{s}$ includes only the soft gluons: $i D_{s}=i \partial+g A_{s}$. Thus, the collinear and soft modes are coupled in the SCET Lagrangian. In the first term of Eq. (2.2) the summation over label momenta $q, q^{\prime}$ is understood implicitly, and in Eq. (2.3), Eq. (2.4) summation over the label momentum $q$ is understood implicitly. We 
have written out explicitly the gauge fixing terms for the covariant and the light-cone gauges. The ghost terms are omitted for brevity.

A key ingredient of the SCET formulation is the BPS transformation [11]. This transformation constitutes a collinear field redefinition which involves soft Wilson lines and removes the interactions between soft and collinear fields in the Lagrangian of SCET up to the power corrections. Such decoupling is essential in the proof of factorization theorems in SCET. The BPS transformation redefines the collinear quark and gluon fields:

$$
\begin{array}{r}
\xi_{n, p}=Y \xi_{n, p}^{(0)}, \\
A^{a, \mu}=\mathcal{Y}^{a b} A_{n, p}^{(0) \mu, b},
\end{array}
$$

where the $Y(x)$ and $\mathcal{Y}^{a b}(x)$ are Wilson lines built out of the soft fields in the fundamental and adjoint representations correspondingly:

$$
\begin{aligned}
& Y(x)=\mathrm{P} \exp \left(i g \int_{-\infty}^{0} \mathrm{~d} s n \cdot A_{s}^{a}(n s+x) T^{a}\right), \\
& \mathcal{Y}^{a b}(x)=\mathrm{P} \exp \left(i g \int_{-\infty}^{0} \mathrm{~d} s n \cdot A_{s}^{e}(n s+x)\left(-i f^{e a b}\right)\right) .
\end{aligned}
$$

To derive the Lagrangian of SCET in terms of the decoupled collinear fields $\xi_{n}^{(0)}, A_{n}^{(0)}$ one needs the following key identities:

$$
\begin{aligned}
& Y T^{a} Y^{\dagger}=\mathcal{Y}^{b a} T^{b}, \\
& Y^{\dagger} n \cdot D Y=n \cdot D_{n} .
\end{aligned}
$$

In particular, the last equation removes the interactions between the soft gluons and the collinear quarks which is contained in the covariant derivative in the left hand side of the Eq. (2.10). As a result, after the BPS transformation we obtain:

$$
\mathcal{L}_{\mathrm{SCET}}\left(\xi_{n}, A_{n}, A_{s}\right)=\mathcal{L}_{c}\left(\xi_{n}, A_{n}\right)+\mathcal{L}_{s}\left(A_{s}\right)+\mathcal{L}_{c s}\left(\xi_{n}, A_{n}, A_{s}\right) \stackrel{\mathrm{BPS}}{\longrightarrow} \mathcal{L}_{c}\left(\xi_{n}^{(0)}, A_{n}^{(0)}\right)+\mathcal{L}_{s}\left(A_{s}\right) .
$$

The couplings between the collinear and soft modes is removed from the SCET Lagrangian. However, in order to preserve the gauge invariance one has to put soft and collinear Wilson lines into the external SCET operators. The collinear Wilson line is defined in the following way:

$$
W_{n}(x)=\mathrm{P} \exp \left(-i g \int_{0}^{\infty} \mathrm{d} s \bar{n} \cdot A_{n}^{a}(\bar{n} s+x) T^{a}\right) .
$$

\section{Kinematics of the in-medium jet interactions}

In this section we describe the kinematics of jet interactions in QCD matter. Our goal is to identify the typical momentum exchanges between the energetic incident partons and the medium, which in turn will help us construct an effective theory for these interactions.

The typical jet transverse energies that are phenomenologically interesting at RHIC are in the range $E_{T} \sim 10 \mathrm{GeV}-50 \mathrm{GeV}$. At the LHC this range is extended to $E_{T} \sim$ several hundred $\mathrm{GeV}$. On the other hand, the mass of the particles in the medium is on the order of or larger than $1 \mathrm{GeV}$. In cold nuclear 
matter this is the mass of the nucleon $m_{N}=0.94 \mathrm{GeV}$ and in the quark-gluon plasma this is the mass of dressed partons $\propto \mu=g T \sqrt{1+N_{f} / 6}$. Binding effects inside nuclear matter can significantly increase the effective mass of the recoiling particles. One of the main goals of this section is to study how the elastic scattering cross section depends on this mass and to identify the kinematic configurations that give a dominant contribution to this cross section.

The cross section for the elastic scattering of two particles of masses $m_{1}, m_{2}$ is given by the standard expression:

$$
d \sigma=\frac{1}{4 \sqrt{\left(p_{1} \cdot p_{2}\right)^{2}-m_{1}^{2} m_{2}^{2}}}\left\langle|M|^{2}\right\rangle \frac{d^{3} p_{3}}{(2 \pi)^{3} 2 E_{3}} \frac{d^{3} p_{4}}{(2 \pi)^{3} 2 E_{4}}(2 \pi)^{4} \delta^{4}\left(p_{1}+p_{2}-p_{3}-p_{4}\right) .
$$

We work in the rest frame of the medium, which for the cases of a quark-gluon plasma at mid-rapidity coincides with the laboratory frame. The same is true for fixed target $\mathrm{p}+\mathrm{A}$ experiments. In this frame the flux factor conveniently reduces to Flux $=4 m_{2} p_{1}$. Let us denote by $\theta$ the scattering angle of the incident parton $\left(\theta=\angle\left(p_{1}, p_{3}\right)\right)$ and by $p_{3}$ the physical solution arising from the energy constraint $E_{1}\left(p_{1}, m_{1}\right)+m_{2}=$ $E_{3}\left(p_{3}, m_{1}\right)+E_{4}\left(\vec{p}_{4}=\vec{p}_{1}-\vec{p}_{3}, m_{2}\right)$. In this paper we are interested in the case: $m_{1}<m_{2}$. In this case there is a single physical solution $p_{3}$. Substituting in Eq. (3.1), we obtain:

$$
\frac{d \sigma}{d \Omega}=\frac{1}{64 \pi^{2}} \frac{p_{3}^{2}}{p_{1} m_{2}\left[p_{3}\left(E_{1}+m_{2}\right)-p_{1} E_{3} \cos (\theta)\right]}\left\langle|M|^{2}\right\rangle .
$$

We now discuss the specific channels that may contribute to $\left\langle|M|^{2}\right\rangle$. To study jet broadening and energy loss, we will be interested in forward scattering where, on average, the direction of jet propagation is not significantly altered per interaction. Such scattering is dominated by the $t$-channel gluon exchange. One can, of course, write down $u$-channel and $s$-channel diagrams but these describe hard backward parton scattering and isotropisation processes rather than transverse momentum broadening and energy loss. For simplicity, we give the specific example of quark-quark scattering:

$$
\left\langle|M|^{2}\right\rangle=g^{4}\left(\frac{2}{9}\right)_{\text {color }} \frac{2\left(u^{2}+s^{2}\right)+4 m_{1}^{2}\left(2 t-2 m_{2}^{2}-m_{1}^{2}\right)+4 m_{2}^{2}\left(2 t-2 m_{1}^{2}-m_{2}^{2}\right)+8 m_{1}^{2} m_{2}^{2}}{t^{2}} .
$$

Here, one can conveniently take the $m_{1} \rightarrow 0, m_{2} \rightarrow 0$ limits. We note that if the interaction is of finite range, i.e. we have an exchange gluon of mass $m \sim \mu$, the only change is $t \rightarrow t-\mu^{2}$ in the denominator of Eq. (3.3).

We can now study the $m_{2}$ dependence of the average squared matrix element $\left\langle|M|^{2}\right\rangle$, the flux and phase space factors (PS, Flux), and the differential cross section $d \sigma / d \Omega$. We chose a massless incident quark of energy $10 \mathrm{GeV}$. The mass of the recoiling particle varies from $1 \mathrm{GeV}$ to $10^{3} \mathrm{GeV}$ and we use $g=2$ for the coupling constant. The top left and right panels in figure 1 show the differential phase space (normalized by the initial flux factor) and matrix element versus $\cos (\theta)$ with the leading $m_{2}^{2}$ dependencies taken out. While for $\cos (\theta)=1$ the curves come together, the difference for small values of $m_{2}$ away from the forward region can be many orders of magnitude. In contrast, in the differential cross section $d \sigma / d \Omega$ this variation largely cancels everywhere except for the backward scattering region. We show this result in the bottom panel of figure 1 . The insert illustrates the remaining subtle mass dependence in the forward scattering region. It is quite remarkable that this residual variation is less than $\pm 50 \%$. At the level of the integrated scattering cross section, the differences are even much smaller. For incident partons of $E=10 \mathrm{GeV}$ at RHIC and $E=100 \mathrm{GeV}$ at the LHC we obtain:

$$
\left.\frac{\sigma\left(m_{2}=1000 \mathrm{GeV}\right)-\sigma\left(m_{2}=1 \mathrm{GeV}\right)}{\sigma\left(m_{2}=1000 \mathrm{GeV}\right)}\right|_{\mathrm{RHIC}} \approx 13 \%,\left.\quad \frac{\sigma\left(m_{2}=1000 \mathrm{GeV}\right)-\sigma\left(m_{2}=1 G e V\right)}{\sigma\left(m_{2}=1000 \mathrm{GeV}\right)}\right|_{\mathrm{LHC}} \approx 2 \%
$$




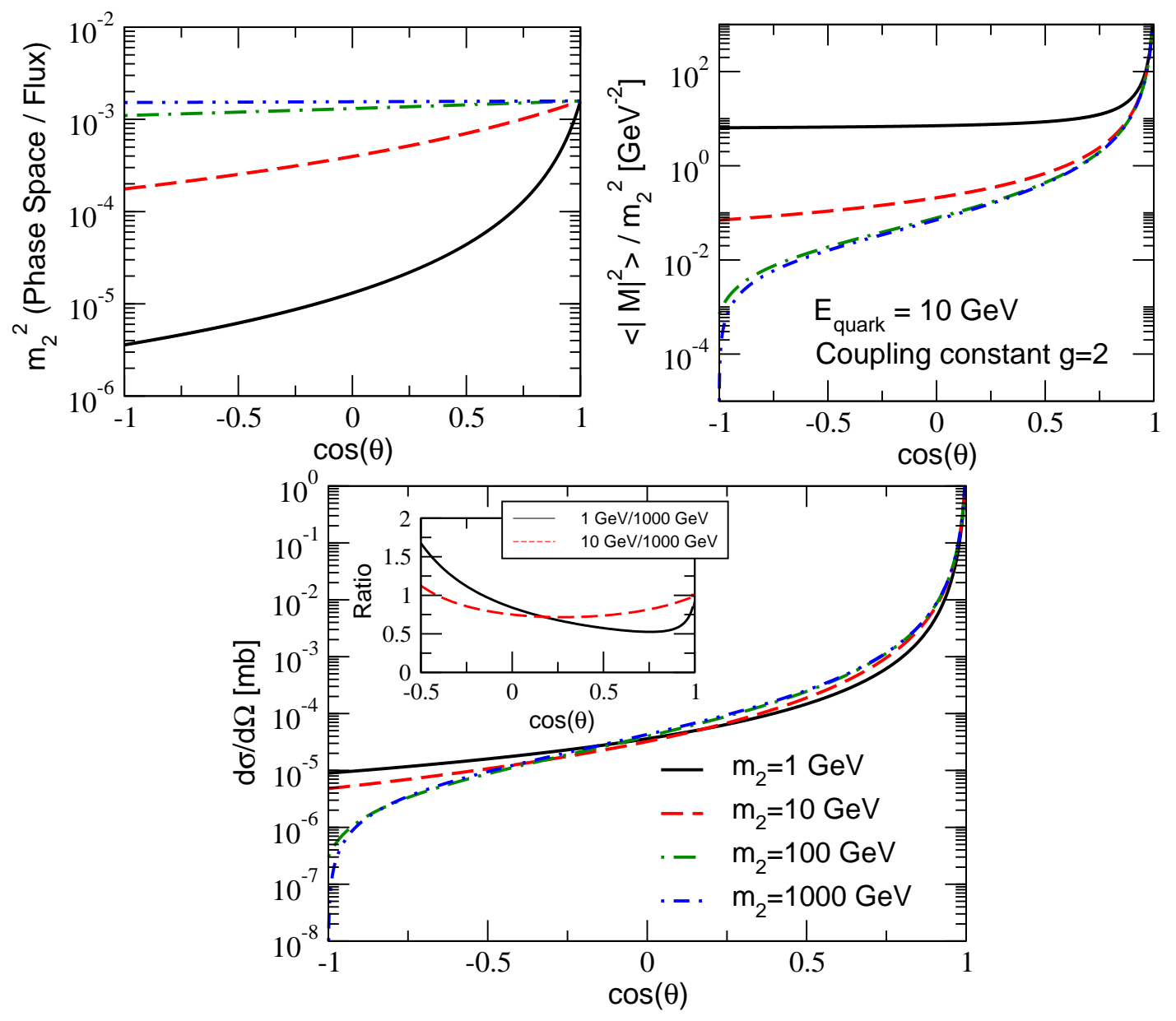

Figure 1: Kinematic factors and mean squared matrix element for the $t$-channel scattering of interest are shown versus $\cos (\theta)$ in the top left and top right panels, respectively. These exhibit strong dependence on the mass $m_{2}$ of the recoiling particle. The bottom panel shows the differential cross section where this $m_{2}$ dependence cancels over most of the $\theta$ range. The insert shows any residual differences as a ratio of $d \sigma / d \Omega$ for 2 different values of $m_{2}$.

respectively. Note that the scattering cross sections decreases for finite and small mass of the recoiling particle. This, in turn, leads to larger mean free paths $\lambda=1 / \sigma \rho$ in QCD matter and smaller radiative energy loss. This is in contrast to the result of Ref. [43]. The reason for this difference is that in [43] the general term "dynamical medium" was inaccurately used to describe a specific hard thermal loop approximation and the reported increase arises from the lack of magnetic screening. Lattice QCD results and non-perturbative arguments, however, suggest that magnetic screening effects may be present already at $\mathcal{O}\left(g^{2} T\right)$. Our results for a general finite-range interaction mediated by a massive vector particle allow to precisely quantify the effect of the medium recoil. For example, we found that more than $90 \%$ of the cross section comes from configurations where the jet is not deflected more than $15 \%$ from its original direction of propagation.

A small difference in the magnitude of the scattering cross section exists between the full calculation and the often used analytic approximation $\sigma=8 \pi \alpha_{s}^{2} / 9 \mu^{2}$ to quark-quark scattering. We can re-express 
the exact differential cross section as a function of the transverse momentum transfer as follows:

$$
\frac{d \sigma}{d \Omega} \rightarrow \frac{d \sigma}{d^{2} \mathbf{q}_{\perp}}=\frac{C_{2}(R) C_{2}(T)}{d_{A}} \frac{\left|v\left(\mathbf{q}_{\perp} ; E, m_{1}, m_{2}\right)\right|^{2}}{(2 \pi)^{2}} .
$$

In Eq. (3.4) $C_{2}(R), C_{2}(T)$ are the quadratic Casimirs for the incident and target parton representations, respectively, and $d_{A}=8$ is the dimension of the adjoint representation. The above expression also defines $v\left(\mathbf{q} ; E, m_{1}, m_{2}\right)$, which now depends on the jet energy and the masses of the scattering particles. It is easy to check that such a definition reduces to $v(\mathbf{q} ; E, 0, \infty)=\frac{4 \pi \alpha_{s}}{\boldsymbol{q}^{2}+\mu^{2}}$, consistent with similar definition in [40]. The only subtlety is that one allowed value of $\mathbf{q}_{\perp}$ generally corresponds to two values of $\cos (\theta)$. However, the region $-1<\cos (\theta)<0$ contributes $\sim 0.1 \%$ to the cross section and we simply ignore the second solution.

Finally we discuss which momentum region for the exchange gluon gives the dominant contribution to the cross section. The momentum transfer in terms of the final and initial jet momentum equals $q=p_{3}-p_{1}$. Writing this in laboratory frame in terms of the light-cone components, we get:

$$
q^{+}=E_{3}(1+\cos (\theta))-2 E_{1}, \quad q^{-}=E_{3}(1-\cos (\theta)), \quad\left|\boldsymbol{q}_{\perp}\right|=E_{3} \sin (\theta) .
$$

In the formula above we assumed that $m_{1}=0$ and $m_{2}$ is arbitrary. As we can clearly see from Figure 1 the cross section is dominated in the forward direction. Thus we assign to the leading region for the cross section the following power counting: $\theta \sim \lambda, E_{3}, E_{1} \sim 1$. We immediately can see from Eq. (3.5) that this power counting corresponds to the following leading momentum region, depending on the mass $m_{2}^{2}$ :

$$
\begin{aligned}
& q \sim\left[\lambda^{2}, \lambda^{2}, \boldsymbol{\lambda}\right], \text { if } m_{2} \sim \lambda^{0}, \text { i.e. static source, } \\
& q \sim\left[\lambda^{1}, \lambda^{2}, \boldsymbol{\lambda}\right], \text { if } m_{2} \sim \lambda^{1}, \text { i.e. soft source. }
\end{aligned}
$$

To summarize, we investigated in detail the kinematics of jet-medium interactions to determine the feasibility of an effective theory where these interactions are mediated by Glauber gluons. We showed that for the static source, which we use in sections 5-9 below, the cross section is indeed dominated by the Glauber momentum region. We also calculated the exact dependence of this scattering cross section on the energy and mass of the incident and recoiling particles. This cross section will be implemented to go beyond the static scattering center approximation. We note, however, that this approximation is remarkably good and within $15 \%$ of the exact result even for low energy jets.

\section{An effective theory for jet propagation in QCD matter: $\mathrm{SCET}_{\mathrm{G}}$}

As we have seen from the previous section, the effective theory of jet interactions in matter has to contain the Glauber mode, which carries the exchange momentum between the incident parton and the QCD medium with scaling $q \sim\left[\lambda^{2}, \lambda^{2}, \boldsymbol{\lambda}\right]$. Such mode is absent in SCET and we have to modify the theory by including it. One possibility was considered in [35] and later on used in [36] to study the multiple collisional interactions of jets. There are a few differences between our approach and these references. First of all, we write down the Glauber term directly in the momentum space as an effective potential [44], similar to the non-relativistic QCD (NRQCD) potential term [45]. Secondly, we consider a static source of Glauber

\footnotetext{
${ }^{2}$ Such sensitivity of $q^{+}$on the recoil mass arises from the fact that $E_{3}-E_{1}=m_{2}-\sqrt{m_{2}^{2}+\boldsymbol{q}^{2}}$, which is energy conservation equation. Thus, for heavy mass $m_{2} \sim 1$ the recoil energy is negligible $E_{3}-E_{1} \sim \lambda^{2}$ and for the lighter mass $m_{2} \sim \lambda$ it is comparable to the transverse momentum transfer: $E_{3}-E_{1} \sim \lambda$.
} 
gluons, whereas in [35] the source was a massless collinear field. We are motivated by the physical picture of nucleons or massive quasi-particles as sources of these Glauber gluons in nuclei and non-Abelian plasmas, respectively. We work in the rest frame of nuclear matter and also include the collinear gluons into the interaction Lagrangian with Glauber gluons.

In a different context, namely the Drell-Yan process, it has been shown that the Glauber mode has to be added to SCET for the consistency of the effective theory [46]. Having formulated a consistent effective theory $\mathrm{SCET}_{\mathrm{G}}$, it would be interesting to revisit the Drell-Yan factorization in this effective theory and understand the cancellation of Glauber gluons in inclusive Drell-Yan cross section from the effective theory point of view. In traditional QCD this cancellation was derived in Refs. [47, 48, 49], however it has not been addressed yet in effective theory methods to factorization.

\subsection{The $\mathrm{SCET}_{\mathrm{G}}$ Lagrangian for different sources and gauges}

Consider a quark or a gluon propagating in the positive light-cone direction $n$ in QCD matter. In this subsection we will derive the effective Lagrangian of $\mathrm{SCET}_{\mathrm{G}}$, which describes the interaction of our propagating jet with the source of the Glauber gluons. We consider three types of sources of Glauber gluons. The first one is a collinear field propagating in the $\bar{n}$ direction (considered in Refs [35], [36]). The second one is a (initially) static nucleon/nucleus or a massive quasi-particle. Interestingly, this source can be adequately described as a heavy quark effective theory (HQET) current. Finally, the third type of source that we consider is a soft parton of momentum $p \sim[\lambda, \lambda, \boldsymbol{\lambda}]$. For each of these three sources we consider three gauges: the $R_{\xi}$ gauge and two distinct light-cone gauges $A^{+}=0$ and $A^{-}=0$. In order to verify the symmetry properties of the Glauber interaction term we include the source fields into our Lagrangian. However, for practical calculations we integrate out the source fields as well as the Glauber gluons and present the resulting Feynman rules.

We start from the vector potential as a function of the QCD current of the source and the gluon propagator. Our method to derive the scaling for the vector potential $A_{G}^{\mu}(x)$ created by the Glauber gluons is same as in [35]:

$$
A_{G}^{\mu, a}(x)=\int d^{4} y \delta^{a b} D^{\mu \nu}(x-y) \bar{\psi}(y) g T^{b} \gamma_{\nu} \psi(y) .
$$

Expanding the propagator and the fermion field in the momentum space we get:

$$
A_{G}^{\mu, a}(x)=\int \frac{d^{3} \boldsymbol{p}}{\sqrt{2 E_{\boldsymbol{p}}}(2 \pi)^{3}} \frac{d^{3} \boldsymbol{q}}{\sqrt{2 E_{\boldsymbol{p}+\boldsymbol{q}}}(2 \pi)^{3}} \sum_{s, r} a_{\boldsymbol{p}+\boldsymbol{q}}^{r \dagger} a_{\boldsymbol{p}}^{s} \bar{u}(\boldsymbol{p}+\boldsymbol{q}, r) g T^{a} \gamma_{\nu} u(\boldsymbol{p}, s) \mathrm{e}^{-i q x}(-i) \Delta^{\mu \nu}(q)+\cdots,\left(4^{2}\right.
$$

where we have written down only the part of the vector potential that contributes to particle-particle scattering, and the three remaining combinations involving anti-particles are omitted. In each of three gauges under consideration, the gluon propagator is equal to:

$$
\begin{gathered}
\text { Covariant gauge }\left[\Delta^{\mu \nu}(q)\right]_{R_{\xi}}=\frac{\left(g^{\mu \nu}-\frac{q^{\mu} q^{\nu}}{\mu^{2}}(1-\xi)\right)}{q^{2}-\mu^{2}} \rightarrow \frac{g^{\mu \nu}}{q^{2}-\mu^{2}}, \\
\text { Light-cone }\left(A^{+}=0\right) \text { gauge }\left[\Delta^{\mu \nu}(q)\right]_{A^{+}}=\frac{\left(g^{\mu \nu}-\frac{\bar{n}^{\mu} q^{\nu}+\bar{n}^{\nu} q^{\mu}}{q^{+}}\right)}{q^{2}-\mu^{2}} \rightarrow \frac{g^{\mu \nu}-\frac{\bar{n}^{\nu} q^{\mu}}{q^{+}}}{q^{2}-\mu^{2}}, \\
\text { Light-cone }\left(A^{-}=0\right) \text { gauge }\left[\Delta^{\mu \nu}(q)\right]_{A^{-}}=\frac{\left(g^{\mu \nu}-\frac{n^{\mu} q^{\nu}+n^{\nu} q^{\mu}}{q^{-}}\right)}{q^{2}-\mu^{2}} \rightarrow \frac{g^{\mu \nu}-\frac{n^{\nu} q^{\mu}}{q^{-}}}{q^{2}-\mu^{2}},
\end{gathered}
$$




\begin{tabular}{|c|c||c||c||c||}
\hline Gauge & Object & Collinear source & Static source & Soft source \\
\hline \hline & $p$ & {$\left[\lambda^{2}, 1, \boldsymbol{\lambda}\right]$} & {$[1,1, \boldsymbol{\lambda}]$} & {$[\lambda, \lambda, \boldsymbol{\lambda}]$} \\
& $a_{\boldsymbol{p}}, a_{\boldsymbol{p}}^{\dagger}$ & $\lambda^{-1}$ & $\lambda^{-3 / 2}$ & $\lambda^{-3 / 2}$ \\
& $u(p)$ & 1 & 1 & $\lambda^{1 / 2}$ \\
& $\bar{u}\left(p_{2}\right) \gamma_{\nu} u\left(p_{1}\right)$ & {$\left[\lambda^{2}, 1, \boldsymbol{\lambda}\right]$} & {$[1,1, \boldsymbol{\lambda}]$} & {$[\lambda, \lambda, \boldsymbol{\lambda}]$} \\
\hline \hline$R_{\xi}$ & $A^{\mu}(x)$ & {$\left[\lambda^{4}, \lambda^{2}, \boldsymbol{\lambda}^{3}\right]$} & {$\left[\lambda^{2}, \lambda^{2}, \boldsymbol{\lambda}^{3}\right]$} & {$[\lambda, \lambda, \boldsymbol{\lambda}]$} \\
& $\Gamma_{\mathrm{qqA}_{\mathrm{G}}}$ & $\Gamma_{1}^{\mu}$ & $\Gamma_{1}^{\mu}$ & $\Gamma_{1}^{\mu}$ \\
& $\Gamma_{\mathrm{ggA}_{\mathrm{G}}}$ & $\Sigma_{1}^{\mu \nu \lambda}$ & $\Sigma_{1}^{\mu \nu \lambda}$ & $\Sigma_{1}^{\mu \nu \lambda}$ \\
& $\Gamma_{\mathrm{s}}$ & $\Gamma_{1}^{\mu}(n \leftrightarrow \bar{n})$ & $\Gamma_{3}^{\mu}$ & $\Gamma_{4}^{\mu}$ \\
\hline \hline$A^{+}=0$ & $A^{\mu}(x)$ & {$\left[0, \lambda^{2}, \boldsymbol{\lambda}^{3}\right]$} & {$\left[0, \lambda^{2}, \boldsymbol{\lambda}\right]$} & {$[0, \lambda, \mathbf{1}]$} \\
& $\Gamma_{\mathrm{qqA}}$ & $\Gamma_{1}^{\mu}$ & $\Gamma_{1}^{\mu}+\Gamma_{2}^{\mu}$ & $\Gamma_{1}^{\mu}+\Gamma_{2}^{\mu}$ \\
& $\Gamma_{\mathrm{ggA}_{\mathrm{G}}}$ & $\Sigma_{2}^{\mu \nu \lambda}$ & $\Sigma_{2}^{\mu \nu \lambda}$ & $\Sigma_{2}^{\mu \nu \lambda}$ \\
& $\Gamma_{\mathrm{s}}$ & $\Gamma_{2}^{\mu}(n \leftrightarrow \bar{n})$ & $\Gamma_{3}^{\mu}$ & $\Gamma_{4}^{\mu}$ \\
\hline \hline$A^{-}=0$ & $A^{\mu}(x)$ & {$\left[\lambda^{2}, 0, \boldsymbol{\lambda}\right]$} & {$\left[\lambda^{2}, 0, \boldsymbol{\lambda}\right]$} & {$[\lambda, 0, \mathbf{1}]$} \\
& $\Gamma_{\mathrm{qqA}}$ & $\Gamma_{2}^{\mu}$ & $\Gamma_{2}^{\mu}$ & $\Gamma_{2}^{\mu}$ \\
& $\Gamma_{\mathrm{ggA}_{\mathrm{G}}}$ & $\Sigma_{3}^{\mu \nu \lambda}$ & $\Sigma_{3}^{\mu \nu \lambda}$ & $\Sigma_{3}^{\mu \nu \lambda}$ \\
& $\Gamma_{\mathrm{s}}$ & $\Gamma_{1}^{\mu}(n \leftrightarrow \bar{n})$ & $\Gamma_{3}^{\mu}$ & $\Gamma_{4}^{\mu}$ \\
\hline
\end{tabular}

Table 1: Summary of the scaling behavior of the Glauber gluon source ingredients, the Glauber vector potential and the Feynman rules for the newly constructed theory $\mathrm{SCET}_{\mathrm{G}}$ in the covariant $R_{\xi}$ gauge and two different light-cone gauges $A^{+}=0, A^{-}=0$.

where the arrow in each line indicates that the term proportional to $q_{\nu}$ vanishes in Eq. (4.2) because the external source particles are on-shell: $q^{\nu} \bar{u}(p+q) \gamma_{\nu} u(p)=0$. In Eqs. (4.3) - (4.5) we also use a finite range interaction mediated by a vector field of mass $\mu$.

Using Eq. (4.2) and Eqs. (4.3) - (4.5) we obtain the scaling formula for $A_{G}^{\mu}(x)$ for all cases. The scaling of the creation operators is derived from the anti-commutation relations in momentum space by allowing the external momentum of the source particles in each of three cases under consideration to be correspondingly: collinear, soft $^{3}$ and soft. We also note that the vector $p$ is collinear, while $q$ has Glauber scaling for collinear and static sources, and $q$ has scaling given by Eq. (3.7) for the soft source ${ }^{4}$. As a result we complete in table 4.1 all entries for the scaling of $A_{G}^{\mu}(x)$.

Having determined the scaling of the vector potential created by the Glauber field for the cases of interest, we now derive the effective theory Feynman rules for the coupling of the energetic jet to the Glauber field. We read off the Feynman rules from the usual SCET Lagrangian Eqs. (2.1) - (2.4) by treating the vector potential created by Glauber gluons in the covariant derivative as a background field. All Feynman graphs between collinear and Glauber gluons contained in Eq. $(2.2)^{5}$ can be found using derived Feynman rules in the background field method from Ref. [50]. In addition to these rules, one has

\footnotetext{
${ }^{3}$ For the static source the external momentum equals $p=m v+k$, however $p_{1}-p_{2}=k_{1}-k_{2}$, equals the difference of two soft momenta.

${ }^{4}$ Throughout this section we refer to both scalings in Eq. (3.6) and Eq. (3.7) as Glauber gluons, however it should be clear from the context which one is used when.

${ }^{5}$ Those rules include couplings of a Glauber gluon to two and three collinear gluons, and two Glaubers with two collinear gluons. We present in the table only the first vertex $\Gamma_{\mathrm{ggA}}$.
} 
to apply the specific power counting of the vector potential, which we derived for each source and gauge. As a result, we fill in the table the vertices $\Gamma_{\mathrm{qqA}_{\mathrm{G}}}, \Gamma_{\mathrm{ggA}}$ for each source and for each gauge. Finally, we include in table 4.1 the Feynman rules $\Gamma_{\mathrm{s}}$ for the interaction between the source fields and the Glauber gluons. This is achieved by noting that we can view the jet moving in the direction of $n$ as a source of Glauber gluons for the target fields $\bar{\eta}, \eta$. Thus, the Feynman rules $\Gamma_{s}$ can be found by using our scaling rules for $A_{G}^{\mu}(x)$ with the collinear source in the $n$-direction ${ }^{6}$. Also, note that for the collinear source we use SCET current in the $\bar{n}$ direction, for the static source we use the HQET current and, finally, for the soft source we use the unexpanded vertex $\gamma_{\mu}$ consistent with the soft quark interaction with the background field. As a result we fill in all the elements of table 4.1, where we have defined $\Gamma_{1} \ldots \Gamma_{4}, \Sigma_{1} \ldots \Sigma_{3}$ as follows:

$$
\begin{aligned}
& \Gamma_{1}^{\mu, a}=i g T^{a} n^{\mu} \frac{\not h}{2} \\
& \Gamma_{2}^{\mu, a}=i g T^{a} \frac{\gamma_{\perp}^{\mu} \not p_{\perp}+\not p_{\perp}^{\prime} \gamma_{\perp}^{\mu}}{\bar{n} \cdot p} \frac{\not h}{2}, \\
& \Gamma_{3}^{\mu, a}=i g T^{a} v^{\mu} \\
& \Gamma_{4}^{\mu, a}=i g T^{a} \gamma^{\mu} \\
& \Sigma_{1}^{\mu \nu \lambda, a b c}=g f^{a b c} n^{\mu}\left[g^{\nu \lambda} \bar{n} \cdot p+\bar{n}^{\nu}\left(p_{\perp}^{\prime \lambda}-p_{\perp}^{\lambda}\right)-\bar{n}^{\lambda}\left(p_{\perp}^{\prime \nu}-p_{\perp}^{\nu}\right)-\frac{1-\frac{1}{\xi}}{2}\left(\bar{n}^{\lambda} p^{\nu}+\bar{n}^{\nu} p^{\prime \lambda}\right)\right], \\
& \Sigma_{2}^{\mu \nu \lambda, a b c}=g f^{a b c}\left[g_{\perp}^{\mu \lambda}\left(-\frac{n^{\nu}}{2} p^{+}+p_{\perp}^{\nu}-2 p_{\perp}^{\prime \nu}\right)+g_{\perp}^{\mu \nu}\left(-\frac{n^{\lambda}}{2} p^{+}+p_{\perp}^{\prime \lambda}-2 p_{\perp}^{\lambda}\right)\right. \\
& \left.+g_{\perp}^{\nu \lambda}\left(n^{\mu} \bar{n} \cdot p+p_{\perp}^{\mu}+p_{\perp}^{\prime \mu}\right)\right] \\
& \Sigma_{3}^{\mu \nu \lambda, a b c}=g f^{a b c}\left[g_{\perp}^{\mu \lambda}\left(\frac{\bar{n}^{\nu}}{2}\left(p^{-}-2 p^{\prime-}\right)+p_{\perp}^{\nu}-2 p_{\perp}^{\prime \nu}\right)+g_{\perp}^{\mu \nu}\left(\frac{\bar{n}^{\lambda}}{2}\left(p^{\prime-}-2 p^{-}\right)+p_{\perp}^{\prime \lambda}-2 p_{\perp}^{\lambda}\right)\right. \\
& \left.+g_{\perp}^{\nu \lambda}\left(p_{\perp}^{\mu}+p_{\perp}^{\prime \mu}\right)\right] .
\end{aligned}
$$

The derived rules allow us to write down the effective Lagrangian of $\mathrm{SCET}_{\mathrm{G}}$. As a result, we obtain the following interaction term between SCET collinear fields and the vector potential $A_{G}^{\mu}(x)$ of the Glauber gluons:

$$
\begin{aligned}
& \mathcal{L}_{\mathrm{SCET}_{\mathrm{G}}}\left(\xi_{n}, A_{n}, A_{G}\right)=\mathcal{L}_{\mathrm{SCET}}\left(\xi_{n}, A_{n}\right)+\mathcal{L}_{\mathrm{G}}\left(\xi_{n}, A_{n}, A_{G}\right), \\
& \mathcal{L}_{\mathrm{G}}\left(\xi_{n}, A_{n}, A_{G}\right)=\sum_{p, p^{\prime}} \mathrm{e}^{-i\left(p-p^{\prime}\right) x}\left(\bar{\xi}_{n, p^{\prime}} \Gamma_{\mathrm{qqA}_{\mathrm{G}}}^{\mu, a} \frac{\not h}{2} \xi_{n, p}-i \Gamma_{\mathrm{ggA}_{\mathrm{G}}}^{\mu \nu \lambda, a b c}\left(A_{n, p^{\prime}}^{c}\right)_{\lambda}\left(A_{n, p}^{b}\right)_{\nu}\right) A_{\mathrm{G} \mu, a}(x) .
\end{aligned}
$$

Depending on the gauge and the source, the vertices and the vector potential are different and are provided in the table above. The Lagrangian of this form for the collinear source in $R_{\xi}$ and $A^{-}=0^{7}$ gauges was derived in [35] and agrees with our expressions for corresponding two entries for $\Gamma_{\mathrm{qqA}_{\mathrm{G}}}$ in table 4.1. We

\footnotetext{
${ }^{6}$ Note that in the table we have derived the Feynman rules generated by the collinear source moving in the $\bar{n}$ direction. However, since our target is a collinear current in the $n$-direction, its effect on the source can be derived from our table by reversing the $n \leftrightarrow \bar{n}$ in the collinear source column.

${ }^{7}$ In order to avoid confusion we note that in [35] the source was in the $n$ direction while the target jet in the $\bar{n}$ direction, thus our formulas agree with that reference if $n \leftrightarrow \bar{n}$ as expected. For example in [35] the light-cone gauge $A^{+}=0$ was considered, while it is analogous to our $A^{-}=0$ gauge.
} 
also note that for the covariant gauge and $\xi=1$ our Feynman rule for $\Gamma_{g_{g A}}\left(R_{\xi}\right)=\Sigma_{1}^{\mu \nu \lambda}$ disagrees with that of [36]. The corresponding Feynman rule from Ref. [36] contains only the first term in Eq. (4.10). However, note that with such a Feynman rule, the relation Eq. (7.4) below would be violated, which would lead to different results for single Born radiative loss (see section 6) calculated in the covariant and the hybrid gauges ${ }^{8}$. With our Feynman rule this inconsistency does not happen.

Finally, in order to analyze the invariance of our Glauber exchange terms for quarks and gluons under the gauge symmetries of SCET, we rewrite Eq. (4.14) including the source fields (see Eq. (4.1)):

$$
\mathcal{L}_{\mathrm{G}}\left(\xi_{n}, A_{n}, \eta\right)=\sum_{p, p^{\prime}, q} \mathrm{e}^{-i\left(p-p^{\prime}+q\right) x}\left(\bar{\xi}_{n, p^{\prime}} \Gamma_{\mathrm{qqA}_{\mathrm{G}}}^{\mu, a} \frac{\not h}{2} \xi_{n, p}-i \Gamma_{\mathrm{ggA}_{\mathrm{G}}}^{\mu \nu \lambda, a b c}\left(A_{n, p^{\prime}}^{c}\right)_{\lambda}\left(A_{n, p}^{b}\right)_{\nu}\right) \bar{\eta} \Gamma_{\mathrm{s}}^{\delta, a} \eta \Delta_{\mu \delta}(q),
$$

where all the vertices for the target and the source are provided conveniently in table 4.1. In order to make this Lagrangian collinear gauge invariant one needs to dress the quarks and gluons with collinear Wilson lines $W_{n}(x)$, defined in Eq. (2.12). As a result the Lagrangian that includes the Wilson lines can be obtained as follows:

$$
\mathcal{L}_{\mathrm{G}}\left(\xi_{n}, A_{n}, \eta\right) \rightarrow \mathcal{L}_{\mathrm{G}}\left(W_{n}^{\dagger} \xi_{n}, \mathcal{B}_{n}\left(A_{n}\right), \eta\right) \equiv \mathcal{L}_{\mathrm{G}}\left(\chi_{n}, \mathcal{B}_{n}, \eta\right)
$$

where $W_{n}^{\dagger} \xi_{n}\left(\equiv \chi_{n}\right), \mathcal{B}_{n}\left(A_{n}\right)$ are the dressed collinear gauge invariant quark and gluon fields, correspondingly. In the next subsection we will show that Lagrangian in Eq. (4.16) is invariant under the soft and collinear gauge transformations of SCET.

The derived Lagrangian of $\mathrm{SCET}_{\mathrm{G}}$ in Eq. (4.14) and Eq. (4.15) contains only interaction between a single collinear quark or gluon with a single Glauber gluon. However there are additional interactions between the collinear particles and Glauber gluons. For example, in the light-cone gauge the first term of Eq. (2.1) contains an interaction where the two Glauber gluons interact at the same point with a collinear quark line. The same Lagrangian in the same gauge contains the interaction where at the same point there are a collinear quark, a collinear gluon and a Glauber gluon. While we omit these terms in this section for brevity, their derivation is straightforward and the corresponding Feynman rules are listed in the appendix $\mathrm{C}$.

While we derived the Feynman rules of $\mathrm{SCET}_{\mathrm{G}}$ for variety of sources and gauges, in the main part of this paper we will do calculations in the following cases. For the source, motivated by our study in section 3, which showed that at RHIC and LHC energies the recoil effect is negligible with accuracy better than $15 \%$, we will use the (initially) static source. The interested reader will notice that since the physics results depend on the transverse momentum exchanges (Glauber gluons) between the projectile and the target and the jet-medium cross sections, they should not be sensitive to the components of the source in the $n$ and $\bar{n}$ directions.

As for the gauge choice, we consider three cases in this paper, which allows us to establish the gauge invariance of the broadening and the radiative energy loss results. The first two choices are the covariant and positive light-cone gauges. All relevant Feynman rules are derived in this section and summarized in appendix C. Note that in both cases the Glauber gluons and collinear gluons are quantized with the same gauge-fixing condition. However there is no problem in quantizing the collinear gluons in, say, the light-cone gauge, while the Glauber gluons are in the covariant gauge. This is our third choice and we call it the hybrid gauge. For practical purposes this turns out to be the most convenient gauge choice [40, 51].

\footnotetext{
${ }^{8}$ This gauge choice is defined at the end of this section.
} 
Physically, such a choice is possible since the scattering part and the medium-induced splitting parts of the calculation factorize. From a formal point of view the hybrid gauge corresponds to the vector potential created by the source derived in the covariant gauge, and the SCET Lagrangian quantized in the light-cone gauge with the corresponding background field vector potential. We discuss in details the Feynman rules in this gauge in section 7.1 and in appendix $\mathrm{C}$.

\subsection{Gauge invariance of $\mathcal{L}_{\mathrm{G}}$}

In this subsection we show that the Glauber Lagrangian $\mathcal{L}_{\mathrm{G}}$ is invariant under both collinear and soft gauge transformations in SCET. The collinear gauge symmetry is a simple consequence of dressing the collinear fields with the collinear Wilson lines. The fields transform under the collinear gauge transformation of SCET according to [11]:

$$
\begin{array}{ll}
\xi_{n} \rightarrow \mathcal{U}_{c} \xi_{n}, & \chi_{n} \equiv W_{n}^{\dagger} \xi_{n} \rightarrow \chi_{n}, \\
A_{n}^{\mu} \rightarrow \mathcal{U}_{c} A_{n}^{\mu} \mathcal{U}_{c}^{\dagger}+\frac{1}{g} \mathcal{U}_{c} i D^{\mu} \mathcal{U}_{c}^{\dagger}, & \mathcal{B}_{n}^{\mu} \equiv \frac{1}{g}\left[W_{n}^{\dagger} i D^{\mu} W_{n}\right] \rightarrow \mathcal{B}_{n}^{\mu}, \\
h_{v} \rightarrow h_{v}, &
\end{array}
$$

where $W$ is the collinear Wilson line, which is defined in Eq. (2.12), and the square brackets in the last equation indicate that the derivative operator acts only within the brackets. Note that the massive fields $h_{v}$ do not transform under the collinear transformation, since $p_{h} \sim[1,1, \boldsymbol{\lambda}]$ and $p_{c} \sim\left[1, \lambda^{2}, \boldsymbol{\lambda}\right]$. As we see from the definition of $\mathcal{L}_{G}\left(\chi, \mathcal{B}, h_{v}\right)$, since it is explicitly built out of the gauge invariant collinear fields, the Lagrangian is invariant under collinear gauge transformation:

$$
\mathcal{L}_{G}\left(\chi, \mathcal{B}, h_{v}\right) \stackrel{\text { collinear gauge transformation }}{\longrightarrow} \mathcal{L}_{G}\left(\chi, \mathcal{B}, h_{v}\right) .
$$

Demonstrating the invariance of the Lagrangian of $\mathrm{SCET}_{\mathrm{G}}$ under the soft gauge transformation is slightly more involved. The soft transformation of SCET looks like [11]:

$$
\begin{array}{ll}
\xi_{n} \rightarrow V_{s} \xi_{n}, & \chi_{n} \equiv W_{n}^{\dagger} \xi_{n} \rightarrow V_{s} \chi_{n}, \\
A_{n}^{\mu} \rightarrow V_{s} A_{n}^{\mu} V_{s}^{\dagger}, & \mathcal{B}_{n}^{\mu} \equiv \frac{1}{g}\left[W_{n}^{\dagger} i D^{\mu} W_{n}\right] \rightarrow V_{s} \mathcal{B}_{n}^{\mu} V_{s}^{\dagger}, \\
h_{v} \rightarrow V_{s} h_{v}, &
\end{array}
$$

where $V_{s}=\mathrm{e}^{i \alpha^{a}(x) T^{a}}$, such that $\partial_{\mu} V_{s}(x)=\mathcal{O}\left(\lambda^{2}\right)$. For shorthand notation, define $Y \equiv V_{s}$. The quark part of the Lagrangian of $\mathrm{SCET}_{\mathrm{G}}$ then transforms into:

$$
\begin{aligned}
& T_{i j}^{c} \otimes T_{k l}^{c} \stackrel{\text { soft gauge transformation }}{\longrightarrow}\left(Y^{\dagger} T^{c} Y\right)_{i j} \otimes\left(Y^{\dagger} T^{c} Y\right)_{k l}=\left(Y_{i m}^{\dagger} T_{m n}^{c} Y_{n j}\right) \otimes\left(Y_{k f}^{\dagger} T_{f g}^{c} Y_{g l}\right) \\
& =\left(Y_{i m}^{\dagger} Y_{n j}\right)\left(Y_{k f}^{\dagger} Y_{g l}\right)\left(\frac{1}{2} \delta_{m g} \delta_{n f}-\frac{1}{2 N} \delta_{m n} \delta_{f g}\right)=\frac{1}{2} \delta_{i l} \delta_{j k}-\frac{1}{2 N} \delta_{i j} \delta_{k l}=T_{i j}^{c} \otimes T_{k l}^{c} .
\end{aligned}
$$

Similarly, for the gluon part of the $\mathcal{L}_{G}$ in Eq. (4.16) we have:

$$
\begin{aligned}
& A^{\mu, a} A^{\nu, b} i f^{a b c} \otimes T^{c} \equiv A^{\mu, a} A^{\nu, b} \otimes\left[T^{a}, T^{b}\right] \stackrel{\text { soft gauge transformation }}{\longrightarrow} \mathcal{Y}^{a a^{\prime}} A^{\mu, a^{\prime}} \mathcal{Y}^{b b^{\prime}} A^{\nu, b^{\prime}} \otimes Y^{\dagger}\left[T^{a^{\prime}}, T^{b^{\prime}}\right] Y \\
& =A^{\mu, a^{\prime}} A^{\nu, b^{\prime}} \otimes Y^{\dagger}\left[\mathcal{Y}^{a a^{\prime}} T^{a^{\prime}}, \mathcal{Y}^{b b^{\prime}} T^{b^{\prime}}\right] Y=A^{\mu, a^{\prime}} A^{\nu, b^{\prime}} \otimes Y^{\dagger}\left[Y T^{a^{\prime}} Y^{\dagger}, Y T^{b^{\prime}} Y^{\dagger}\right] Y \\
& =A^{\mu, a^{\prime}} A^{\nu, b^{\prime}} \otimes\left[T^{a^{\prime}}, T^{b^{\prime}}\right]=A^{\mu, a^{\prime}} A^{\nu, b^{\prime}} i f^{a^{\prime} b^{\prime} c^{\prime}} \otimes T^{c^{\prime}}
\end{aligned}
$$

As a result, we obtain that the Glauber term in $\mathrm{SCET}_{\mathrm{G}}$ is invariant under the soft gauge transformation:

$$
\mathcal{L}_{G}\left(\chi, \mathcal{B}, h_{v}\right) \stackrel{\text { soft gauge transformation }}{\longrightarrow} \mathcal{L}_{G}\left(\chi, \mathcal{B}, h_{v}\right) .
$$




\section{Jet broadening}

In this section we derive the modification to the transverse momentum distribution of jets from an elastic in-medium scattering to first order in opacity. We consider a quark jet or a gluon jet interacting with an initially static fermionic center for definitiveness. The necessary effective theory SCET $_{\mathrm{G}}$ Feynman rules were derived in the section 4 and we use the covariant gauge for our calculation. The amplitudes that we consider for the quark and gluon cases have the following form, respectively:

$$
\begin{aligned}
& A^{(\mathrm{q})}=\left\langle J\left|T \bar{\chi}_{n}\left(x_{0}\right) \mathrm{e}^{i \int d^{4} x\left(\mathcal{L}^{\mathrm{QCD}}+\mathcal{L}^{\mathrm{SCET}_{\mathrm{G}}}\right)}\right| \mathbf{p}\right\rangle, \\
& A^{(\mathrm{g})}=\left\langle J_{\mu, a}\left|T \mathcal{B}_{n}^{\mu, a}\left(x_{0}\right) \mathrm{e}^{i \int d^{4} x\left(\mathcal{L}^{\mathrm{QCD}}+\mathcal{L}^{\mathrm{SCET}_{\mathrm{G}}}\right)}\right| \mathbf{p}\right\rangle,
\end{aligned}
$$

where $\chi_{n}$ and $\mathcal{B}_{n}$ are the gauge invariant quark and Gluon fields in SCET, and $J$ is the underlying hard process that creates the quark or gluon jet. The $\mathcal{L}^{\mathrm{QCD}}$ term simply generates the hard QCD process in question, which we take into account by effective Feynman rule $\left\langle J\left|\bar{\chi}_{n}\left(x_{0}\right)\right| \mathbf{p}\right\rangle=\bar{\chi}_{n, p} i J(p) \mathrm{e}^{i p x_{0}}$. In the next two subsections we calculate these amplitudes using the Lagrangian of SCET $_{\mathrm{G}}$ and combining the single Glauber gluon exchange diagram with the contact limit of the two Glauber gluon exchange diagrams. The corresponding amplitudes are called single and double Born diagrams in the literature [37, 38]. Each interaction in medium can be considered located at a certain point $\boldsymbol{x}$, and an integral over the Glauber gluon momentum is introduced. To keep formulas compact, it is convenient to use the following shorthand notation:

$$
\begin{aligned}
& d \Phi_{i}=\frac{d^{4} q_{i}}{(2 \pi)^{4}} \mathrm{e}^{i q_{i} \delta x_{i}} v\left(q_{i}\right), \\
& d \boldsymbol{\Phi}_{i \perp}=\frac{d^{2} \boldsymbol{q}_{i \perp}}{(2 \pi)^{2}} \mathrm{e}^{-i \boldsymbol{q}_{i \perp} \delta \boldsymbol{x}_{i \perp}} \tilde{v}\left(\boldsymbol{q}_{i \perp}\right),
\end{aligned}
$$

where $i$ is the index of the corresponding scattering center, $v(q)=2 \pi \delta\left(q^{0}\right) \tilde{v}\left(\boldsymbol{q}_{\perp}\right), \tilde{v}\left(\boldsymbol{q}_{\perp}\right)$ is defined by Eq. (3.4) and $\delta x_{i}=x_{i}-x_{0}$. The delta function in $v(q)$ makes the $d q^{+}$integral trivial, while the $q^{-}$integral still needs to be evaluated. The following identity arises once one works out the $d^{4} q$ in the light-cone coordinates:

$$
d \Phi_{i}=d \Phi_{i \perp} \frac{d q_{i}^{-}}{2 \pi} \mathrm{e}^{i q^{-} \delta z_{i}}
$$

where $\delta z_{i}=\delta \boldsymbol{x}_{i}^{3}$. To also shorten the color notation we substitute the color generator $T^{a}$ by simply $a$ and specifying the representation it belongs to. For example:

$$
\begin{aligned}
& (a)_{R}(a)_{T_{i}}=T^{a}(R) T^{a}(i), \\
& {[a, b]_{R}=i f^{a b c} T^{c}(R) .}
\end{aligned}
$$

We use $R$ to denote the color representation of the incident energetic quark or gluon, and we use $T$ to specify the color representation of the scattering center itself. For example, $d_{T}=3$ since we consider a fermionic scattering center, while $d_{R}=d_{F}=3$ for a quark jet and $d_{R}=d_{A}=8$ for a gluon jet.

For the quark (or gluon) propagator with the collinear plus Glauber momentum $p-q$, where $p$ has scaling $\left[1, \lambda^{2}, \boldsymbol{\lambda}\right]$ and $q$ is the Glauber gluon with the momentum scaling $\left[\lambda^{2}, \lambda^{2}, \boldsymbol{\lambda}\right]$, we define:

$$
\Delta_{g}(p, q) \equiv \frac{1}{p^{-}-q^{-}-\frac{\left(p_{\perp}-q_{\perp}\right)^{2}-i \varepsilon}{p^{+}}} .
$$




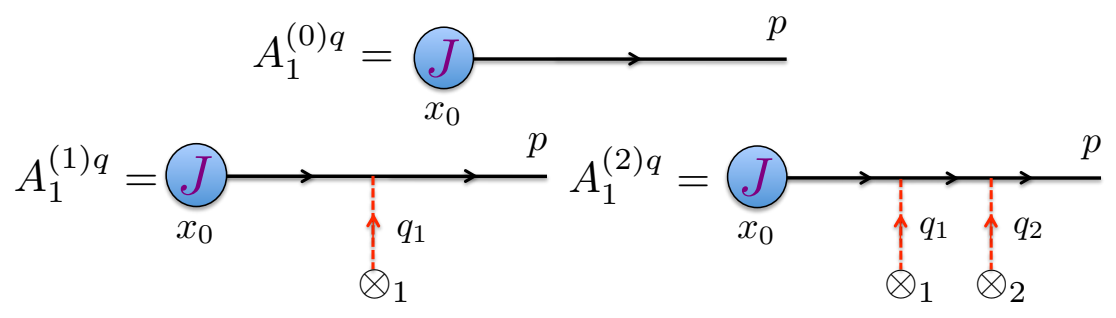

Figure 2: Three lowest order diagrams contributing to a collisional in-medium quark interaction. Top: tree level, bottom left: single Born diagram, and bottom right: two Glauber gluons exchange diagram. The notation for the scattering centers is the following: $\otimes_{1}=\left[x_{1}, q_{1},\left(b_{1}\right)_{i}\right], \otimes_{2}=\left[x_{2}, q_{2},\left(b_{2}\right)_{j}\right]$. The contact limit of the amplitude $A_{1}^{(2) q}$ is also called double Born amplitude.

It is convenient to define the following quantity, since it directly appears in the expression in Eq. (5.8):

$$
\Omega(p, q)=p^{-}-\frac{\left(\boldsymbol{p}_{\perp}-\boldsymbol{q}_{\perp}\right)^{2}-i \varepsilon}{p^{+}} .
$$

Note that the quantity in the Eq. (5.9) depends only on $\boldsymbol{q}_{\perp}$ and $p$ and always leads to a $q^{-}$pole in the propagator Eq. (5.8) which is in the positive imaginary plane.

\subsection{Quark jet}

We start from the first diagram in figure 2, which represents the lowest order diagram for the matrix element in Eq. (5.1):

$$
A_{1}^{(0) q}=\bar{\chi}_{n, p} i J(p) \mathrm{e}^{i p x_{0}} .
$$

For a $k$ Glauber gluon exchange diagram one has a factor of $\frac{\not h}{2}$ for each quark propagator and a factor of $\frac{\vec{h}}{2}$ for each vertex. These factors can be commuted through to the left and simplified:

$$
\bar{\chi}_{n, p}\left(\frac{\not h}{2} \frac{\not h}{2}\right)^{k}=\bar{\chi}_{n, p}\left(\frac{\not h}{2} \frac{\not h}{2}\right)=\bar{\chi}_{n, p},
$$

which means that we can (and will) ignore this factors in the Feynman rules. Squaring the tree level result in Eq. (5.10) we get:

$$
d \sigma \propto \frac{1}{d_{R} d_{T}} \sum_{\text {spin,color }}\left|A_{1}^{(0) q}\right|^{2}=\operatorname{Tr}\left(\frac{\not h}{2} J(p) \bar{J}(p)\right) \bar{n} \cdot p .
$$

It is instructive to examine here the Lorentz structure of the part of the matrix element squared which is not explicitly written in Eq. (5.12). It can be represented completely generally as follows:

$$
J(p) \bar{J}(p)=\sum_{i=1}^{16} a_{i}(p) \Gamma^{i} .
$$

Here, $\Gamma^{i}$ is a basis set of 16 matrices for the Dirac algebra, for example: $\left\{\mathbf{1}, \gamma^{\mu}, \gamma^{5}, \gamma^{\mu} \gamma^{5}, \sigma^{\mu \nu}=\frac{i}{2}\left[\gamma^{\mu}, \gamma^{\nu}\right]\right\}$. Note that $\sigma^{i j}=\epsilon^{i j k} \Sigma^{k}$. By direct inspection one sees that only the $a_{+}(p) \gamma^{+}$part contributes to the spin averaged cross section. 
For a $k$ Glauber gluon exchange amplitude we have ${ }^{9}$ :

$$
A_{\text {coll }}^{(k) q}=\bar{\chi}_{n, p} \int \prod_{m=1}^{k} d \Phi_{m} B^{(k) q} i J\left(p-\sum_{l=1}^{k} q_{l}\right) \mathrm{e}^{i p x_{0}},
$$

where $B^{(k) q}$ is the non-trivial part of the amplitude given by the $\operatorname{SCET}_{\mathrm{G}}$ Lagrangian. With the notation defined above and using the Feynman rules derived in section 4 and in appendix $\mathrm{C}$ we get in the $R_{\xi}$ gauge:

$$
\begin{aligned}
& B_{1}^{(0) q}=1, \\
& B_{1}^{(1) q}=\left(b_{1}\right)_{R}\left(b_{1}\right)_{T_{i}} i i \Delta_{g}\left(p, q_{1}\right), \\
& B_{1}^{(2) q}=\left(b_{2} b_{1}\right)_{R}\left(b_{1}\right)_{T_{i}}\left(b_{2}\right)_{T_{j}} i i \Delta_{g}\left(p, q_{2}\right) i i \Delta_{g}\left(p, q_{1}+q_{2}\right) .
\end{aligned}
$$

We have absorbed all the factors $\tilde{v}\left(\boldsymbol{q}_{\perp}\right)$ and the phases into the conveniently defined differentials in Eq. (5.3). Next we need to perform the longitudinal integrals $d q^{+}, d q^{-}$in the one and two Glauber gluons exchange diagrams and reduce the integration to only transverse components of $\boldsymbol{q}_{i \perp}$. For the single Born diagram this is done by using the result for the longitudinal integral $I_{1}^{(1)}$ from appendix D.1. The result is:

$$
\int d \Phi_{1} B_{1}^{(1) q}=i(b)_{R}(b)_{T_{i}} \int d \Phi_{1}^{\perp}\left[\mathrm{e}^{i \omega_{1} \delta z_{1}}\right] .
$$

Similarly, using the results from appendix D.1 for the integrals $I_{1}^{(2)}$ and its contact limit $I_{1}^{(2 c)}$, we get the following results for the two Glauber gluons exchange diagram and its contact limit:

$$
\begin{aligned}
& \int d \Phi_{1} d \Phi_{2} B_{2}^{(2) q}=\left(b_{2} b_{1}\right)_{R}\left(b_{1}\right)_{T_{i}}\left(b_{2}\right)_{T_{j}} \int d \boldsymbol{\Phi}_{1}^{\perp} d \boldsymbol{\Phi}_{2}^{\perp}\left[\mathrm{e}^{i\left(\omega_{12} \delta z_{1}+\omega_{2}\left(\delta z_{2}-\delta z_{1}\right)\right)}\right](-1), \\
& \int d \Phi_{1} d \Phi_{2} B_{2}^{(2 c) q}=\left(b_{2} b_{1}\right)_{R}\left(b_{1} b_{2}\right)_{T_{i}} \int d \boldsymbol{\Phi}_{1}^{\perp} d \boldsymbol{\Phi}_{2}^{\perp}\left[\mathrm{e}^{i\left(\omega_{12} \delta z_{1}\right)}\right] \times\left(-\frac{1}{2}\right) .
\end{aligned}
$$

In Eq. (5.18)-Eq. (5.20), the inverse formation times $\omega_{1,2}$ are defined as follows:

$$
\omega_{1}=\Omega\left(p, q_{1}\right), \quad \omega_{2}=\Omega\left(p, q_{2}\right), \quad \omega_{12}=\Omega\left(p, q_{1}+q_{2}\right) .
$$

Finally, combining together the three amplitudes, squaring them and identifying the contribution to first order in opacity, we get:

$$
\frac{1}{d_{R} d_{T}} \operatorname{Tr}\left|A_{1}^{(0) q}+A_{1}^{(1) q}+A_{1}^{(2 c) q}+\ldots\right|^{2}=\frac{1}{d_{R} d_{T}} \operatorname{Tr}\left(\left|A_{1}^{(0) q}\right|^{2}+\left|A_{1}^{(1) q}\right|^{2}+2 \operatorname{Re}\left(A_{1}^{(0) q}\right)^{\dagger} A_{1}^{(2 c) q}+\ldots\right) .
$$

Here, we have omitted the $\operatorname{Re}\left(A_{1}^{(0) q}\right)^{\dagger} A_{1}^{(1) q}$ term, since it vanishes because of the color trace: $\operatorname{Tr}\left(T^{b}(R)\right)=0$. Squaring the $A_{1}^{(0) q}$ term has been performed in Eq. (5.12) and represents the squared matrix element of the production of the quark jet from the underlying hard process $J$. The same exact overall factor, the differential jet distribution $d^{2} \sigma_{q, g} / d^{2} \boldsymbol{p}_{\perp}$, appears in all other terms in Eq. (5.22). We will drop this factor for brevity but will keep in mind that the results derived below should be understood as operators acting on the unperturbed by the medium jet distribution. Thus, the first term becomes simply unity:

$$
\frac{1}{d_{R} d_{T}} \operatorname{Tr}\left|A_{1}^{(0) q}\right|^{2}=1
$$

\footnotetext{
${ }^{9}$ In our notation $B^{(k) q}, B$ stands for "Broadening".
} 
Squaring the single Born amplitude $A^{(1) q}$ we get:

$$
\frac{1}{d_{R} d_{T}} \operatorname{Tr}\left|A_{1}^{(1) q}\right|^{2}=\frac{1}{d_{R} d_{T}} \sum_{i=1}^{N} \operatorname{Tr}\left((b)_{R}\left(b^{\prime}\right)_{R}\right) \operatorname{Tr}\left((b)_{T_{i}}\left(b^{\prime}\right)_{T_{i}}\right) \int d \boldsymbol{\Phi}_{\perp}\left(\boldsymbol{q}_{i}\right) d \boldsymbol{\Phi}_{\perp}\left(\boldsymbol{q}_{i}^{\prime}\right)^{*} \mathrm{e}^{i\left(\omega_{1}\left(q_{i \perp}\right)-\omega_{1}\left(q_{i \perp}^{\prime}\right)\right) \delta z_{i}} .
$$

This expression can be simplified further by turning the sum over the scattering centers into a continuous integral. This approximation is valid if $A_{\perp} \gg \mu^{-2}$ :

$$
\sum_{i=1}^{N} \mathrm{e}^{-i \boldsymbol{p}_{\perp} \delta x_{i}} \approx N \int \frac{d^{2} \boldsymbol{b}}{A_{\perp}} \mathrm{e}^{-i \boldsymbol{p}_{\perp} \cdot \boldsymbol{b}}=N \frac{(2 \pi)^{2} \delta\left(\boldsymbol{p}_{\perp}\right)}{A_{\perp}} .
$$

Using this representation and integrating over $\boldsymbol{q}_{i \perp}^{\prime}$, we obtain a particularly simple expression:

$$
\frac{1}{d_{R} d_{T}} \operatorname{Tr}\left|A_{1}^{(1) q}\right|^{2}=\frac{N}{A_{\perp}} \frac{C_{2}(R) C_{2}(T)}{d_{A}} \int \frac{d^{2} \boldsymbol{q}_{\perp}}{(2 \pi)^{2}}\left|\tilde{v}\left(\boldsymbol{q}_{\perp}\right)\right|^{2} \times e^{-\mathbf{q}_{\perp} \cdot \vec{\nabla}_{\mathbf{p}_{\perp}}}
$$

In Eq. (5.26) $e^{-\mathbf{q}_{\perp} \cdot \vec{\nabla}_{\mathbf{p}_{\perp}}}$ indicates the shift in the transverse momentum of the initial jet distribution. The contribution of the double Born contact term to the first order in opacity cross section correction becomes after a similar averaging procedure, which sets $\boldsymbol{q}_{1 \perp}+\boldsymbol{q}_{2 \perp}=0$ in Eq. (5.20):

$$
\frac{1}{d_{R} d_{T}} \operatorname{Tr}\left(A_{1}^{(0) q}\right)^{\dagger} A_{1}^{(2 c) q}=\left(-\frac{1}{2}\right) \frac{N}{A_{\perp}} \frac{C_{2}(R) C_{2}(T)}{d_{A}} \int \frac{d^{2} \boldsymbol{q}_{\perp}}{(2 \pi)^{2}}\left|\tilde{v}\left(\boldsymbol{q}_{\perp}\right)\right|^{2} .
$$

Note that in Eq. (5.27) there is no net transverse momentum transfer to the jet and no momentum shift. Combining the contributions up to first order in opacity we finally get:

$$
\begin{aligned}
\frac{1}{d_{R} d_{T}} \operatorname{Tr}\left(\left|A_{1}^{(0) q}\right|^{2}\right. & \left.+\left|A_{1}^{(1) q}\right|^{2}+2 \operatorname{Re}\left(A_{1}^{(0) q}\right)^{\dagger} A_{1}^{(2 c) q}\right) \\
& =1+\frac{N}{A_{\perp}} \int d^{2} \boldsymbol{q}_{\perp}\left[\frac{d \sigma_{\mathrm{el}}(R, T)}{d^{2} \boldsymbol{q}_{\perp}} e^{\left.-\mathbf{q}_{\perp} \cdot \vec{\nabla}_{\mathbf{p}_{\perp}}-\sigma_{\mathrm{el}} \delta^{(2)}\left(\boldsymbol{q}_{\perp}\right)\right]}\right.
\end{aligned}
$$

where we expressed the answer through the elastic scattering, which in the lowest Born approximation equals to:

$$
\frac{d \sigma_{\mathrm{el}}(R, T)}{d^{2} \boldsymbol{q}_{\perp}}=\frac{C_{2}(R) C_{2}(T)}{d_{A}} \frac{\left|\tilde{v}\left(\boldsymbol{q}_{\perp}\right)\right|^{2}}{(2 \pi)^{2}}
$$

\subsection{Gluon jet}

We repeat a similar calculation as in the previous subsection for the gluon jet scattering off a fermionic scattering center. The first diagram in figure 3 equals to:

$$
A_{1}^{(0) g, b}=\mathrm{e}^{i p x_{0}} i J_{\nu, a}(p) \varepsilon^{\nu}(p) \delta^{a b} .
$$

The square of this matrix element describes the hard scattering production cross section and appears in every term and is dropped for brevity below. For $k$ Glauber gluon exchange with medium amplitude we use the following notation, similarly to the quark case above in Eq. (5.14):

$$
A_{\text {coll }, b}^{(k) g}=\varepsilon^{\nu}(p) \int \prod_{m=1}^{k} d \Phi_{m} B_{\mu \nu, a b}^{(k) g} i J^{\mu, a}\left(p-\sum_{l=1}^{k} q_{l}\right) \mathrm{e}^{i p x_{0}} .
$$




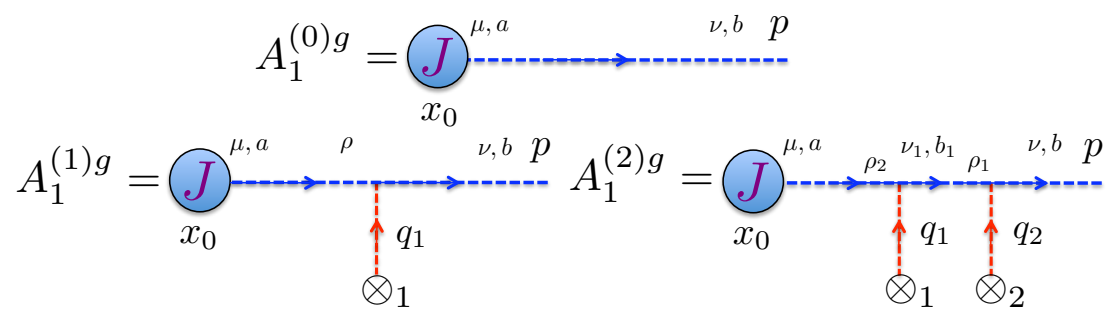

Figure 3: Three lowest order diagrams contributing to the gluon jet in-medium interaction. Top: tree level, bottom left: single Born diagram, and bottom right: two single Born exchanges diagram. The notation for the scattering centers is the following: $\otimes_{1}=\left[x_{1}, q_{1},\left(c_{1}\right)_{i}\right], \otimes_{2}=\left[x_{2}, q_{2},\left(c_{2}\right)_{j}\right]$.

With this notation, using the Feynman rules of $\mathrm{SCET}_{\mathrm{G}}$ in covariant gauge we get for $B^{(k) g}$ amplitudes for $k=0,1,2$ :

$$
\begin{aligned}
\left(B_{1}^{(0) g}\right)^{\mu \nu, a b}= & g^{\mu \nu} \delta^{a b} \\
\left(B_{1}^{(1) g}\right)^{\mu \nu, a b}= & f^{a b c_{1}}\left(c_{1}\right)_{T_{i}} \tilde{\Sigma}_{1}^{\rho \nu}\left(p-q_{1}, p\right)(-i) \Delta(p-q)_{\left(R_{\xi}\right)}^{\mu \rho} \\
\left(B_{1}^{(2) g}\right)^{\mu \nu, a b}= & f^{b_{1} b c_{2}}\left(c_{2}\right)_{T_{j}} \tilde{\Sigma}_{1}^{\rho_{1} \nu}\left(p-q_{2}, p\right)(-i) \Delta\left(p-q_{2}\right)_{\left(R_{\xi}\right)}^{\nu_{1} \rho_{1}} f^{a b_{1} c_{1}}\left(c_{1}\right)_{T_{i}} \\
& \times \tilde{\Sigma}_{1}^{\rho_{2} \nu_{1}}\left(p-q_{1}-q_{2}, p-q_{2}\right)(-i) \Delta\left(p-q_{1}-q_{2}\right)_{\left(R_{\xi}\right)}^{\mu \rho_{2}} .
\end{aligned}
$$

In the Equations Eq. (5.33)-Eq. (5.34), $\Delta(p)_{\left(R_{\xi}\right)}^{\mu \nu}$ is a standard gluon propagator in the covariant gauge, and the $\tilde{\Sigma}_{1}^{\mu \nu}\left(p, p^{\prime}\right)$ is the effective Feynman rule for covariant gauge for Glauber gluon coupling to collinear gluons. As one can see from our appendix C (also from section 4) it reads:

$$
\tilde{\Sigma}_{1}^{\mu \nu}\left(p, p^{\prime}\right) \equiv g^{\mu \nu} \bar{n} \cdot p+\bar{n}^{\mu}\left(p^{\prime}-p\right)_{\perp}^{\nu}-\bar{n}^{\nu}\left(p^{\prime}-p\right)_{\perp}^{\mu}-\frac{1-\frac{1}{\xi}}{2}\left(\bar{n}^{\nu} p^{\mu}+\bar{n}^{\mu} p^{\prime \nu}\right) .
$$

To work out the contraction of vertices for single and double Born diagrams, the following identities are useful:

$$
\begin{gathered}
\varepsilon_{\nu}(p) \tilde{\Sigma}_{1}^{\rho \nu}\left(p-q_{1}, p\right) N_{\mu \rho}^{\left(R_{\xi}\right)}\left(p-q_{1}\right)=\boldsymbol{\varepsilon}_{\perp} \cdot\left(\boldsymbol{p}_{\perp}-\boldsymbol{q}_{1 \perp}\right) \bar{n}_{\mu}+\varepsilon_{\mu}^{\perp} \bar{n} \cdot p \\
\varepsilon_{\nu}(p) \tilde{\Sigma}_{1}^{\rho_{1} \nu}\left(p-q_{2}, p\right) N_{\nu_{1} \rho}^{\left(R_{\xi}\right)}\left(p-q_{2}\right) \tilde{\Sigma}_{1}^{\rho_{2} \nu_{1}}\left(p-q_{1}-q_{2}, p-q_{2}\right) N_{\mu \rho 2}^{\left(R_{\xi}\right)}\left(p-q_{1}-q_{2}\right) \\
=\bar{n} \cdot p \boldsymbol{\varepsilon}_{\perp} \cdot\left(\boldsymbol{p}_{\perp}-\boldsymbol{q}_{1 \perp}-\boldsymbol{q}_{2 \perp}\right) \bar{n}_{\mu}+(\bar{n} \cdot p)^{2} \varepsilon_{\mu}^{\perp},
\end{gathered}
$$

where we defined $N^{\left(R_{\xi}\right)}$ to be the numerator of the covariant gauge gluon propagator:

$$
N_{\mu \nu}^{\left(R_{\xi}\right)}(p)=g_{\mu \nu}-\frac{p_{\mu} p_{\nu}}{p^{2}}(1-\xi) .
$$

Note that even though we work in the covariant gauge, we are free to choose any polarization vector for the on-shell final state gluon. We assume for the external scattered gluon as well as its source the physical polarizations:

$$
\begin{aligned}
\bar{n} \cdot \varepsilon(p) & =0, & & p \cdot \varepsilon(p)=0, \\
\bar{n} \cdot J(p-q) & =0, & & (p-q) \cdot J(p-q)=0 .
\end{aligned}
$$

Equation (5.39) was the only assumption in deriving Eqs. (5.36, 5.37) above. If in addition one assumes Eqs. (5.40), then the first terms can be dropped in each of the Eqs. $(5.36,5.37)$ and only the transverse 
term survives. Under these conditions and using results for the same longitudinal integral as in the quark case, which are summarized in the appendix D.1, we get for the single Born amplitude ${ }^{10}$ :

$$
\int d \Phi_{1}\left(B_{1}^{(1) g}\right)^{\mu \nu, a b}=-f^{a b c_{1}}\left(c_{1}\right)_{T_{i}}\left(g_{\perp}^{\mu \nu}\right) \int d \mathbf{\Phi}_{1 \perp}\left[\mathrm{e}^{i \omega_{1} \delta z_{1}}\right]
$$

which differs from the quark case in Eq. (5.18) only by a color and Lorentz structure. Similarly for the two Glauber gluons exchange diagram and its contact limit we obtain the following results:

$$
\begin{aligned}
& \int d \Phi_{1} d \Phi_{2}\left(B_{1}^{(2) g}\right)^{\mu \nu, a b}=f^{a b_{1} c_{1}} f^{b b_{1} c_{2}}\left(c_{2}\right)_{T_{j}}\left(c_{1}\right)_{T_{i}}\left(g_{\perp}^{\mu \nu}\right) \int d \boldsymbol{\Phi}_{1}^{\perp} d \boldsymbol{\Phi}_{2}^{\perp}\left[\mathrm{e}^{i\left(\omega_{12} \delta z_{1}+\omega_{2}\left(\delta z_{2}-\delta z_{1}\right)\right)}\right](-1) \\
& \int d \Phi_{1} d \Phi_{2}\left(B_{1}^{(2 c) g}\right)^{\mu \nu, a b}=f^{a b_{1} c_{1}} f^{b b_{1} c_{2}}\left(c_{2}\right)_{T_{i}}\left(c_{1}\right)_{T_{i}}\left(g_{\perp}^{\mu \nu}\right) \int d \boldsymbol{\Phi}_{1}^{\perp} d \boldsymbol{\Phi}_{2}^{\perp}\left[\mathrm{e}^{i\left(\omega_{12} \delta z_{1}\right)}\right] \times\left(-\frac{1}{2}\right) .
\end{aligned}
$$

The final result for the cross section to lowest order in opacity coincides with the one obtained in the last subsection for the quark case, with the substitution of the gluon-quark cross section instead of quark-quark elastic scattering cross section. Combining the contributions up to first order in opacity we finally get:

$$
\begin{aligned}
\frac{1}{d_{R} d_{T}} \operatorname{Tr}\left(\left|A_{1}^{(0) g}\right|^{2}\right. & \left.+\left|A_{1}^{(1) g}\right|^{2}+2 \operatorname{Re}\left(A_{1}^{(0) g}\right)^{\dagger} A_{1}^{(2 c) g}\right) \\
& =1+\frac{N}{A_{\perp}} \int d^{2} \boldsymbol{q}_{\perp}\left[\frac{d \sigma_{\mathrm{el}}(R, T)}{d^{2} \boldsymbol{q}_{\perp}} e^{\left.-\mathbf{q}_{\perp} \cdot \vec{\nabla}_{\mathbf{p}_{\perp}}-\sigma_{\mathrm{el}} \delta^{(2)}\left(\boldsymbol{q}_{\perp}\right)\right] .}\right.
\end{aligned}
$$

Here,

$$
\frac{d \sigma_{\mathrm{el}}^{(g)}(R, T)}{d^{2} \boldsymbol{q}_{\perp}}=\frac{C_{2}(R) C_{2}(T)}{d_{A}} \frac{\left|\tilde{v}\left(\boldsymbol{q}_{\perp}\right)\right|^{2}}{(2 \pi)^{2}} .
$$

In this case the $R$ stands for adjoint representation (gluon jet) and $T$ stands for fundamental representation (fermionic static center).

\section{Medium-induced bremsstrahlung}

In this section we use the Feynman rules of $\mathrm{SCET}_{\mathrm{G}}$ to derive the probability for an energetic quark to emit a gluon, induced by the jet interactions in QCD matter. This is equivalent to evaluating the differential distribution of the number of emitted gluons. We first present this calculation in the vacuum using SCET and later in the medium using the new $\mathrm{SCET}_{\mathrm{G}}$ Lagrangian. In each case we consider the covariant gauge and the initially static source. We also focus on final-state (FS) radiation. In the literature, such a calculation is typically done in the soft (emitted) gluon approximation. However, in SCET and $\mathrm{SCET}_{\mathrm{G}}$ dynamics, the leading interaction describes the collinear gluon emission, which will allow us to go easily beyond the conventional $\omega \ll E$ limit. We will perform this new calculation below in section 9 , while in this section we will focus on taking the soft gluon limit and comparing to the previously derived results for radiative energy loss in QCD matter.

$$
A_{\text {brem }}=\left\langle J\left|T \bar{\chi}_{n}\left(x_{0}\right) \mathrm{e}^{i \int d^{4} x\left(\mathcal{L}^{\mathrm{QCD}}+\mathcal{L}^{\mathrm{SCET}_{\mathrm{G}}}\right)}\right| \mathbf{p}, \mathbf{k}\right\rangle .
$$

\footnotetext{
${ }^{10}$ Note that in our notation the covariant gauge gluon propagator of collinear plus Glauber momentum can be written as $\Delta(p-q)_{\left(R_{\xi}\right)}^{\mu \nu}=N_{\left(R_{\xi}\right)}^{\mu \nu}(p-q) \Delta_{g}(p, q) \frac{1}{\bar{n} p}$. Using this identity along with Eqs. $(5.36,5.37)$ leads to the same results for the broadening of gluon jet as for the quark jet up to the color and Dirac structure.
} 

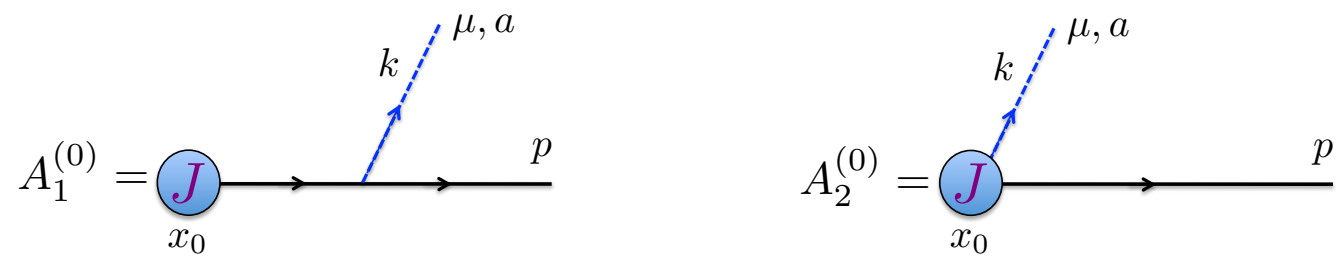

Figure 4: Diagrams in SCET describing splitting of a collinear quark into a collinear quark and a collinear gluon.

To study gluon emission, we start from the matrix element, Eq. (6.1), where $J$ is the underlying hard process that creates the quark jet, $\bar{\chi}_{n}$ is the gauge invariant quark field, and $p, k$ are the momenta of the final state quark and of the emitted gluon, correspondingly. Since in this section we consider only the case of the initial quark jet, we omit the quark index in the amplitudes below for brevity. The matrix element in Eq. (6.1) gets contributions from 0,1,2,.. Glauber gluon exchanges between the collinear quark and/or gluon and the sources in the medium. The first three correspond to vacuum emission, single Born amplitude and two single Born exchanges amplitude, respectively, and are calculated in the subsections below. To simplify the notation we write the $n$-Glauber insertion amplitude in the following form ${ }^{11}$ :

$$
A^{(n), a}=g \bar{\chi}_{n, p}\left(\prod_{l=1}^{n} \int d \Phi_{l}\right) R\left(q_{1}, \ldots, q_{n}\right)^{(n) \mu, a} i J\left(k+p-\sum_{k=1}^{n} q_{k}\right) \mathrm{e}^{i(k+p) x_{0}} \varepsilon_{\mu}(k) .
$$

\subsection{Obtaining the Altarelli-Parisi splitting function in SCET}

A large $Q^{2}$ process is accompanied by bremsstrahlung even in the absence of in-medium interactions. Knowledge of the corresponding amplitudes is also essential for the evaluation of the interference effects between the different sources or radiation for jet production in the QCD medium.

Calculation of the vacuum diagrams in figure 4 leads to the Altarelli-Parisi splitting function for the $q \rightarrow q g$ process. This calculation has been performed in Ref. [52] in the light-cone gauge. We perform the same calculation here in the covariant gauge. We also demonstrate how in the small $x=k^{+} / p^{+}$limit the relevant radiation piece can be identified at the amplitude level.

The calculation of the relevant radiative matrix element can be performed using different set of fields in SCET (see [53]): gauge dependent fields $\xi_{n}, A_{n}$ or gauge independent ones $\chi_{n}$ and $\mathcal{B}_{n}$. In the former case the Feynman rules contain Wilson line emissions, from the gauge invariant quark field $\chi_{n}=W_{n}^{\dagger} \xi_{n}$. In the latter case these diagrams are absent, since we do calculations directly with $\chi_{n}$. However, the difference in the second case is that the SCET Lagrangian is modified and contains explicit collinear Wilson lines $W_{n}$ which changes the Feynman rules. The equivalence of different formulations was shown in [53] on the example of the quark jet function at one loop. We choose the first case, i.e. use fields $\xi_{n}, A_{n}$ to do the calculation and include the Wilson line graphs, while keeping the SCET Lagrangian free from any collinear Wilson lines. In order to avoid confusion, we note that for the external quark spinor we use $\bar{\chi}_{n}$ as before for the broadening, which is simply a matter of notation.

We first recall that for the jet production amplitude we have:

$$
A_{J q}=\bar{\chi}_{n, p} i J \mathrm{e}^{i p x_{0}}, \quad \frac{1}{d_{R} d_{T}} \sum_{\text {spin, color }} d \sigma \propto\left|A_{J q}\right|^{2}=\operatorname{Tr}\left(\frac{\not h}{2} \bar{n} \cdot p J(p) \bar{J}(p)\right) .
$$

\footnotetext{
${ }^{11}$ In our notation $R^{\mu}$ stands for "Radiation".
} 
One notices from Eq. (6.3) that the contribution to the cross section comes from the part $J(p) \bar{J}(p) \propto \gamma^{+}$. For the case of gluon bremsstrahlung, the first amplitude in figure 4 reads:

$$
R_{1}^{(0) \mu, a}=i T^{a}\left(n^{\mu}+\frac{\gamma_{\perp}^{\mu}\left(\not p_{\perp}+\not \ell_{\perp}\right)}{\bar{n} \cdot(p+k)}+\frac{\not p_{\perp} \gamma_{\perp}^{\mu}}{\bar{n} \cdot p}-\frac{\not p_{\perp}(\not p+\not k)_{\perp}}{\bar{n} \cdot p \bar{n} \cdot(p+k)} \bar{n}^{\mu}\right) i \frac{\bar{n}(p+k)}{(p+k)^{2}} .
$$

Even though we work in the covariant gauge, we are going to use physical polarization vectors for the emitted real gluon. We choose the following gluon polarization vectors, which are the only possible solutions that satisfy the two conditions $\bar{n} \cdot \varepsilon=0, k \cdot \varepsilon(k)=0$ :

$$
\varepsilon_{i}^{\mu}(k)=\left(0, \frac{2 \varepsilon_{i \perp} \cdot \mathbf{k}_{\perp}}{k^{+}}, \varepsilon_{i \perp}\right), \quad i=1,2 .
$$

For such polarization vectors the second diagram in figure 4 vanishes, since it is proportional to $\bar{n}^{\mu}$, and also the last term in Eq. (6.4) vanishes once dotted with the polarization vector. By contracting the polarization vectors from Eq. (6.5) with the SCET amplitude in Eq. (6.4), we get ${ }^{12}$ :

$$
\begin{aligned}
& R_{1}^{(0) \mu, a} \varepsilon_{\mu}=-T^{a}\left[\frac{2 \boldsymbol{A}_{\perp}^{i}}{\boldsymbol{A}_{\perp}^{2}}+\frac{x}{\boldsymbol{A}_{\perp}^{2}} \boldsymbol{A}_{\perp}^{j} \gamma_{\perp}^{i} \gamma_{\perp}^{j}\right] \varepsilon_{\perp}^{i}, \\
& \boldsymbol{A}_{\perp} \equiv \boldsymbol{k}_{\perp}(1-x)-\boldsymbol{p}_{\perp} x
\end{aligned}
$$

where $x$ is the fraction of energy taken by the emitted gluon $k^{+}=x p_{0}^{+}, p^{+}=(1-x) p_{0}^{+}$. To derive Eq. (6.6) we also assumed that the external quark and gluon are on-shell. Squaring this amplitude, averaging over the colors of the radiating quark we obtain:

$$
\begin{aligned}
& \frac{1}{d_{R}}\left|A_{J q \rightarrow q g}\right|^{2}=\frac{g^{2}}{d_{R}} \operatorname{Tr}\left(\frac{\not h}{2} \bar{n} \cdot p J \bar{J}\left[\gamma^{0}\left(R_{1}^{(0)} \cdot \varepsilon\right)^{\dagger} \gamma^{0}\left(R_{1}^{(0)} \cdot \varepsilon\right)\right]\right), \\
& \gamma^{0}\left(R_{1}^{(0)} \cdot \varepsilon\right)^{\dagger} \gamma^{0}\left(R_{1}^{(0)} \cdot \varepsilon\right)=4 C_{F}\left(1-x+\frac{x^{2}}{2}\right) \frac{1}{\boldsymbol{A}_{\perp}^{2}}(\mathbb{I})_{\text {color }}(\mathbb{I})_{\text {Dirac }} .
\end{aligned}
$$

The fact that the expression in Eq. (6.9) is a Dirac scalar means that the cross section of splitting factorizes into the cross section of jet production times the Altarelli-Parisi kernel, which has a nice probabilistic interpretation. Setting the light-cone direction along the initial quark before splitting, i.e. $\boldsymbol{p}_{\perp}=-\boldsymbol{k}_{\perp}$, we reproduce the Altarelli-Parisi kernel for $q \rightarrow q g$ splitting:

$$
\frac{1}{d_{R}}\left|A_{J q \rightarrow q g}\right|^{2}=(1-x) \operatorname{Tr}\left(\frac{\not h}{2} p_{0}^{+} J(0) \bar{J}(0)\right) \times 4 g^{2} C_{F}\left(1-x+\frac{x^{2}}{2}\right) \frac{1}{\boldsymbol{k}_{\perp}^{2}}=\left|A_{J q}\right|^{2} \times\left|M_{0}^{\mathrm{rad}}\right|^{2} .
$$

We note that the $1-x$ factor is associated with the reduction of the cross section to have a high momentum final-state quark when a gluon is emitted. From Eq. (6.10) we can identify the radiative correction and supplying the one body phase space, written as $d k^{+} d^{2} \boldsymbol{k}_{\perp} /(2 \pi)^{3} 2 k^{+}$, we obtain:

$$
\frac{d N^{g}}{d x d^{2} \boldsymbol{k}_{\perp}}=C_{F} \frac{\alpha_{s}}{\pi^{2}} \frac{\left(1-x+\frac{x^{2}}{2}\right)}{x} \frac{1}{\boldsymbol{k}_{\perp}^{2}} .
$$

One easily recognizes in Eq. (6.11) the usual Altarelli-Parisi splitting probability for quarks (up to $x \rightarrow$ $1-x)$. Taking the small- $x$ limit one can conveniently reproduce the boundary condition [40] associated

\footnotetext{
${ }^{12}$ Note that in Eq. (6.6) all indices are contravariant, while for example terms in Eq. (6.4) contain contractions between covariant and contravariant vectors, like $p_{\perp} \equiv p^{\mu} \gamma_{\perp \mu}=-\boldsymbol{p}_{\perp}^{i} \gamma_{\perp}^{i}$.
} 


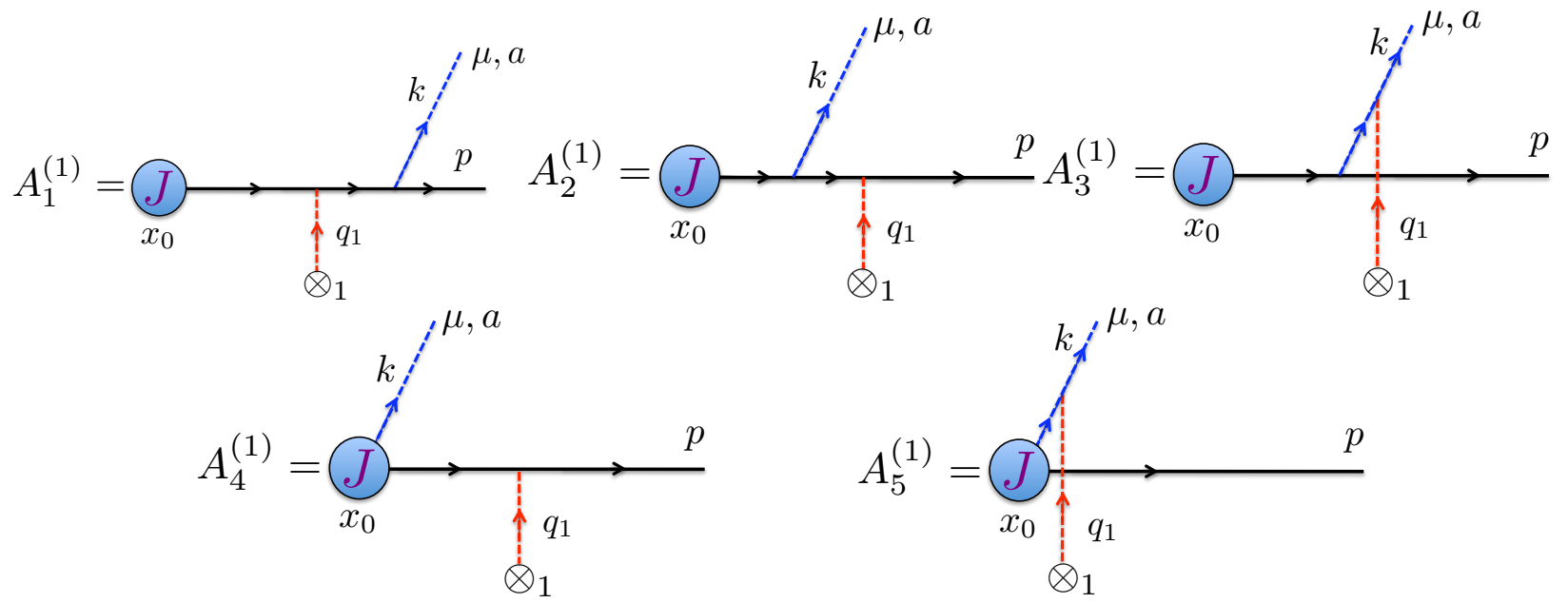

Figure 5: Single Born diagrams contributing to the medium-induced gluon bremsstrahlung Eq. (6.2). The notation for the scattering centers is the following: $\otimes_{1}=\left[x_{1}, q_{1},\left(b_{1}\right)_{i}\right]$.

with hard jet production that is subsequently used in the reaction operator approach to parton energy loss $[40,51]$.

We finally point out that in the soft gluon limit $x \ll 1$ the amplitude itself, Eq. (6.6), reduces as follows:

$$
A_{J q \rightarrow q g} \approx \mathrm{e}^{i p x_{0}} \bar{\chi}_{n, p} J\left(-g \frac{2 \varepsilon_{i \perp} \cdot \boldsymbol{k}_{\perp}}{\boldsymbol{k}_{\perp}^{2}}\right) T^{a}=A_{J q} \times M_{0}^{\mathrm{rad}}
$$

and, just like in quantum electrodynamics in the soft photon limit, a radiation matrix element can be identified at the amplitude level:

$$
M_{0}^{\mathrm{rad}}=-g \frac{2 \varepsilon_{i \perp} \cdot \boldsymbol{k}_{\perp}}{\boldsymbol{k}_{\perp}^{2}} T^{a}
$$

\subsection{Single Born amplitudes in $\mathrm{SCET}_{\mathrm{G}}$}

In this subsection we derive the single Born amplitudes in the fully covariant gauge for the scattering off the initially static source. In figure 5 we list all the relevant single Born diagrams. In this gauge two additional diagrams $A_{4}, A_{5}$ appear when the collinear gluon appears from the Wilson line $W^{\dagger}$. Feynman rule for such collinear gluon vertex is well known:

$$
\Gamma_{W}^{\alpha, a}(k)=g T_{r}^{a} \frac{\bar{n}^{\alpha}}{k^{+}+i \epsilon}
$$

where $k$ is the outgoing gluon momentum from the Wilson line. Although we use the $R_{\xi}$ gauge in this subsection, just as in the vacuum case above, we use the physical gluon polarization vector for the emitted gluon Eq. (6.5). With the Feynman rules of $\mathrm{SCET}_{\mathrm{G}}$, derived in section 4, and the notation of Eq. (6.2), 
we get the following expressions for the amplitudes in figure 5:

$$
\begin{aligned}
R_{1}^{(1) \mu, a}= & i(a)_{R}\left(n^{\mu}+\frac{\gamma_{\perp}^{\mu}\left(\not p_{\perp}+\not k_{\perp}\right)}{p^{+}+k^{+}}+\frac{\not p_{\perp} \gamma_{\perp}^{\mu}}{p^{+}}\right) \frac{i\left(p^{+}+k^{+}\right)}{(p+k)^{2}+i \varepsilon} i\left(b_{1}\right)_{R}\left(b_{1}\right)_{T_{i}} i \Delta_{g}\left(p+k, q_{1}\right), \\
R_{2}^{(1) \mu, a}= & i\left(b_{1}\right)_{R}\left(b_{1}\right)_{T_{i}} i \Delta_{g}\left(p, q_{1}\right) i(a)_{R}\left(n^{\mu}+\frac{\gamma_{\perp}^{\mu}\left(p_{\perp}+\not k_{\perp}-\not q_{1 \perp}\right)}{p^{+}}+\frac{\left(\not p_{\perp}-\not q_{1 \perp}\right) \gamma_{\perp}^{\mu}}{p^{+}}\right) i \Delta_{g}\left(p+k, q_{1}\right), \\
R_{3}^{(1) \mu, a}= & i\left(c_{1}\right)_{R}\left(n^{\rho_{1}}+\frac{\gamma_{\perp}^{\rho_{1}}\left(\not p_{\perp}+\not k_{\perp}-\not \not_{1}\right)}{p^{+}}+\frac{\not p_{\perp} \gamma_{\perp}^{\rho_{1}}}{p^{+}}\right) \\
& \times \frac{(-i) \Delta_{g}\left(k, q_{1}\right)}{\bar{n} \cdot k} N_{\rho_{1} \rho 2}^{\left(R_{\xi}\right)}\left(k-q_{1}\right) i \Delta_{g}\left(p+k, q_{1}\right) f^{c_{1} a b_{1}}\left(b_{1}\right)_{T_{i}} \tilde{\Sigma}_{1}^{\rho_{2} \mu}\left(k-q_{1}, k\right), \\
R_{4}^{(1) \mu, a}= & 0 \\
R_{5}^{(1) \mu, a}= & 0 .
\end{aligned}
$$

Note that in the collinear gluon vertices in diagrams $A_{1}, A_{2}$ we omitted the last term proportional to $\bar{n}^{\mu}$ because after contraction with the polarization vector of our choice this term vanishes, since $\varepsilon^{+}=0$. For exactly the same reason diagram $A_{4}$ vanishes. However, the reason why we ignored $\bar{n}^{\rho_{1}}$ in diagram $A_{3}$ and why $A_{5}$ vanishes, is slightly more involved. The point is that both $A_{3}$ and $A_{5}$ have a common factor given by Eq. (5.36) with $p \rightarrow k$. Since from this identity it is obvious that $\bar{n}^{\rho_{1}}$ times this combination vanishes, we are allowed to omit this term in $A_{3}$. For the same reason $A_{5}=0$.

In order to reduce the integral $d \Phi_{1} R_{i}^{(1)}$ to the $d \boldsymbol{\Phi}_{1 \perp}$ integral we use the identity in Eq. (5.5). Also, substituting Eq. (5.36) into the expression for $R_{3}^{(1)}$ makes it obvious that the entire dependence on $q_{1}^{-}$ appears through the propagators $\Delta_{g}(p, q)$. Using the form of this propagator from Eq. (5.8) we define the relevant longitudinal integrals $I_{1}^{(1)}, I_{2}^{(1)}, I_{3}^{(1)}$. We evaluate these integrals in appendix D.2. Thus, using Eq. (5.5) and the expressions for $I_{1}^{(1)}, I_{2}^{(1)}, I_{3}^{(1)}$ from appendix D.2, we get after taking limit $x \ll 1$ in $R_{1,2,3}^{(1)}$ :

$$
\begin{aligned}
\int d \Phi_{1} R_{1}^{(1) \mu, a} \varepsilon_{\mu}(k) & \approx(-i)\left(a b_{1}\right)_{R}\left(b_{1}\right)_{T_{i}} \frac{2 \boldsymbol{k}_{\perp} \cdot \boldsymbol{\varepsilon}_{\perp}}{\boldsymbol{k}_{\perp}^{2}} \int d \boldsymbol{\Phi}_{1 \perp} \mathrm{e}^{i \omega_{0} \delta z_{1}}, \\
\int d \Phi_{1} R_{2}^{(1) \mu, a} \varepsilon_{\mu}(k) & \approx(-i)\left(b_{1} a\right)_{R}\left(b_{1}\right)_{T_{i}} \frac{2 \boldsymbol{k}_{\perp} \cdot \boldsymbol{\varepsilon}_{\perp}}{\boldsymbol{k}_{\perp}^{2}} \int d \boldsymbol{\Phi}_{1 \perp}\left[1-\mathrm{e}^{i \omega_{0} \delta z_{1}}\right], \\
\int d \Phi_{1} R_{3}^{(1) \mu, a} \varepsilon_{\mu}(k) & \approx(-i)\left[a, b_{1}\right]_{R}\left(b_{1}\right)_{T_{i}} \int d \boldsymbol{\Phi}_{1 \perp} \frac{2\left(\boldsymbol{k}_{\perp}-\boldsymbol{q}_{1 \perp}\right) \cdot \boldsymbol{\varepsilon}_{\perp}}{\left(\boldsymbol{k}_{\perp}-\boldsymbol{q}_{1 \perp}\right)^{2}} \mathrm{e}^{i \omega_{0} \delta z_{1}}\left[\mathrm{e}^{-i \omega_{1} \delta z_{1}}-1\right] .
\end{aligned}
$$

The two inverse formation times $\omega_{0}$ and $\omega_{1}$ are defined according to:

$$
\omega_{0}=\frac{\boldsymbol{k}_{\perp}^{2}}{x p_{0}^{+}}, \quad \omega_{1}=\frac{\left(\boldsymbol{k}_{\perp}-\boldsymbol{q}_{1 \perp}\right)^{2}}{x p_{0}^{+}} .
$$

\subsection{Double Born amplitudes in $\mathrm{SCET}_{\mathrm{G}}$}

In this section we calculate all diagrams in figure 6 in the $R_{\xi}$ gauge for the initially static source. Expressions for all of these diagrams are obtained directly from the Feynman rules of $\mathrm{SCET}_{\mathrm{G}}$ in the covariant gauge, and are straightforward, though lengthy. However, a nice compact relations can be found for these diagrams by relating them to previously calculated amplitudes $R^{(0)}, R^{(1)}$ and $B^{(1) q}, B^{(2) q}, B^{(1) g}$, $B^{(2) g}$, in the notation 


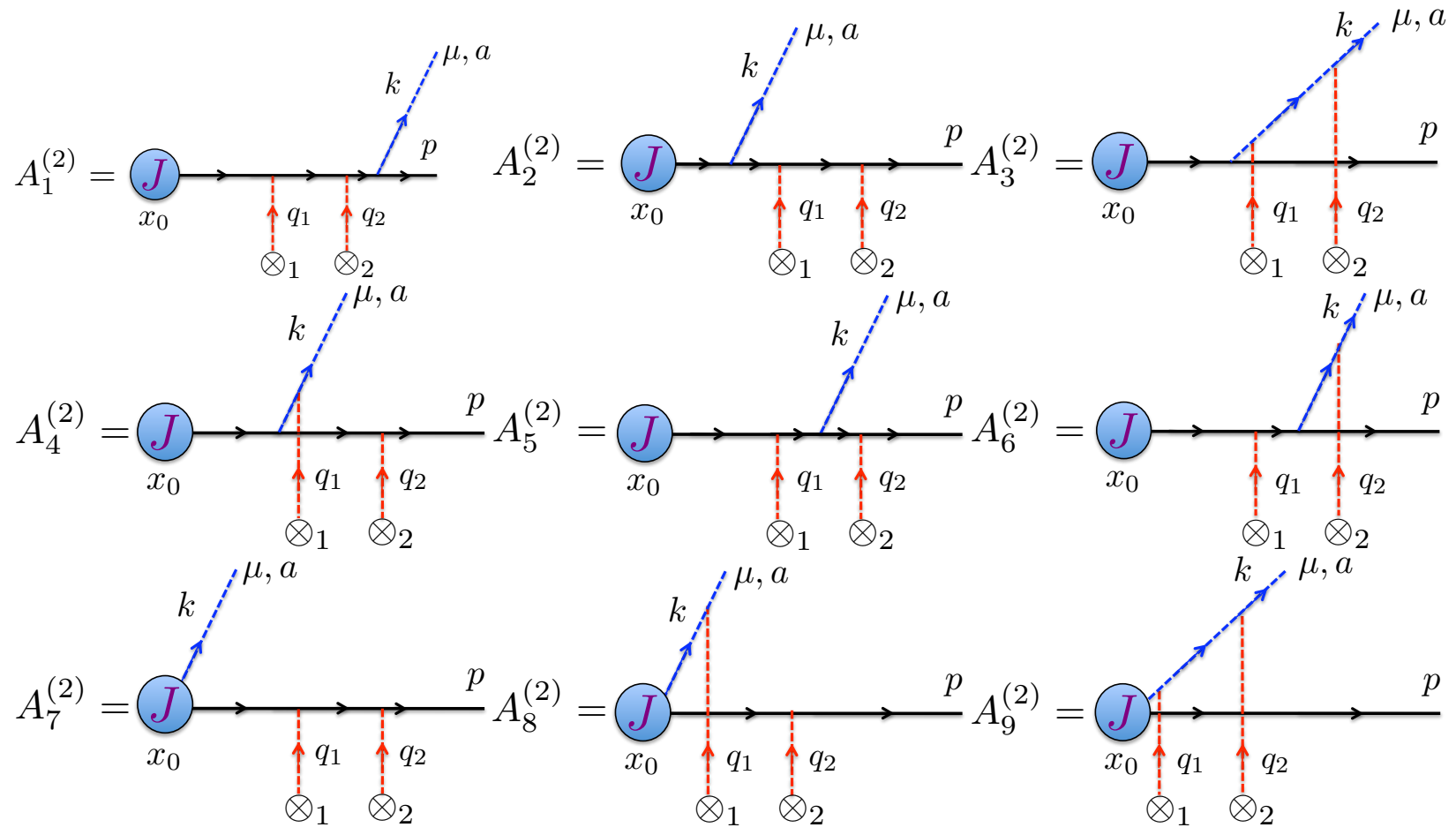

Figure 6: Two single Born exchange diagrams (double Born diagrams in the contact limit) contributing to matrix element in Eq. (6.2). The notation for the scattering centers is the following: $\otimes_{1}=\left[x_{1}, q_{1},\left(b_{1}\right)_{i}\right], \otimes_{2}=\left[x_{2}, q_{2},\left(b_{2}\right)_{j}\right]$.

of Eq. (5.14), Eq. (5.31) and Eq. (6.2). The result is as follows:

$$
\begin{aligned}
& \left(R_{1}^{(2)}\right)^{\mu, a}=R_{1}^{(1) \mu, a}\left(p, k, q_{2}\right) B_{1}^{(1) q}\left(p+k-q_{2}, q_{1}\right), \\
& \left(R_{2}^{(2)}\right)^{\mu, a}=B_{1}^{(1) q}\left(p, q_{2}\right) R_{2}^{(1) \mu, a}\left(p-q_{2}, k, q_{1}\right), \\
& \left(R_{3}^{(2)}\right)^{\mu, a}=R_{1}^{(0) \rho_{1}, b}\left(p, k-q_{1}-q_{2}\right)\left(B_{1}^{(2) g}\right)^{\mu \rho_{1}, b a}\left(k, q_{1}, q_{2}\right), \\
& \left(R_{4}^{(2)}\right)^{\mu, a}=B_{1}^{(1) q}\left(p, q_{2}\right) R_{3}^{(1) \mu, a}\left(p-q_{2}, k, q_{1}\right), \\
& \left(R_{5}^{(2)}\right)^{\mu, a}=R_{2}^{(1) \mu, a}\left(p, k, q_{2}\right) B_{1}^{(1) q}\left(p+k-q_{2}, q_{1}\right), \\
& \left(R_{6}^{(2)}\right)^{\mu, a}=R_{3}^{(1) \mu, a}\left(p, k, q_{2}\right) B_{1}^{(1) q}\left(p+k-q_{2}, q_{1}\right), \\
& \left(R_{7,8,9}^{(2)}\right)^{\mu, a}=0 .
\end{aligned}
$$

Next, we integrate over the longitudinal momenta and reduce the integrals to transverse ones. This is analogous to the procedure for the single Born diagrams. The only poles in $q_{i}^{-}$come from propagators of collinear momenta plus Glauber momenta (given by Eq. (5.8)). We summarize the corresponding integrals in appendix D.2. Since the amplitudes $A_{7,8,9}$ vanish for physical polarization of the radiated gluon, we do not even consider the corresponding longitudinal integrals. Finally, using our results for the corresponding longitudinal integrals $I_{1}^{(2)}, \cdots I_{6}^{(2)}$ from appendix D.2 and taking the $x \ll 1$ approximation, we get the 
following expressions:

$$
\begin{aligned}
& \int d \Phi_{1} d \Phi_{2} R_{1}^{(2) \mu, a} \varepsilon_{\mu}(k)=\left(a b_{2} b_{1}\right)_{R}\left(b_{1}\right)_{T_{i}}\left(b_{2}\right)_{T_{j}} \int d \Phi_{1 \perp} d \Phi_{2 \perp} \frac{2 \boldsymbol{k}_{\perp} \cdot \boldsymbol{\varepsilon}_{\perp}}{\boldsymbol{k}_{\perp}^{2}} \mathrm{e}^{i \omega_{0} \delta z_{2}}, \\
& \int d \Phi_{1} d \Phi_{2} R_{2}^{(2) \mu, a} \varepsilon_{\mu}(k)=\left(b_{2} b_{1} a\right)_{R}\left(b_{1}\right)_{T_{i}}\left(b_{2}\right)_{T_{j}} \int d \boldsymbol{\Phi}_{1 \perp} d \Phi_{2 \perp} \frac{2 \boldsymbol{k}_{\perp} \cdot \boldsymbol{\varepsilon}_{\perp}}{\boldsymbol{k}_{\perp}^{2}}\left(1-\mathrm{e}^{i \omega_{0} \delta z_{1}}\right), \\
& \int d \Phi_{1} d \Phi_{2} R_{3}^{(2) \mu, a} \varepsilon_{\mu}(k)=\left[\left[a, b_{2}\right], b_{1}\right]_{R}\left(b_{1}\right)_{T_{i}}\left(b_{2}\right)_{T_{j}} \int d \boldsymbol{\Phi}_{1 \perp} d \boldsymbol{\Phi}_{2 \perp} \\
& \times \frac{2\left(\boldsymbol{k}_{\perp}-\boldsymbol{q}_{1 \perp}-\boldsymbol{q}_{2 \perp}\right) \cdot \boldsymbol{\varepsilon}_{\perp}}{\left(\boldsymbol{k}_{\perp}-\boldsymbol{q}_{1 \perp}-\boldsymbol{q}_{2 \perp}\right)^{2}}\left(\mathrm{e}^{i\left(\omega_{0}-\omega_{12}\right) \delta z_{1}}-\mathrm{e}^{i \omega_{0} \delta z_{1}}\right) \mathrm{e}^{i\left(\delta z_{2}-\delta z_{1}\right)\left(\omega_{0}-\omega_{1}\right)}, \\
& \int d \Phi_{1} d \Phi_{2} R_{4}^{(2) \mu, a} \varepsilon_{\mu}(k)=\left(b_{2}\left[a, b_{1}\right]\right)_{R}\left(b_{1}\right)_{T_{i}}\left(b_{2}\right)_{T_{j}} \int d \boldsymbol{\Phi}_{1 \perp} d \boldsymbol{\Phi}_{2 \perp} \\
& \times \frac{2\left(\boldsymbol{k}_{\perp}-\boldsymbol{q}_{1 \perp}\right) \cdot \boldsymbol{\varepsilon}_{\perp}}{\left(\boldsymbol{k}_{\perp}-\boldsymbol{q}_{1 \perp}\right)^{2}}\left(\mathrm{e}^{i\left(\omega_{0}-\omega_{1}\right) \delta z_{1}}-\mathrm{e}^{i \omega_{0} \delta z_{1}}\right), \\
& \int d \Phi_{1} d \Phi_{2} R_{5}^{(2) \mu, a} \varepsilon_{\mu}(k)=\left(b_{2} a b_{1}\right)_{R}\left(b_{1}\right)_{T_{i}}\left(b_{2}\right)_{T_{j}} \int d \boldsymbol{\Phi}_{1 \perp} d \boldsymbol{\Phi}_{2 \perp} \frac{2 \boldsymbol{k}_{\perp} \cdot \boldsymbol{\varepsilon}_{\perp}}{\boldsymbol{k}_{\perp}^{2}}\left(1-\mathrm{e}^{i \omega_{0}\left(\delta z_{2}-\delta z_{1}\right)}\right) \mathrm{e}^{i \omega_{0} \delta z_{1}} \\
& \int d \Phi_{1} d \Phi_{2} R_{6}^{(2) \mu, a} \varepsilon_{\mu}(k)=\left(\left[a, b_{2}\right] b_{1}\right)_{R}\left(b_{1}\right)_{T_{i}}\left(b_{2}\right)_{T_{j}} \int d \mathbf{\Phi}_{1 \perp} d \boldsymbol{\Phi}_{2 \perp} \\
& \times \frac{2\left(\boldsymbol{k}_{\perp}-\boldsymbol{q}_{2 \perp}\right) \cdot \boldsymbol{\varepsilon}_{\perp}}{\left(\boldsymbol{k}_{\perp}-\boldsymbol{q}_{2 \perp}\right)^{2}}\left(\mathrm{e}^{-i \omega_{2}\left(\delta z_{2}-\delta z_{1}\right)}-1\right) \mathrm{e}^{i \omega_{0} \delta z_{2}},
\end{aligned}
$$

where $\omega_{0}$ and $\omega_{1}$ are defined in Eq. (6.22) above and $\omega_{2}, \omega_{12}$ are equal to:

$$
\omega_{2}=\frac{\left(\boldsymbol{k}_{\perp}-\boldsymbol{q}_{2 \perp}\right)^{2}}{x p_{0}^{+}}, \quad \omega_{12}=\frac{\left(\boldsymbol{k}_{\perp}-\boldsymbol{q}_{1 \perp}-\boldsymbol{q}_{2 \perp}\right)^{2}}{x p_{0}^{+}} .
$$

In order to understand the lowest opacity contribution to the induced bremsstrahlung, one needs to combine the single Born diagrams computed in the previous section with the contact double Born limit of the two single Born exchange diagrams. The contact limit of two single Born exchange longitudinal integrals is derived in appendix D.2. Using these results we get the following contact limits (or double Born amplitudes):

$$
\begin{aligned}
R_{1,2,3}^{(2 c)} & =\frac{1}{2} R_{1,2,3}^{(2)}\left(\delta z_{2}=\delta z_{1}\right), \\
R_{4}^{(2 c)} & =R_{4}^{(2)}\left(\delta z_{2}=\delta z_{1}\right), \\
R_{5,6}^{(2 c)} & =0 .
\end{aligned}
$$

All results in this and the previous subsections, derived in the framework of $\mathrm{SCET}_{\mathrm{G}}$, agree with the soft gluon approximation previously derived in the literature [40, 51]. For example, to first order in opacity and without explicitly showing the integral over the position of the scattering center we find:

$$
k^{+} \frac{d N^{g}(F S)}{d k^{+} d^{2} \mathbf{k}_{\perp}}=\left(\frac{N}{A_{\perp}}\right) \frac{C_{F} \alpha_{s}}{\pi^{2}} \int d^{2} \boldsymbol{q}_{\perp}\left[\frac{d \sigma_{\mathrm{el}}(R, T)}{d^{2} \boldsymbol{q}_{\perp}}\right]\left(\frac{2 \boldsymbol{k}_{\perp} \cdot \boldsymbol{q}_{\perp}}{\boldsymbol{k}_{\perp}^{2}\left(\boldsymbol{k}_{\perp}-\boldsymbol{q}_{\perp}\right)^{2}}\right)\left(1-\cos \left[\frac{\left(\boldsymbol{k}_{\perp}-\boldsymbol{q}_{\perp}\right)^{2}}{k^{+}} \delta z\right]\right) .
$$

Note that in the equation above the superscript "g" stands for the radiated gluons and should not be confused with Glauber gluons, denoted by "G". However, we can go beyond that and calculate the finite- $x$ corrections to single and double Born diagrams, similarly to the full Altarelli-Parisi splitting kernel, and not just its soft gluon limit. In section 9 below we derive analytical formulas for these finite- $x$ corrections to radiative energy loss at first order in opacity. 


\section{Gauge invariance of the jet broadening and the medium-induced bremsstrahlung results}

In this section we demonstrate that the single and double Born amplitudes calculated in the previous two sections are gauge invariant. As it is known on the example of SCET, the gauge structure of effective theory is more rich than that of a full theory. This is a simple consequence of having multiple modes for the gauge field. In our calculation we deal with two types of gluons: collinear and Glauber. Thus, we can gauge fix these two modes completely independently without changing any physical result. Since Glauber mode is an off-shell mode, it is integrated out from the theory and is presented in the form of the potential term in Eq. (4.15). Thus, the only gauge freedom for Glauber gluons is the choice of the propagator $\Delta_{\mu \nu}(q)$ in our effective potential, which in principle can be arbitrary. The collinear gluon field on the other hand is a truly propagating degree of freedom, with the corresponding kinetic term contained in the SCET Lagrangian. For each collinear gluon one could choose a certain gauge-fixing term.

In the previous two sections we considered the fully covariant gauge, in the sense that both collinear gluons are quantized in the covariant gauge, and also for the Glauber Lagrangian we choose covariant gluon propagator $\Delta_{\mu \nu}(q)_{R_{\xi}}$. Below we consider two alternative gauge choices and demonstrate there equivalence to the previous results. First, we consider a hybrid gauge where the collinear gluons are in the positive light-cone gauge and the Glauber potential is in the covariant gauge. Second, we choose both the collinear gluons and the Glauber potential term in the positive light-cone gauge $A_{c, g}^{+}=0$.

The equivalence of all considered cases can be formulated in the following way. All the diagrams in consideration are some combination of elastic scattering amplitudes with a real gluon emission amplitude. Since each of these two processes is gauge-independent, the resulting amplitudes, when contracted with the physical external gluon polarization vectors, are the same even when each of the gauges are chosen independently. This is equivalent to the statement that different modes for the gauge field in the effective theory can be gauge-fixed independently.

\subsection{Hybrid gauge $A_{c}^{+}=0, R_{\xi}\left(A_{g}\right)$}

We start from the hybrid gauge because it is simpler from a practical point of view. In this case we consider the light-cone gauge for the collinear gluons, while the Glauber propagator in the potential is taken to be in the covariant gauge. The Feynman rules for this gauge are contained in figure 11. Note that the collinear Wilson line $W_{n}=1$ is absent in this case. For example, for the single Born radiative energy loss case we only have the first three diagrams compared to the covariant gauge case in figure 5, while the remaining two are absent. Since the collinear gluons are in the light-cone gauge, the transverse gauge link at infinity could in principle add new Feynman rules, where Glauber gluons arise from the transverse Wilson line $T_{n}$ (see appendix E for a brief review of this subject). However, in this case it doesn't generate such a Feynman rule for the following reason. The diagram for which the $T_{n}$ Wilson line generates a Glauber gluon that couples to the source vanishes in this hybrid gauge because the propagator of Glauber gluon

doted with the transverse gauge field and the source term vanishes: $A_{\perp}^{i} g^{i \mu} v_{\mu}=0$. However as we will see from the next subsection this is not the case in the fully positive light-cone gauge, where this interaction plays an important role. This is another reason why the hybrid gauge is so convenient.

For brevity, we consider explicitly only single Born diagrams for the radiative energy loss in detail and then quote results for the remaining cases. We have only the first three diagrams in figure 5 for this case. 
Using the Feynman rules from the appendix C we obtain:

$$
\begin{aligned}
\left(R_{1}^{\mu, a}\right)_{\mathrm{hyb}}= & i(a)_{R}\left(n^{\mu}+\frac{\gamma_{\perp}^{\mu}\left(\not p_{\perp}+\not k_{\perp}\right)}{p^{+}+k^{+}}+\frac{\not p_{\perp} \gamma_{\perp}^{\mu}}{p^{+}}\right) \frac{i\left(p^{+}+k^{+}\right)}{(p+k)^{2}+i \varepsilon} i\left(b_{1}\right)_{R}\left(b_{1}\right)_{T_{i}} i \Delta_{g}\left(p+k, q_{1}\right) \\
\left(R_{2}^{\mu, a}\right)_{\mathrm{hyb}}= & i\left(b_{1}\right)_{R}\left(b_{1}\right)_{T_{i}} i \Delta_{g}\left(p, q_{1}\right) i(a)_{R}\left(n^{\mu}+\frac{\gamma_{\perp}^{\mu}\left(\not p_{\perp}-\not q_{1}\right)}{p^{+}}+\frac{\not p_{\perp} \gamma_{\perp}^{\mu}}{p^{+}}\right) i \Delta_{g}\left(p+k, q_{1}\right) \\
\left(R_{3}^{\mu, a}\right)_{\mathrm{hyb}}= & i\left(c_{1}\right)_{R}\left(n^{\rho_{1}}+\frac{\gamma_{\perp}^{\rho_{1}}\left(\not p_{\perp}+\not k_{\perp}-\not \phi_{1}\right)}{p^{+}}+\frac{\not p_{\perp} \gamma_{\perp}^{\rho_{1}}}{p^{+}}\right) \frac{(-i) \Delta_{g}\left(k, q_{1}\right)}{\bar{n} \cdot k} N_{\rho_{1} \rho_{2}}^{(\mathrm{hyb})}\left(k-q_{1}\right) i \Delta_{g}\left(p+k, q_{1}\right) \\
& \times g_{\perp}^{\mu \rho_{2}} f^{c_{1} a b_{1}}\left(b_{1}\right)_{T_{i}} \bar{n} \cdot k .
\end{aligned}
$$

While first two amplitudes immediately match those in the covariant gauge given in Eq. (6.14), Eq. (6.15), in order to work out the third amplitude, the numerator $N_{\mu \nu}^{\text {hyb }}$ of the positive light-cone gauge gluon propagator should be inserted. The following identity is straightforward to verify using the Feynman rules at hand:

$$
\varepsilon_{\nu}(k) g_{\perp}^{\rho \nu} \bar{n} \cdot k N_{\mu \rho}^{(\mathrm{hyb})}\left(k-q_{1}\right)=\varepsilon_{\nu}(k) \tilde{\Sigma}_{1}^{\rho \nu}\left(k-q_{1}, k\right) N_{\mu \rho}^{\left(R_{\xi}\right)}\left(k-q_{1}\right) .
$$

Thus, we arrive to the result that $\left(R_{1,2,3}^{\mu, a}\right)_{\text {hyb }} \equiv\left(R_{1,2,3}^{\mu, a}\right)_{R_{\xi}}$. The remaining two diagrams in the figure 5 are zero in the covariant gauge ${ }^{13}$ and are simply absent in the hybrid gauge.

Similarly, proceeding with the remaining single and double Born amplitudes for the broadening and radiation we verify that the hybrid gauge results match the covariant gauge results at the amplitude level, as they should:

$$
\begin{gathered}
\left(B^{(1) q, g}\right)_{\text {hyb }}=\left(B^{(1) q, g}\right)_{R_{\xi}}, \quad\left(B^{(2 c) q, g}\right)_{\mathrm{hyb}}=\left(B^{(2 c) q, g}\right)_{R_{\xi}}, \\
\left(R^{(1)}\right)_{\mathrm{hyb}}=\left(R^{(1)}\right)_{R_{\xi}}, \quad\left(R^{(2 c)}\right)_{\mathrm{hyb}}=\left(R^{(2 c)}\right)_{R_{\xi}} .
\end{gathered}
$$

\subsection{Positive light-cone gauge $A_{c, g}^{+}=0$}

We consider the positive light-cone gauge in this subsection. The collinear gluons are treated in the lightcone gauge as well as the Glauber gluons. Similarly to the previous subsection, we consider in details the single Born diagrams for radiative energy loss and later on quote the result in all other cases. Clearly, the collinear Wilson line vanishes again in this gauge $W_{n}=1$. However, the transverse gauge link (see appendix E) gives a new non-trivial Feynman diagram. In figure 7 we show the two new diagrams that appear in our gauge choice. The first one directly follows from the collinear part of the Lagrangian of SCET, once the background field with the light-cone vector potential scaling is added. It is summarized in figure 11 of appendix $\mathrm{C}$. The second diagram in figure 7 arises from the $T_{n}$ Wilson line emitting a Glauber gluon which interacts with the source. The corresponding Feynman rule is derived in the appendix E and is summarized in figure 14. As one can see from this Feynman rule, it depends on the light-cone prescription. So does the light-cone (Glauber) gluon propagator. We will see in this section that these two dependences

\footnotetext{
${ }^{13}$ This is true only for the same choice of physical polarization vectors in the covariant, light-cone and hybrid gauge calculations. If one chooses arbitrary polarization vectors, once squared and summed over polarizations the result is independent on them. However, in this case it would be impossible to verify the gauge invariance with the hybrid or positive light-cone gauge, at the amplitude level.
} 

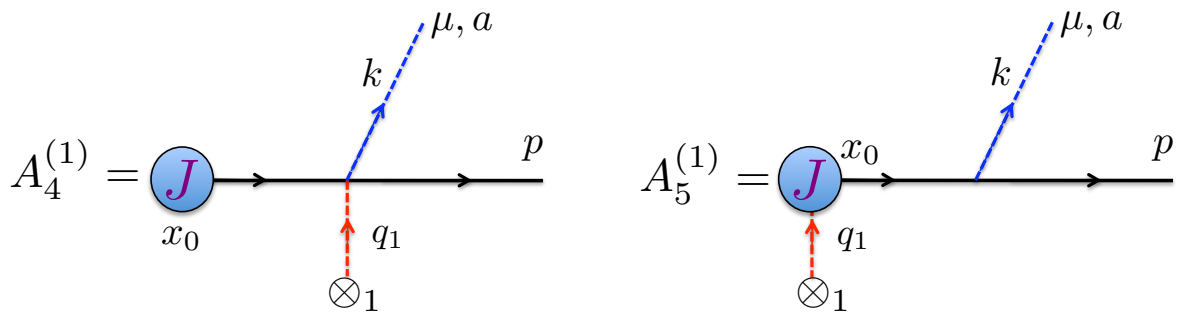

Figure 7: Two additional diagrams that appear in the light-cone gauge for the single Born amplitude for radiative energy loss. Left: term arising from the $\mathrm{SCET}_{\mathrm{G}}$ Lagrangian in the light-cone gauge. Right: $T_{n}$ Wilson line contribution to the matrix element. The notation for the scattering centers is the following: $\otimes_{1}=\left[x_{1}, q_{1},\left(b_{1}\right)_{i}\right]$.

cancel non-trivially and the final answer in this case is identical to the covariant gauge calculation above for all light-cone prescriptions. Similar cancellation was found in [54] by introducing the $T$ Wilson line to SCET and calculating the jet function at one-loop in the light-cone gauge.

Using the Feynman rules of this gauge we evaluate the first three diagrams in figure 5, which are present in this gauge as well:

$$
\left(R_{1,2,3}^{(1) \mu, a}\right)_{A^{+}}=\left(R_{1,2,3}^{(1) \mu, a}\right)_{R_{\xi}}+\Delta R_{1,2,3}^{(1) \mu, a}
$$

where the correction terms $\Delta R_{i}^{(1) \mu, a}$ sum up to the following expression:

$$
\begin{gathered}
\int d \Phi_{1}\left(\Delta R_{1}^{(1) \mu, a}+\Delta R_{2}^{(1) \mu, a}+\Delta R_{3}^{(1) \mu, a}\right)=\int d \Phi_{1 \perp}\left[\left(b_{1} a\right)_{R}\left(b_{1}\right)_{T_{i}}\left(-\not \phi_{1 \perp} \gamma_{\perp}^{\mu} \frac{p^{+}+k^{+}}{p^{+}} \Delta I\right)\right. \\
\left.+\left(a b_{1}\right)_{R}\left(b_{1}\right)_{T_{i}}\left(-\gamma_{\perp}^{\mu} \not q_{1 \perp} \Delta I+\frac{p^{+}+k^{+}}{(p+k)^{2}} \Delta_{A^{+}}\left(\frac{2 \boldsymbol{k}_{\perp}^{\mu}}{k^{+}}+\frac{\gamma_{\perp}^{\mu}\left(\not p_{\perp}+\not k_{\perp}\right)}{p^{+}+k^{+}}+\frac{\not p_{\perp} \gamma_{\perp}^{\mu}}{p^{+}}\right)\right)\right] .
\end{gathered}
$$

The two longitudinal integrals $\Delta I$ and $\Delta_{A^{+}}$are defined in the following way ${ }^{14}$ :

$$
\begin{aligned}
& \Delta I=\int \frac{d q_{1}^{-}}{2 \pi} \mathrm{e}^{i q_{1}^{-} \Delta z_{1}} \frac{1}{\left(p+k-q_{1}\right)^{2}} \frac{1}{\left[q_{1}^{+}\right]}, \\
& \Delta_{A^{+}}=\int \frac{d q_{1}^{-}}{2 \pi} \mathrm{e}^{i q_{1}^{-} \delta z_{1}} \frac{1}{\left[q_{1}^{+}\right]} .
\end{aligned}
$$

The second integral is summarized in the table 7.2 and vanishes in the $-i \varepsilon$ light-cone prescription. However, the first integral is clearly non-zero in any of the prescriptions, since its value comes from the poles of the propagator denominator. Luckily the first diagram in figure 7 completely cancels this term:

$$
\int d \Phi_{1}\left(R_{4}^{(1)}\right)_{A^{+}}^{\mu, a}=\int d \Phi_{1 \perp}\left[\left(b_{1} a\right)_{R}\left(b_{1}\right)_{T_{i}} \phi_{1 \perp} \gamma_{\perp}^{\mu} \frac{p^{+}+k^{+}}{p^{+}} \Delta I+\left(a b_{1}\right)_{R}\left(b_{1}\right)_{T_{i}} \gamma_{\perp}^{\mu} \phi_{1 \perp} \Delta I\right] .
$$

When the amplitude $R_{4}^{(1)}$ is added to the first three diagrams, the $\Delta I$ integral vanishes as expected, while the $\Delta_{A^{+}}$integrals stays. It vanishes only in the $-i \varepsilon$ light-cone prescription, exactly in which the transverse gauge link vanishes according to Feynman rule derived in appendix E. In all other prescriptions,

\footnotetext{
${ }^{14}$ Note that $q_{1}^{+} \equiv-q_{1}^{-}$in this equations, set by the $\delta\left(q_{1}^{0}\right)$ in $v\left(q_{1}\right)$.
} 


\begin{tabular}{||c||c||c|}
\hline Prescription & $\frac{1}{\left[k^{+}\right]}$ & $i \cdot \Delta_{A^{+}}$ \\
\hline \hline$+i \varepsilon$ & $\frac{1}{k^{+}+i \varepsilon}$ & 1 \\
$-i \varepsilon$ & $\frac{1}{k^{+}-i \varepsilon}$ & 0 \\
PV & $\frac{1}{2}\left(\frac{1}{k^{+}+i \varepsilon}+\frac{1}{k^{+}-i \varepsilon}\right)$ & $\frac{1}{2}$ \\
ML & $\frac{1}{k^{+}+i \varepsilon \operatorname{sign}\left(k^{-}\right)}$ & $\frac{1}{2}$ \\
\hline
\end{tabular}

Table 2: Prescription dependent integral appearing in the light-cone gauge.

the contribution from the transverse Wilson line does not vanish and we include it by calculating the second amplitude in figure 7. We explicitly see that by including these Feynman diagram the prescription dependence cancels in all three remaining cases: $+i \varepsilon, \mathrm{PV}, \mathrm{ML}$, which happens through pushing the $q^{-}$ pole into the negative complex plane which leads to the zero integral. Thus, by including the $T$-Wilson line into the calculation we get the same result in light-cone gauge as in the covariant gauge for all light-cone prescriptions. To see this explicitly we apply Feynman rule for the single Glauber gluon exchange from quark $T$ - Wilson line, using appendix E (see figure 14) and obtain:

$$
\int d \Phi_{1}\left(R_{5}^{(1)}\right)_{A^{+}}^{\mu, a}=\left(a b_{1}\right)_{R}\left(b_{1}\right)_{i} \int d \Phi_{1 \perp} i\left[n^{\mu}+\frac{\left(\not p_{\perp}+\not k_{\perp}\right) \gamma_{\perp}^{\mu}}{p^{+}+k^{+}}+\frac{\gamma_{\perp}^{\mu} \not p_{\perp}}{p^{+}}\right] i \frac{\left(p^{+}+k^{+}\right)}{(p+k)^{2}} \Delta_{T},
$$

where the longitudinal integral $\Delta_{T}$ is defined according to:

$$
\Delta_{T}=C_{\infty}^{\text {Pres }} \int \frac{d q_{1}^{-}}{2 \pi} \mathrm{e}^{i q_{1}^{-} \delta z_{1}}\left(\frac{1}{q_{1}^{+}+i \varepsilon}-\frac{1}{q_{1}^{+}-i \varepsilon}\right) .
$$

Using table $\mathrm{E}$ for the prescription dependence of $C_{\infty}^{\text {Pres }}$ we verify that it vanishes in the $-i \varepsilon$ prescription ${ }^{15}$ and in all other prescriptions makes the $\Delta_{T}$ integral cancel exactly the contribution from $\Delta_{A^{+}}$integral above. Thus, in the presence of a transverse gauge link we verify the gauge invariance for all prescriptions:

$$
\int d \Phi_{1}\left(\Delta R_{1}^{(1) \mu, a}+\Delta R_{2}^{(1) \mu, a}+\Delta R_{3}^{(1) \mu, a}+R_{4}^{(1) \mu, a}+R_{5}^{(1) \mu, a}\right)=0 .
$$

Calculations in all other cases, like jet broadening and radiative energy loss in single and double Born amplitudes, are similar to case considered above. We quote the results:

$$
\begin{gathered}
\left(B^{(1) q, g}\right)_{A^{+}}=\left(B^{(1) q, g}\right)_{R_{\xi}}, \quad\left(B_{q, g}^{(2 c)}\right)_{A^{+}}=\left(B_{q, g}^{(2 c)}\right)_{R_{\xi}}, \\
\left(R^{(1)}\right)_{A^{+}}=\left(R^{(1)}\right)_{R_{\xi}}, \quad\left(R^{(2 c)}\right)_{A^{+}}=\left(R^{(2 c)}\right)_{R_{\xi}} .
\end{gathered}
$$

Thus, for all light-cone prescriptions we get an unambiguous result, same as in the covariant gauge. If one ignores the transverse gauge link, one should employ one particular prescription, $-i \varepsilon$ in this case, in order to recover the correct result. For practical purposes this gauge choice is the most difficult from all three that we considered in this paper.

\footnotetext{
${ }^{15}$ Note that in the $-i \varepsilon$ prescription the integral $\Delta_{A^{+}}$vanishes as well.
} 


\section{Identifying the in-medium jet evolution kernels}

In the preceding sections we calculated the amplitudes for collisional and radiative jet interaction at a particular position $x_{i}$ and demonstrated the gauge invariance of the end results. It is clear that a direct calculation of all diagrams for any number of scattering positions $x_{i_{1}}, x_{i_{2}}, \cdots$ is not possible. From the results at hand, however, we can deduce the effect of an in-medium interaction at the amplitude and cross section levels in momentum space and employ the resulting kernels to describe a number of collisional and radiative processes in cold and hot nuclear matter as solutions to algebraic recurrence relations with suitably chosen initial conditions $[40,37,38,51,55,56]$.

In the absence of long-range color correlations in the target, the relevant interactions at position $i_{n}$ to lowest order in $\alpha_{s}$ that can build up to a jet-medium cross sections (a total of two Glauber exchanges in the forward cut diagram) can be expresses as follows:

$$
\begin{aligned}
\mathcal{A}_{i_{1} \cdots i_{n-1}, 0}(\alpha) & \equiv \hat{I} \mathcal{A}_{i_{1} \cdots i_{n-1}}(\alpha), \\
\mathcal{A}_{i_{1} \cdots i_{n-1}, 1}(\alpha) & \equiv \hat{D} \mathcal{A}_{i_{1} \cdots i_{n-1}}(\alpha), \\
\mathcal{A}_{i_{1} \cdots i_{n-1}, 2}(\alpha) & \equiv \hat{V} \mathcal{A}_{i_{1} \cdots i_{n-1}}(\alpha) .
\end{aligned}
$$

Here, $\alpha$ represents a set of relevant quantum numbers, such as longitudinal momentum, transverse momentum and color. The kinematic and color structure of the scattering is contained in the unit $(\hat{I})$, direct $(\hat{D})$ and virtual $(\hat{V})$ operators, which evolve the amplitude $\mathcal{A}_{i_{1} \cdots i_{n-1}}(\alpha)$ of the propagating system. The unit operator indicates that there are no Glauber gluon exchanges between the projectile and the target at position $x_{i_{n}}\left(i_{n}=0\right)$. The direct operator indicates a single Glauber gluon exchange between the projectile and the target at position $x_{i_{n}}\left(i_{n}=1\right)$. The virtual operator indicates two Glauber gluon exchanges between the projectile and the target at position $x_{i_{n}}\left(i_{n}=2\right)$, i.e. in the contact limit. The set of amplitude indices in Eqs. (8.1)-(8.3), thus, encodes the complete history of jet interactions in QCD matter. Repeating the basic operator steps in Eqs. (8.1)-(8.3) any amplitude that includes parton scatterings inside the medium can be iteratively derived from the unperturbed jet production amplitude

$$
\mathcal{A}_{i_{1} \cdots i_{n}}(\alpha)=\prod_{m=1}^{n}\left[\delta_{0, i_{m}}+\delta_{1, i_{m}} \hat{D}_{m}+\delta_{2, i_{m}} \hat{V}_{m}\right] J_{0}(\alpha) \text {. }
$$

Time ordering is implicit in the above formula. The amplitudes $\overline{\mathcal{A}}^{i_{1} \cdots i_{n}}(p, c)$ are the complementary amplitudes to $\mathcal{A}_{i_{1} \cdots i_{n}}(\alpha)$ given by

$$
\overline{\mathcal{A}}^{i_{1} \cdots i_{n}}(\alpha) \equiv J_{0}^{\dagger}(\alpha) \prod_{m=1}^{n}\left[\delta_{0, i_{m}} \hat{V}_{m}^{\dagger}+\delta_{1, i_{m}} \hat{D}_{m}^{\dagger}+\delta_{2, i_{m}}\right] .
$$

The differential jet or radiative gluon distribution, depending on the problem, can be expresses as a sum over the interactions in the medium $d N(\alpha) / d P S=\sum_{n=0}^{\infty} d N^{(n)}(\alpha) / d P S$. Each contribution with a fixed number of interactions $n$ can, in turn be represented as

$$
d N^{(n)}(\alpha) / d P S \propto \overline{\mathcal{A}}^{i_{1} \cdots i_{n}}(\alpha) \mathcal{A}_{i_{1} \cdots i_{n}}(\alpha) \propto \operatorname{Tr} \sum_{i_{1}=0}^{2} \cdots \sum_{i_{n}=0}^{2} \overline{\mathcal{A}}^{i_{1} \cdots i_{n}}(\alpha) A_{i_{1} \cdots i_{n}}(\alpha) .
$$

The trace is over any uncontracted color and spin/polarization indices. Using Eqs. (8.4), (8.5) we obtain a simple recursion identity which relates $d N^{(n)} / d P S$ to $d N^{(n-1)} / d P S$ through the reaction operator $\hat{R}$

$$
d N^{(n)} / d P S \propto \overline{\mathcal{A}}^{i_{1} \cdots i_{n-1}} \hat{R}_{n} \mathcal{A}_{i_{1} \cdots i_{n-1}}, \quad \hat{R}_{n}=\hat{D}_{n}^{\dagger} \hat{D}_{n}+\hat{V}_{n}+\hat{V}_{n}^{\dagger} .
$$


Thus, the most important step in investigating the effects of the medium on jet propagation is the identification of the reaction operator $\hat{R}$. We note that in problems that involve long-range coherence effects, such as radiative gluon re-interactions in the QCD matter, the recurrence relations may be inhomogeneous.

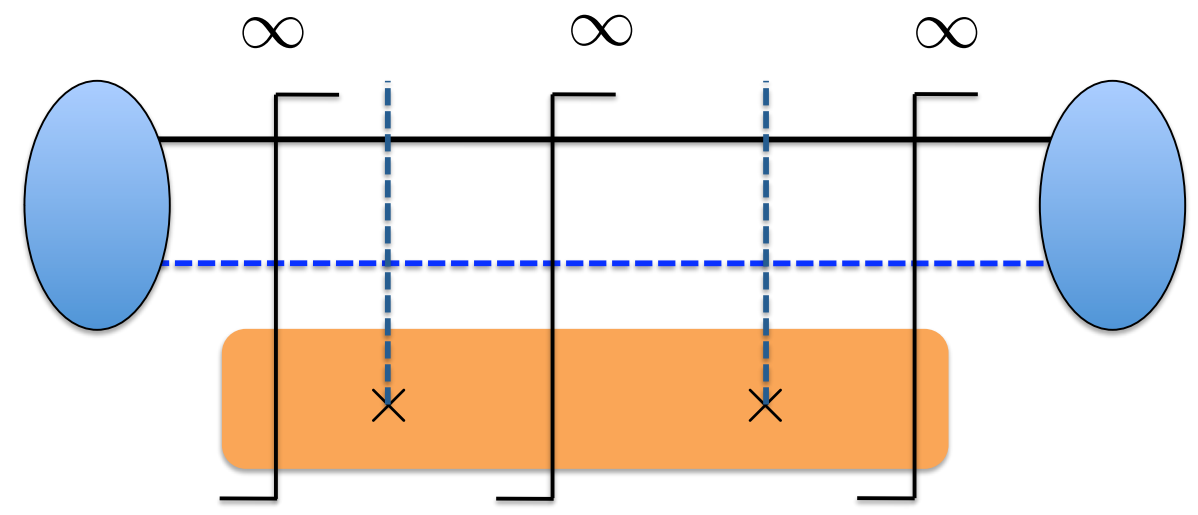

Figure 8: Three contributions to the cross section, associated with the application of the reaction operator, correspond to the three $t=\infty$ cuts.

\subsection{Reaction operator for collisional interactions}

With the direct and virtual interactions of a jet calculated in section 5 , we can identify the form of the reaction operator for collisional interactions acting on a jet of momentum $p$

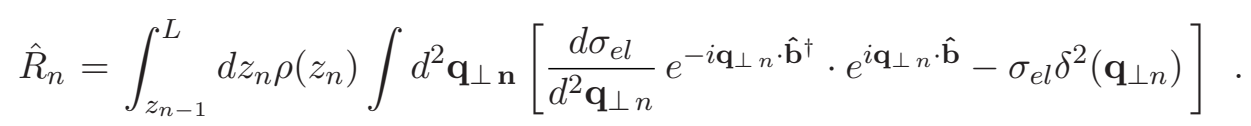

Here, $L$ is the thickness of the target, $\hat{\mathbf{b}}=i \vec{\nabla}_{\mathbf{p}_{\perp}}$ is the 2-dimensional impact parameter operator conjugate to the transverse momentum $\mathbf{p}$ acting to the right and $\hat{\mathbf{b}}^{\dagger}=-i \overleftarrow{\nabla}_{\mathbf{p}_{\perp}}$ is its Hermitian conjugate acting to the left. In this paper we explicitly proved the gauge invariance of this reaction operator. Our results are not surprising since $\hat{R}$ is expressed in terms of physical quantities, such as cross sections and densities and kinematic modification to the initial jet distribution.

The contribution to the jet transverse momentum distribution from $n$ medium interactions can be written as:

$$
\frac{d N^{(n)}\left(\mathbf{p}_{\perp}\right)}{d^{2} \mathbf{p}_{\perp}}=\prod_{i=1}^{n} \int_{z_{i-1}}^{L} \frac{d z_{i}}{\lambda} \int d^{2} \mathbf{q}_{\perp i}\left[\frac{1}{\sigma_{e l}\left(z_{i}\right)} \frac{d \sigma_{e l}\left(z_{i}\right)}{d^{2} \mathbf{q}_{\perp i}}\left(e^{-\mathbf{q}_{\perp i} \cdot \vec{\nabla}_{\mathbf{p}_{\perp}}}\right)-\delta^{2}\left(\mathbf{q}_{\perp i}\right)\right] \frac{d N^{(0)}\left(\mathbf{p}_{\perp}\right)}{d^{2} \mathbf{p}_{\perp}}
$$

where $\lambda$ is the mean free path of the jet in the medium. Summing over all fixed- $n$ contributions that Eq. (8.9) specifies, we obtain the most general result for the broadening of jets that propagate and interact in strongly-interacting matter.

Simplifications of the sum over the contributions given by Eq. (8.9) can be obtained for special cases. For example, for a uniform density the integrals along the path of the jet can be performed immediately. With the notation $\chi=L / \lambda$, the result reads:

$$
\frac{d N\left(\mathbf{p}_{\perp}\right)}{d^{2} \mathbf{p}_{\perp}}=\sum_{n=0}^{\infty} \frac{d N^{(n)}\left(\mathbf{p}_{\perp}\right)}{d^{2} \mathbf{p}_{\perp}}=\sum_{n=0}^{\infty} e^{-\chi} \frac{\chi^{n}}{n !} \int \prod_{i=1}^{n} d^{2} \mathbf{q}_{i} \frac{1}{\sigma_{e l}} \frac{d \sigma_{e l}}{d^{2} \mathbf{q}_{\perp i}} d N^{(0)}\left(\mathbf{p}_{\perp}-\mathbf{q}_{\perp 1}-\cdots-\mathbf{q}_{\perp n}\right)
$$


This is the partonic version of the Glauber multiple collision series where the jet interaction in the medium are described by a Poisson distribution and the momentum distribution is modified by the normalized differential scattering cross section [37, 38].

A final simplification can be achieved if one recognizes that the individual jet-medium interactions are of finite range $r=1 / \mu$, such as the one discussed in sections 3 and 4 . Let us take the initial jet distribution to be in the positive light-cone direction, $d N\left(\mathbf{p}_{\perp}\right) / d^{2} \mathbf{p}_{\perp}=\delta^{2}\left(\mathbf{p}_{\perp}\right)$. The Gaussian approximation can be best understood in impact parameter space where the Fourier transform of the normalized scattering cross section reads

$$
\frac{d \tilde{\sigma}_{e l}}{d^{2} \mathbf{q}_{\perp}}(\mathbf{b})=\int \frac{d^{2} \mathbf{q}_{\perp}}{(2 \pi)^{2}} e^{-i \mathbf{q}_{\perp} \cdot \mathbf{b}} \frac{1}{\pi} \frac{\mu^{2}}{\left(\mathbf{q}_{\perp}^{2}+\mu^{2}\right)^{2}}=\frac{\mu b}{4 \pi^{2}} K_{1}(\mu b) \approx \frac{1}{4 \pi^{2}}\left(1-\frac{\xi \mu^{2} b^{2}}{2}+\mathcal{O}\left(b^{3}\right)\right) .
$$

Here $b=|\mathbf{b}|$ and in the quadratic term in Eq. (8.11) the $\log 2 /(1.08 \mu b)$ multiplicative factor has been absorbed into an approximately $b$-independent constant $\xi$. Fourier transforming back to momentum space we obtain:

$$
\frac{d N\left(\mathbf{p}_{\perp}\right)}{d^{2} \mathbf{p}_{\perp}}=\int d^{2} \mathbf{b} e^{i \mathbf{p}_{\perp} \cdot \mathbf{b}} \frac{1}{(2 \pi)^{2}} e^{-\frac{\chi \mu^{2} \xi b^{2}}{2}}=\frac{1}{2 \pi} \frac{e^{-\frac{p_{\perp}^{2}}{2 \chi \mu^{2} \xi}}}{\chi \mu^{2} \xi} .
$$

The resulting distribution is of two dimensional Gaussian form and has a width of $2 \chi \mu^{2} \xi$, i.e. $\left\langle\mathbf{p}_{\perp}^{2}\right\rangle=$ $2 \chi \mu^{2} \xi$. It should be noted that the Gaussian approximation Eq. (8.12) is only applicable for small transverse momenta. It misses the Rutherford scattering power law $1 / \mathbf{p}_{\perp}^{4}$ behavior for $\mathbf{p}_{\perp}^{2} \geq 2 \chi \mu^{2}$. The general solution the problem of jet multiple scattering is given by Eq. (8.9) [37].

\subsection{Reaction operator for medium-induced gluon bremsstrahlung}

The derivation of the reaction operator for radiative processes is significantly more complicated in comparison to the one for collisional interactions. The first step is to reorganize the amplitudes obtained in section 6 and to identify the contributions that can be interpreted as an interaction of the parent parton, an interaction of the already radiated gluon $\left(\hat{S}_{n}\right)$ and, finally, a genuinely new source of radiation associated with this particular scattering $\left(\hat{B}_{n}\right)$.

We introduce the following notation for the radiative gluon inverse formation times:

$$
\omega_{0}=\frac{\mathbf{k}_{\perp}^{2}}{2 \omega}, \omega_{i}=\frac{\left(\mathbf{k}_{\perp}-\mathbf{q}_{\perp i}\right)^{2}}{k^{+}}, \omega_{(i j)}=\frac{\left(\mathbf{k}_{\perp}-\mathbf{q}_{\perp i}-\mathbf{q}_{\perp j}\right)^{2}}{k^{+}}, \omega_{(i \cdots j)}=\frac{\left(\mathbf{k}_{\perp}-\sum_{m=i}^{j} \mathbf{q}_{\perp m}\right)^{2}}{k^{+}},
$$

and transverse momentum propagators:

$$
\begin{array}{rlrl}
\mathbf{H} & =\frac{\mathbf{k}_{\perp}}{\mathbf{k}_{\perp}^{2}}, & \mathbf{C}_{\left(i_{1} i_{2} \cdots i_{m}\right)}=\frac{\mathbf{k}_{\perp}-\mathbf{q}_{\perp i_{1}}-\mathbf{q}_{\perp i_{2}}-\cdots-\mathbf{q}_{\perp i_{m}}}{\left(\mathbf{k}_{\perp}-\mathbf{q}_{\perp i_{1}}-\mathbf{q}_{\perp i_{2}}-\cdots-\mathbf{q}_{\perp i_{m}}\right)^{2}}, \\
\mathbf{B}_{i}=\mathbf{H}-\mathbf{C}_{i}, & \mathbf{B}_{\left(i_{1} i_{2} \cdots i_{m}\right)\left(j_{1} j_{2} \cdots j_{n}\right)}=\mathbf{C}_{\left(i_{1} i_{2} \cdots i_{m}\right)}-\mathbf{C}_{\left(j_{1} j_{2} \cdots j_{n}\right)} .
\end{array}
$$

Further, we recall that each double Born exchange yields a factor $-1 / 2$ and the numbers of these exchanges in the amplitude and its complementary are:

$$
N_{v}=N_{v}\left(\mathcal{A}_{i_{1} \cdots i_{n-1}}\right)=\sum_{m=1}^{n-1} \delta_{2, i_{m}}, \quad \bar{N}_{v}=N_{v}\left(\overline{\mathcal{A}}^{i_{1} \cdots i_{n-1}}\right)=\sum_{m=1}^{n-1} \delta_{0, i_{m}} .
$$

Finally, the color matrices associated with the collisional interactions of the jet are denoted by

$$
T_{e l}\left(\mathcal{A}_{i_{1} \cdots i_{n-1}}\right) \equiv\left(a_{n-1}\right)^{i_{n-1}} \cdots\left(a_{1}\right)^{i_{1}}, \quad T_{e l}^{\dagger}\left(\overline{\mathcal{A}}^{i_{1} \cdots i_{n-1}}\right) \equiv\left(a_{1}\right)^{2-i_{1}} \cdots\left(a_{n-1}\right)^{2-i_{n-1}} .
$$


We first examine the single Glauber exchange and the direct operator reads:

$$
\begin{aligned}
\hat{D}_{n} & \equiv\left(a_{n}+\hat{S}_{n}+\hat{B}_{n}\right) \\
& =a_{n}+e^{i\left(\omega_{0}-\omega_{n}\right) z_{n}} e^{i \mathbf{q}_{n} \cdot \hat{\mathbf{b}}} \times i f^{c a_{n} d}-\left(-\frac{1}{2}\right)^{N_{v}\left(\mathcal{A}_{i_{1} \cdots i_{n-1}}\right)} \mathbf{B}_{n} e^{i \omega_{0} z_{n}}\left[c, a_{n}\right] T_{e l}\left(\mathcal{A}_{i_{1} \cdots i_{n-1}}\right) .
\end{aligned}
$$

We note that the common factor to all diagrams $2 i g \epsilon \cdot(\cdots)$, where the transverse polarization vector $\boldsymbol{\epsilon}$ contracts with the 2D propagators in Eq. (8.14), is not explicitly shown for brevity. It is understood in Eq. (8.17) that $i f^{c a_{n} d}$ rotates the color of the radiated gluon $d$ : if $f^{c a_{n} d} d=\left[c, a_{n}\right]$. Next, we identify the virtual operator:

$$
\begin{aligned}
\hat{V}_{n} & \equiv-\frac{1}{2}\left(C_{A}+C_{R}\right)-a_{n} \hat{S}_{n}-a_{n} \hat{B}_{n}=-a_{n} \hat{D}_{n}-\frac{1}{2}\left(C_{A}-C_{R}\right) \\
& =-\frac{C_{R}+C_{A}}{2}-e^{i\left(\omega_{0}-\omega_{n}\right) z_{n}} e^{i \mathbf{q}_{n} \cdot \hat{\mathbf{b}}} a_{n} i f^{c a_{n} d}-\left(-\frac{1}{2}\right)^{N_{v}\left(\mathcal{A}_{i_{1} \cdots i_{n-1}}\right)} \frac{C_{A}}{2} \mathbf{B}_{n} e^{i \omega_{0} z_{n}} c a_{n-1}^{i_{n-1}} \cdots a_{1}^{i_{1}} .
\end{aligned}
$$

Substituting Eqs. (8.17) and (8.18) in the definition of the reaction operator we find:

$$
\begin{aligned}
\hat{R}_{n} & =\left(\hat{D}_{n}-a_{n}\right)^{\dagger}\left(\hat{D}_{n}-a_{n}\right)-C_{A}=\left(\hat{S}_{n}+\hat{B}_{n}\right)^{\dagger}\left(\hat{S}_{n}+\hat{B}_{n}\right)-C_{A} \\
& =C_{A}\left(e^{-\mathbf{q}_{n} \overleftarrow{\nabla_{\mathbf{k}}}} e^{-\mathbf{q}_{n} \overrightarrow{\nabla_{\mathbf{k}}}}-1\right)-2 C_{A} \mathbf{B}_{n} \cdot\left(\boldsymbol{R e} e^{-i \omega_{n} z_{n}} e^{i \mathbf{q}_{\mathbf{n}} \cdot \hat{\mathbf{b}}} \mathbf{I}_{n-1}\right)+\delta_{n, 1} C_{A} C_{R}\left|\mathbf{B}_{1}\right|^{2}
\end{aligned}
$$

The diagonal Bertsch-Gunion term contributes only for the first scattering $n=1$ [40, 51]. The off-diagonal terms depend on the current $\mathbf{I}_{n}$, which in turn obeys a recurrence relation itself:

$$
\mathbf{I}_{n}=C_{A}\left(e^{i\left(\omega_{0}-\omega_{n}\right) z_{n}} e^{i \mathbf{q}_{n} \cdot \hat{\mathbf{b}}}-1\right) \mathbf{I}_{n-1}-\delta_{n, 1} C_{A} C_{R} \mathbf{B}_{1} e^{i \omega_{0} z_{1}}
$$

To summarize, the medium-induced bremsstrahlung depends sensitively on the boundary conditions - both at the amplitude and cross sections levels.

Finally, we give one example for the complete solution to the final-state medium-induced bremsstrahlung for a jet produced in a large $Q^{2}$ process. The boundary condition is represented by the amplitude: $J_{0}=-2 i g \frac{\boldsymbol{\epsilon} \cdot \mathbf{k}_{\perp}}{\mathbf{k}_{\perp}^{2}} e^{i \omega_{0} z_{0}} c$, associated with the real hard bremsstrahlung for $x \ll 1$. Rewriting the $z_{n}$ position integrals as integrals over the separation between the scattering centers $\Delta z_{n}=z_{n}-z_{n-1}$ and including the integrals over the momentum distribution of the jet-medium scattering cross section we obtain [40]:

$$
\begin{aligned}
& k^{+} \frac{d N^{g}}{d k^{+} d^{2} \mathbf{k}_{\perp}}=\frac{C_{R} \alpha_{s}}{\pi^{2}} \sum_{n=1}^{\infty}\left[\prod_{i=1}^{n} \int \frac{d \Delta z_{i}}{\lambda_{g}\left(z_{i}\right)}\right]\left[\prod_{j=1}^{n} \int d^{2} \mathbf{q}_{\perp j}\left(\frac{1}{\sigma_{e l}\left(z_{j}\right)} \frac{d \sigma_{e l}\left(z_{j}\right)}{d^{2} \mathbf{q}_{\perp j}}-\delta^{2}\left(\mathbf{q}_{\perp j}\right)\right)\right] \\
& \times\left[-2 \mathbf{C}_{(1, \cdots, n)} \cdot \sum_{m=1}^{n} \mathbf{B}_{(m+1, \cdots, n)(m, \cdots, n)}\left(\cos \left(\sum_{k=2}^{m} \omega_{(k, \cdots, n)} \Delta z_{k}\right)-\cos \left(\sum_{k=1}^{m} \omega_{(k, \cdots, n)} \Delta z_{k}\right)\right)\right] .
\end{aligned}
$$

To use unified notation above, we have to specify $\sum_{2}^{1} \equiv 0$ and $\mathbf{B}_{(n+1, n)} \equiv \mathbf{B}_{n}$. In the case of final-state interactions, $z_{0} \approx 0$ is the point of the initial hard scatter and $z_{L}=L$ is the extent of the medium. The path ordering of the interaction points, $z_{L}>z_{j+1}>z_{j}>z_{0}$, leads to the constraint $\sum_{i=1}^{n} \Delta z_{i} \leq z_{L}$. One implementation of this condition would be $\Delta z_{i} \in\left[0, z_{L}-\sum_{j=1}^{i-1} \Delta z_{j}\right]$ and it is implicit in Eq. (8.21). The complete solution to the problem of medium induced bremsstrahlung in the $x \ll 1$ limit for three different boundary conditions can be found in Ref. [51]. With the proof of the gauge invariance of the reaction operator for collisional and radiative processes, we have also proven the gauge invariance of these results. As we pointed before, when the end result is expresses in terms of physics quantities, such as scattering cross sections, mean free paths and formation times, gauge-invariance can easily be recognized. 


\section{Bremsstrahlung: beyond the soft gluon approximation}

In this section we calculate the corrections to the medium induced bremsstrahlung at finite splitting fraction $x \sim 1$. Effective theory Feynman rules allow us to do so easily. The advantage of the effective theory approach is that we have the appropriate interactions in medium at the Lagrangian level, which allows us to straightforwardly calculate any process of interest. Doing similar calculations, for example calculating radiative energy loss to first order in opacity keeping the full $x$ dependance in the traditional approach would be more difficult, since it will require to do approximations at the level of Feynman diagrams.

\subsection{Incoherent radiation}

We first discuss the simpler case of gluon emission without the Landau-Pomeranchuk-Migdal destructive interference effects. Starting from expressions in Eq. (6.14) -Eq. (6.16) and without making approximation on $x$ we identify three pieces of these matrix elements that are proportional to $\exp \left[i \Omega_{1}\left(z-z_{0}\right)\right]$ (see appendix D.2 for details) as the ones that contribute to the Bertsch-Gunion amplitude - the QCD analog of the Bethe-Heitler radiation in electrodynamics. Squaring the sum of three diagrams and summing over the physical polarizations we obtain:

$$
\frac{1}{d_{R} d_{T}}\left\langle\left|A_{J q \rightarrow J q g}\right|^{2}\right\rangle_{\text {medium } \mathbf{q}_{\perp}}=(1-x) \operatorname{Tr}\left(\frac{\not h}{2} p_{0}^{+} J \bar{J}\right) \times \frac{N}{A_{\perp}} \int d^{2} \mathbf{q}_{\perp} \frac{d \sigma_{e l}^{g \text { medium }}}{d^{2} \mathbf{q}_{\perp}}\left|M_{B G}^{\mathrm{rad}}\right|^{2} .
$$

It easy to see that in Eq. (9.1) $N / A_{\perp}=d z \rho(z)$, where $\rho$ is the density of scattering centers in the medium. Integrating over the path of the quark propagation we find that the differential spectrum of the incoherent Bertsch-Gunion bremsstrahlung can be written as:

$$
x \frac{d N^{g}}{d x d^{2} \boldsymbol{k}_{\perp}}=\int \frac{d z}{\lambda_{g}(z)} \int d^{2} \mathbf{q}_{\perp} \frac{1}{\sigma_{e l}} \frac{d \sigma_{e l}^{g \text { medium }}}{d^{2} \mathbf{q}_{\perp}} \frac{1}{2(2 \pi)^{3}}\left|M_{B G}^{\mathrm{rad}}\right|^{2} .
$$

Here, $\lambda_{g}(z)=1 /\left[\sigma_{e l}^{g \text { medium }} \rho(z)\right]$ and the initial jet direction $\left(p_{0}=p+k\right) \| n$. Very generally, one can express the radiative amplitude squared as follows:

$$
\frac{1}{2(2 \pi)^{3}}\left|M_{B G}^{\mathrm{rad}}\right|^{2}=C_{F} \frac{\alpha_{s}}{\pi^{2}}\left(1-x+\frac{x^{2}}{2}\right) \frac{\mathbf{q}_{\perp}^{2}}{2 \mathbf{A}_{\perp}^{2} \mathbf{B}_{\perp}^{2} \mathbf{C}_{\perp}^{2}}\left[\mathbf{A}_{\perp}^{2}+(1-x)^{2} \mathbf{B}_{\perp}^{2}-\frac{1}{N_{c}^{2}} x^{2} \mathbf{C}_{\perp}^{2}\right]
$$

In Eq. (9.3)

$$
\begin{aligned}
& \mathbf{A}_{\perp}=(1-x) \mathbf{k}_{\perp}-x \mathbf{p}_{\perp}=\left.\mathbf{k}_{\perp}\right|_{\mathbf{p}_{\perp}=-\mathbf{k}_{\perp}}, \\
& \mathbf{B}_{\perp}=(1-x) \mathbf{k}_{\perp}-x\left(\mathbf{p}_{\perp}-\mathbf{q}_{\perp}\right)=\mathbf{k}_{\perp}+x \mathbf{q}_{\perp} \mid \mathbf{p}_{\perp}=-\mathbf{k}_{\perp}, \\
& \mathbf{C}_{\perp}=(1-x)\left(\mathbf{k}_{\perp}-\mathbf{q}_{\perp}\right)-x \mathbf{p}_{\perp}=\mathbf{k}_{\perp}-\left.(1-x) \mathbf{q}_{\perp}\right|_{\mathbf{p}_{\perp}=-\mathbf{k}_{\perp}} .
\end{aligned}
$$

By taking the small $x \ll 1$ limit above and substituting in Eq. (9.3) we obtain the known result:

$$
\frac{1}{2(2 \pi)^{3}}\left|M_{B G}^{\mathrm{rad}}\right|^{2}=C_{F} \frac{\alpha_{s}}{\pi^{2}} \frac{\mathbf{q}_{\perp}^{2}}{\mathbf{k}_{\perp}^{2}\left(\mathbf{k}_{\perp}-\mathbf{q}_{\perp}\right)^{2}} .
$$

The general expression for the incoherent medium-induced gluon bremsstrahlung for a medium of constant density and length $L$ without such approximations is:

$$
\begin{aligned}
x \frac{d N^{g}}{d x d^{2} \boldsymbol{k}_{\perp}}= & C_{F} \frac{\alpha_{s}}{\pi^{2}}\left(1-x+\frac{x^{2}}{2}\right) \frac{L}{\lambda_{g}} \int d^{2} \mathbf{q}_{\perp} \frac{1}{\sigma_{e l}} \frac{d \sigma_{e l}}{d^{2} \mathbf{q}_{\perp}} \frac{\boldsymbol{q}_{\perp}^{2}}{2 \boldsymbol{k}_{\perp}^{2}\left(\boldsymbol{k}_{\perp}+x \boldsymbol{q}_{\perp}\right)^{2}\left(\boldsymbol{k}_{\perp}-(1-x) \boldsymbol{q}_{\perp}\right)^{2}} \\
& \times\left[\left(\boldsymbol{k}_{\perp}^{2}+(1-x)^{2}\left(\boldsymbol{k}_{\perp}+x \boldsymbol{q}_{\perp}\right)^{2}\right)-\frac{1}{N_{c}^{2}} x^{2}\left(\boldsymbol{k}_{\perp}-(1-x) \boldsymbol{q}_{\perp}\right)^{2}\right] .
\end{aligned}
$$



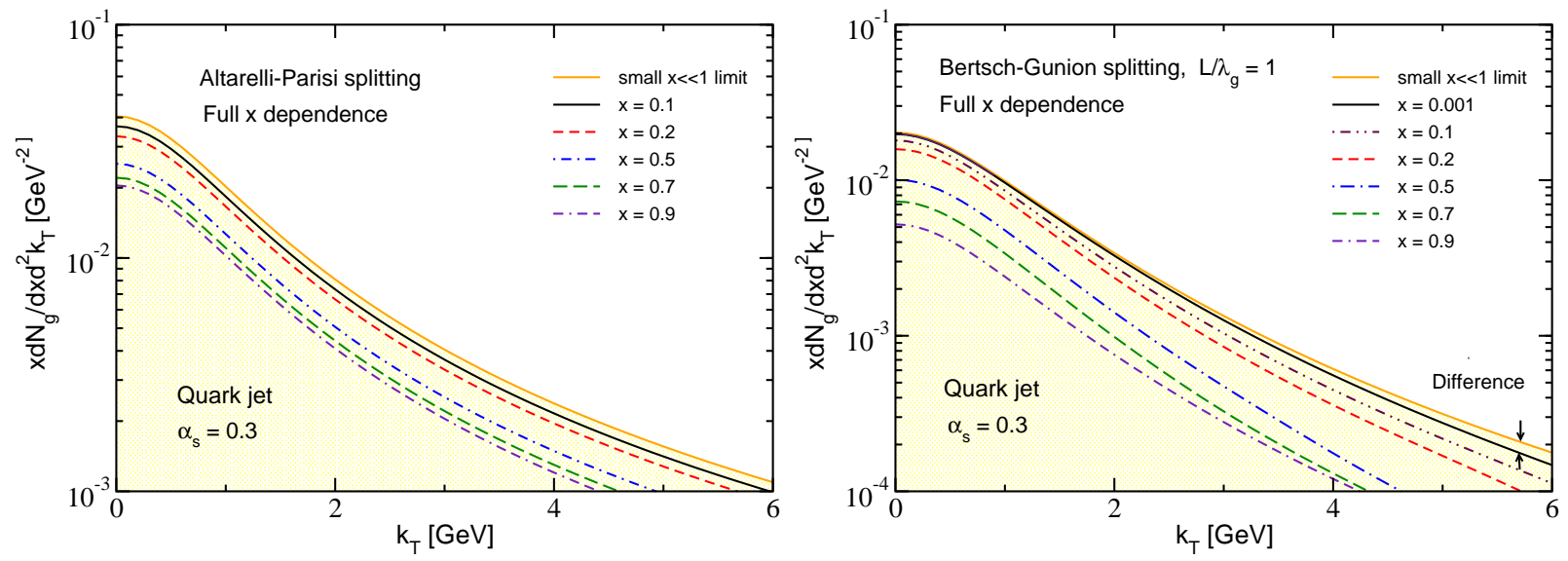

Figure 9: Left panel: the differential gluon number distribution versus $k_{\perp}$ is shown for selected values of $x=k^{+} / p^{+}$ for a quark jet that has undergone large $Q^{2}$ scattering. A finite in-medium mass $m_{\mathrm{eff}}=1 \mathrm{GeV}$ regulates the collinear divergence. Right panel: the same spectrum for the incoherent medium-induced Bertsch-Gunion radiation. We have used $L / \lambda_{g}=1$ and for the $x \rightarrow 0$ also shown an analytic calculation in the massive scattering center limit for comparison to the exact calculation with $m_{\text {med. }}=1 \mathrm{GeV}$.

Finite- $x$ effects are illustrated in figure 9. The left panel shows the Altarelli-Parisi real gluon differential spectrum Eq. (6.11) for selected values of $x=0.1,0.2,0.5,0.7,0.9$ versus $k_{\perp}$. We use $x=0.001$ to simulate the small $x$-independent limit and represent the result by a solid line. The normalization is fixed by the choice of a quark jet and $\alpha_{s}=0.3$. A finite effective mass $m_{\text {eff. }}=1 \mathrm{GeV}$ to simulate medium effects

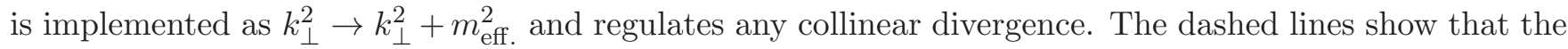
finite- $x$ corrections can be as large as a factor of 2 . The kinematic bound for the transverse momentum of the emitted gluon in large $Q^{2}$ jet production $k_{\perp \text { max }}=\sqrt{x(1-x) Q^{2}}$ is not explicitly shown in the figure.

The right panel of figure 9 shows the medium-induced differential gluon spectrum for the same values of $x$ in the incoherent Bertsch-Gunion limit. We have chosen $L / \lambda_{g}=1$ in Eq. (9.8) to facilitate direct comparison to the Altarelli-Parisi case. In our calculation we used the exact form of the $2 \rightarrow 2$ scattering cross section Eqs. (3.2), (3.3), (3.4), including the finite mass of the medium particles, the medium recoil and the kinematic bounds on $q_{\perp \text { max }}$. Having chosen a quark of energy $E=100 \mathrm{GeV}$, we note that in the Bertsch-Gunion case large- $x$ effects are even more pronounced than in the Altarelli-Parisi case.

It should be noted that if an approximate form for the $q_{\perp}$ dependence of the normalized differential scattering cross section is employed:

$$
\frac{1}{\sigma_{e l}} \frac{d \sigma_{e l}}{d^{2} \mathbf{q}_{\perp}}=\frac{\mu^{2}}{\pi\left(q_{\perp}^{2}+\mu^{2}\right)^{2}}, \quad q_{\perp \max } \rightarrow \infty
$$

the remaining integral in Eq. (9.8) can be performed analytically. This form is motivated by the infinitely massive scattering center approximation. One uses a Feynman change of variables developed for the calculation of loop diagrams. We here quote the final result for the soft gluon approximation $x \ll 1$ :

$$
\begin{aligned}
x \frac{d N^{g}}{d x d^{2} \boldsymbol{k}_{\perp}} \approx & \frac{\alpha_{s} C_{F}}{\pi^{2}}\left(\frac{L}{\lambda_{g}}\right) \frac{\mu^{2}}{\left(\mathbf{k}_{\perp}^{2}\right)^{2}\left(\mathbf{k}_{\perp}^{2}+m_{\text {eff. }}^{2}\right)} \\
& \times \frac{\mu^{2}\left(\lambda_{1}^{2}-\lambda_{2}^{2}\right)+\left[\mu^{2}\left(\lambda_{1}+\lambda_{2}-2\right)+\mathbf{k}_{\perp}^{2}\left(\lambda_{1}-\lambda_{2}\right)^{2}\right] \lambda_{1} \lambda_{2} \ln \left(\frac{\lambda_{1}\left(1-\lambda_{2}\right)}{\lambda_{2}\left(1-\lambda_{1}\right)}\right)}{\lambda_{1} \lambda_{2}\left(\lambda_{1}-\lambda_{2}\right)^{3}},
\end{aligned}
$$


where $\lambda_{1,2}$ are the solutions of the quadratic equation:

$$
\lambda m_{\text {eff. }}^{2}+(1-\lambda) \mu^{2}+\lambda(1-\lambda) \mathbf{k}_{\perp}^{2}=0 .
$$

The analytical formula for the finite $x$ case is also calculable, though slightly more involved. To illustrate the differences between the exact form of the $2 \rightarrow 2$ scattering cross section and the approximate form given by Eq. (9.9) we show the analytic small- $x$ gluon distribution versus $k_{\perp}$ in the right panel of figure 9 . We note that the differences between the exact and approximate calculations are very small and appear only at high $k_{\perp}$ - a part of phase space that does not contribute significantly to the medium-induced energy loss.

\subsection{Final-state radiation and the Landau-Pomeranchuk-Migdal effect}

In this subsection we calculate the combination of single and double Born amplitudes to first order in opacity by keeping the finite- $x$ corrections. This evaluation proceeds analogously to the one for the incoherent Bertsch-Gunion limit. Directly from the Feynman rules of $\mathrm{SCET}_{\mathrm{G}}$, derived in this paper, we find the combined squared amplitude from single and double Born diagrams equals to:

$$
\begin{aligned}
\frac{1}{d_{R} d_{T}}\left\langle\operatorname{Tr}\left[A_{1} A_{1}^{\dagger}+A_{0} A_{2}^{(c) \dagger}+A_{0}^{\dagger} A_{2}^{(c)}\right]\right\rangle_{\text {medium } \mathbf{q}_{\perp}} \\
=\frac{N}{A_{\perp}} \int \frac{d^{2} \boldsymbol{q}_{\perp}}{(2 \pi)^{2}}\left|\tilde{v}\left(\boldsymbol{q}_{\perp}\right)\right|^{2} \operatorname{Tr}\left(\frac{\not h}{2} \bar{n} \cdot p J \bar{J} \frac{g^{2}}{d_{R} d_{T}}\left[\rho^{\mathrm{SB}}+\rho^{\mathrm{DB}}\right]\right),
\end{aligned}
$$

where both $\rho^{\mathrm{SB}}$ and $\rho^{\mathrm{DB}}$ have the following form:

$$
\rho=\sum_{i=1}^{2} c_{i}\left(F_{i} \mathbb{I}+G_{i} \Sigma^{3}\right) .
$$

In the equation above $F_{i}, G_{i}$ are form-factors, which we give below, and $c_{i}$ are the color factors:

$$
c_{1}=C_{2}(R)^{2} C(r) d_{R}, \quad c_{2}=C_{2}(R) C(r)\left(C_{2}(R)-\frac{1}{2} C_{A}\right) d_{R}
$$

We recall that in our example $C_{2}(R)=C_{F}, d_{R}=N_{c}$ and $C(r)=\frac{1}{2}$. The two operators that appear are the identity operator in the Dirac indices $\mathbb{I}$ and $\Sigma^{3}$, which is the third component of the spin operator for the fermion:

$$
\Sigma^{3}=\left[\begin{array}{cc}
\sigma_{3} & 0 \\
0 & \sigma_{3}
\end{array}\right]
$$

The first term in Eq. (9.13) allows for the factorization of the medium-induced radiative corrections and the hard scattering cross section even in the large- $x$ limit. The second term in principle does not vanish. If we, however, recall the decomposition of $J(p) \bar{J}(p)$ in the Dirac algebra basis, see Eq. (5.13), it is easy to verify that there must be a non-zero pseudo-vector contribution for this term not to vanish identically. Therefore, one might expect further corrections for processes with electroweak boson exchanges. For QCD, medium-induced radiative corrections always factorize. A simple exercise for the interested reader is to show this on the example of inclusive tree level jet production. 
The form-factors for the single Born diagrams in Eq. (9.13) are given by the following expressions:

$$
\begin{aligned}
& F_{1}^{\mathrm{SB}}=\left(1-x+\frac{x^{2}}{2}\right)\left(\left|\boldsymbol{\beta}_{1}\right|^{2}+\left|\boldsymbol{\beta}_{2}\right|^{2}\right), \quad G_{1}^{\mathrm{SB}}=2\left(x-\frac{x^{2}}{2}\right) \operatorname{Im}\left[\left(\boldsymbol{\beta}_{1}^{x}\right)^{*} \boldsymbol{\beta}_{1}^{y}+\left(\boldsymbol{\beta}_{2}^{x}\right)^{*} \boldsymbol{\beta}_{2}^{y}\right], \\
& F_{2}^{\mathrm{SB}}=\left(1-x+\frac{x^{2}}{2}\right)\left(\boldsymbol{\beta}_{1} \cdot \boldsymbol{\beta}_{2}^{*}+\boldsymbol{\beta}_{1}^{*} \cdot \boldsymbol{\beta}_{2}\right),
\end{aligned}
$$

On the other hand, the double Born diagrams contributions can be written as follows:

$$
\begin{array}{ll}
F_{1}^{\mathrm{DB}}=\left(1-x+\frac{x^{2}}{2}\right)\left(\boldsymbol{\alpha}_{0} \cdot \boldsymbol{\gamma}_{1}^{*}+\boldsymbol{\alpha}_{0}^{*} \cdot \boldsymbol{\gamma}_{1}\right), & G_{1}^{\mathrm{DB}}=2\left(x-\frac{x^{2}}{2}\right) \operatorname{Im}\left[\left(\boldsymbol{\gamma}_{1}^{x}\right)^{*} \boldsymbol{\alpha}_{0}^{y}+\left(\boldsymbol{\alpha}_{0}^{x}\right)^{*} \boldsymbol{\gamma}_{1}^{y}\right], \\
F_{2}^{\mathrm{DB}}=\left(1-x+\frac{x^{2}}{2}\right)\left(\boldsymbol{\alpha}_{0} \cdot \boldsymbol{\gamma}_{2}^{*}+\boldsymbol{\alpha}_{0}^{*} \cdot \boldsymbol{\gamma}_{2}\right), & G_{2}^{\mathrm{DB}}=2\left(x-\frac{x^{2}}{2}\right) \operatorname{Im}\left[\left(\boldsymbol{\gamma}_{2}^{x}\right)^{*} \boldsymbol{\alpha}_{0}^{y}+\left(\boldsymbol{\alpha}_{0}^{x}\right)^{*} \boldsymbol{\gamma}_{2}^{y}\right] .
\end{array}
$$

In the expressions above the vector $\boldsymbol{\alpha}_{0}$ appears from the vacuum diagram, the vectors $\boldsymbol{\beta}_{1,2}$ appear from the single Born diagrams and $\gamma_{1,2}$ appear from the double Born diagrams calculation. They all are given in the equations below:

$$
\begin{aligned}
\boldsymbol{\alpha}_{0} & =\frac{2 \boldsymbol{A}_{\perp}}{\boldsymbol{A}_{\perp}^{2}} \\
\boldsymbol{\beta}_{1} & =\left(\frac{2 \boldsymbol{A}_{\perp}}{\boldsymbol{A}_{\perp}^{2}}-\frac{2 \boldsymbol{C}_{\perp}}{\boldsymbol{C}_{\perp}^{2}}\right) \mathrm{e}^{i \Omega_{1} \delta z}+\frac{2 \boldsymbol{C}_{\perp}}{\boldsymbol{C}_{\perp}^{2}} \mathrm{e}^{i \Omega_{3} \delta z} \\
\boldsymbol{\beta}_{2} & =-\left(\frac{2 \boldsymbol{B}_{\perp}}{\boldsymbol{B}_{\perp}^{2}}-\frac{2 \boldsymbol{C}_{\perp}}{\boldsymbol{C}_{\perp}^{2}}\right) \mathrm{e}^{i \Omega_{1} \delta z}+\frac{2 \boldsymbol{B}_{\perp}}{\boldsymbol{B}_{\perp}^{2}} \mathrm{e}^{i \Omega_{2} \delta z}-\frac{2 \boldsymbol{C}_{\perp}}{\boldsymbol{C}_{\perp}^{2}} \mathrm{e}^{i \Omega_{3} \delta z} \\
\gamma_{1} & =-\frac{3 \boldsymbol{A}_{\perp}}{\boldsymbol{A}_{\perp}^{2}}+\left(\frac{2 \boldsymbol{A}_{\perp}}{\boldsymbol{A}_{\perp}^{2}}-\frac{2 \boldsymbol{D}_{\perp}}{\boldsymbol{D}_{\perp}^{2}}\right) \mathrm{e}^{i \Omega_{4} \delta z}+\frac{2 \boldsymbol{D}_{\perp}}{\boldsymbol{D}_{\perp}^{2}} \mathrm{e}^{i \Omega_{5} \delta z} \\
\gamma_{2} & =\frac{2 \boldsymbol{A}_{\perp}}{\boldsymbol{A}_{\perp}^{2}}-\left(\frac{2 \boldsymbol{A}_{\perp}}{\boldsymbol{A}_{\perp}^{2}}-\frac{2 \boldsymbol{D}_{\perp}}{\boldsymbol{D}_{\perp}^{2}}\right) \mathrm{e}^{i \Omega_{4} \delta z}-\frac{2 \boldsymbol{D}_{\perp}}{\boldsymbol{D}_{\perp}^{2}} \mathrm{e}^{i \Omega_{5} \delta z}
\end{aligned}
$$

where the vectors $\boldsymbol{A}_{\perp}, \boldsymbol{B}_{\perp}, \boldsymbol{C}_{\perp}$ are same as in the previous subsection, the phase factors $\Omega_{1,2,3}$ are same as in appendix D.2. The remaining propagators and inverse formation times $\boldsymbol{D}_{\perp}, \Omega_{4}, \Omega_{5}$ appear in the double Born diagrams and are equal to:

$$
\begin{aligned}
& \boldsymbol{D}_{\perp}=\boldsymbol{A}_{\perp}-\boldsymbol{q}_{\perp}=\boldsymbol{k}_{\perp}-\left.\boldsymbol{q}_{\perp}\right|_{\mathbf{p}_{\perp}=-\mathbf{k}_{\perp}} \\
& \Omega_{4}=\frac{\boldsymbol{A}_{\perp}^{2}}{p_{0}^{+} x(1-x)}, \quad \Omega_{5}=\frac{\boldsymbol{A}_{\perp}^{2}-\boldsymbol{D}_{\perp}^{2}}{p_{0}^{+} x(1-x)} .
\end{aligned}
$$

First, we should check that our result agrees with previous calculation in the soft gluon approximation $[51,40]$. For this we need to expand all quantities to lowest order in $x \ll 1$ :

$$
\begin{aligned}
& \boldsymbol{A}_{\perp} \approx \boldsymbol{k}_{\perp}, \quad \boldsymbol{B}_{\perp} \approx \boldsymbol{k}_{\perp}, \quad \boldsymbol{C}_{\perp} \approx \boldsymbol{k}_{\perp}-\boldsymbol{q}_{\perp}, \quad \boldsymbol{D}_{\perp} \approx \boldsymbol{k}_{\perp}-\boldsymbol{q}_{\perp}, \\
& \Omega_{1} \approx \omega_{0}, \quad \Omega_{2} \approx 0, \quad \Omega_{3} \approx \omega_{0}-\omega_{1}, \quad \Omega_{4} \approx \omega_{0}, \quad \Omega_{5} \approx \omega_{0}-\omega_{1}, \\
& G_{i}^{\mathrm{SB}} \approx 0, \quad G_{i}^{\mathrm{DB}} \approx 0 .
\end{aligned}
$$

Note that in this limit factorization is exact even if $J(p) \bar{J}(p)$ contains a pseudo-vector component. The final answer depends on the form-factors $F_{i}$, which we calculate below in the soft gluon limit. It is convenient 
to rewrite the $\boldsymbol{\alpha}, \boldsymbol{\beta}_{1,2}, \boldsymbol{\gamma}_{1,2}$ in terms of standard definitions in the literature ${ }^{16}[51,40]$ :

$$
\boldsymbol{H}_{1}=\frac{\boldsymbol{k}_{\perp}}{\boldsymbol{k}_{\perp}^{2}}, \quad \boldsymbol{C}_{1}=\frac{\boldsymbol{k}_{\perp}-\boldsymbol{q}_{\perp}}{\left(\boldsymbol{k}_{\perp}-\boldsymbol{q}_{\perp}\right)^{2}}, \quad \boldsymbol{B}_{1}=\boldsymbol{H}_{1}-\boldsymbol{C}_{1} .
$$

The corresponding $x \rightarrow 0$ limit is particularly simple:

$$
\begin{aligned}
& \boldsymbol{\alpha}_{0}=2 \boldsymbol{H}_{1}, \\
& \boldsymbol{\beta}_{1}=2 \boldsymbol{B}_{1} \mathrm{e}^{i \omega_{0} \delta z}+2 \boldsymbol{C}_{1} \mathrm{e}^{i\left(\omega_{0}-\omega_{1}\right) \delta z}, \quad \boldsymbol{\beta}_{2}=2 \boldsymbol{H}_{1}-2 \boldsymbol{B}_{1} \mathrm{e}^{i \omega_{0} \delta z}-2 \boldsymbol{C}_{1} \mathrm{e}^{i\left(\omega_{0}-\omega_{1}\right) \delta z}, \\
& \boldsymbol{\gamma}_{1}=-3 \boldsymbol{H}_{1}+2 \boldsymbol{B}_{1} \mathrm{e}^{i \omega_{0} \delta z}+2 \boldsymbol{C}_{1} \mathrm{e}^{i\left(\omega_{0}-\omega_{1}\right) \delta z}, \quad \boldsymbol{\gamma}_{2}=2 \boldsymbol{H}_{1}-2 \boldsymbol{B}_{1} \mathrm{e}^{i \omega_{0} \delta z}-2 \boldsymbol{C}_{1} \mathrm{e}^{i\left(\omega_{0}-\omega_{1}\right) \delta z} .
\end{aligned}
$$

Useful relations between these vectors, that help in deriving the expression for the form-factors $F_{i}$ are the following ones:

$$
\boldsymbol{\beta}_{1}+\boldsymbol{\beta}_{2}=2 \boldsymbol{H}_{1}, \quad \gamma_{1}+\gamma_{2}=-\boldsymbol{H}_{1} .
$$

From the definitions in Eq. (9.15)-Eq. (9.18) we get:

$$
\begin{aligned}
F_{1}^{\mathrm{SB}} & =\left|\boldsymbol{\beta}_{1}\right|^{2}+\left|2 \boldsymbol{H}_{1}-\boldsymbol{\beta}_{1}\right|^{2}=2\left|\boldsymbol{\beta}_{1}\right|^{2}+4 \boldsymbol{H}_{1}^{2}-4 \operatorname{Re} \boldsymbol{H}_{1} \cdot \boldsymbol{\beta}_{1} \\
& =8 \boldsymbol{B}_{1}^{2}+8 \boldsymbol{C}_{1}^{2}+4 \boldsymbol{H}_{1}^{2}+16 \boldsymbol{B}_{1} \cdot \boldsymbol{C}_{1} \cos \left(\omega_{1} \delta z\right)-8 \boldsymbol{H}_{1} \cdot \boldsymbol{B}_{1} \cos \left(\omega_{0} \delta z\right)-8 \boldsymbol{H}_{1} \cdot \boldsymbol{C}_{1} \cos \left(\left(\omega_{0}-\omega_{1}\right) \delta z\right), \\
F_{2}^{\mathrm{SB}} & =\boldsymbol{\beta}_{1}\left(2 \boldsymbol{H}_{1}-\boldsymbol{\beta}_{1}\right)^{*}+\left(2 \boldsymbol{H}_{1}-\boldsymbol{\beta}_{2}\right)^{*} \boldsymbol{\beta}_{2}=-F_{1}^{\mathrm{SB}}+4 \boldsymbol{H}_{1}^{2}, \\
F_{1}^{\mathrm{DB}} & =2 \boldsymbol{H}_{1} \cdot 2 \operatorname{Re} \boldsymbol{\gamma}_{1}=-12 \boldsymbol{H}_{1}^{2}+8 \boldsymbol{H}_{1} \cdot \boldsymbol{B}_{1} \cos \left(\omega_{0} \delta z\right)+8 \boldsymbol{H}_{1} \cdot \boldsymbol{C}_{1} \cos \left(\left(\omega_{0}-\omega_{1}\right) \delta z\right), \\
F_{2}^{\mathrm{DB}} & =2 \boldsymbol{H}_{1} \cdot 2 \operatorname{Re} \boldsymbol{\gamma}_{2}=-F_{1}^{\mathrm{DB}}-4 \boldsymbol{H}_{1}^{2} .
\end{aligned}
$$

Finally, using these equations we combine the single and double Born form-factors into the sum:

$$
\begin{aligned}
& F_{1}^{\mathrm{SB}}+F_{1}^{\mathrm{DB}}=8 \boldsymbol{B}_{1}^{2}+8 \boldsymbol{C}_{1}^{2}-8 \boldsymbol{H}_{1}^{2}+16 \boldsymbol{B}_{1} \cdot \boldsymbol{C}_{1} \cos \left(\omega_{1} \delta z\right)=-16 \boldsymbol{B}_{1} \cdot \boldsymbol{C}_{1}\left(1-\cos \left(\omega_{1} \delta z\right)\right), \\
& F_{2}^{\mathrm{SB}}+F_{2}^{\mathrm{DB}}=-F_{1}^{\mathrm{SB}}-F_{1}^{\mathrm{DB}} .
\end{aligned}
$$

Thus, in the soft gluon approximation we get:

$$
\left(\rho^{\mathrm{SB}}+\rho^{\mathrm{DB}}\right)_{x \ll 1} \approx\left(c_{1}-c_{2}\right)\left(-16 \boldsymbol{B}_{1} \cdot \boldsymbol{C}_{1}\right)\left(1-\cos \left(\omega_{1} \delta z\right)\right) .
$$

Taking into account the phase space factors, the color factors and the final-state coherent medium-induced emission contribution above, we find:

$$
x \frac{d N^{g}}{d x d^{2} \boldsymbol{k}_{\perp} \mid x \ll 1}=C_{F} \frac{\alpha_{s}}{\pi^{2}} \int \frac{d \Delta z}{\lambda_{g}(z)} \int d^{2} \mathbf{q}_{\perp} \frac{1}{\sigma_{e l}} \frac{d \sigma_{e l}^{g \text { medium }}}{d^{2} \mathbf{q}_{\perp}}\left(-2 \boldsymbol{B}_{1} \cdot \boldsymbol{C}_{1}\right)\left(1-\cos \left(\omega_{1} \Delta z\right)\right) .
$$

in agreement with Eq. (70) of [51].

Beyond the soft gluon approximation, the full result for the coherent medium-induced bremsstrahlung reads:

$$
\begin{aligned}
x \frac{d N^{g}}{d x d^{2} \boldsymbol{k}_{\perp}}= & C_{F} \frac{\alpha_{s}}{\pi^{2}}\left(1-x+\frac{x^{2}}{2}\right) \int \frac{d \Delta z}{\lambda_{g}(z)} \int d^{2} \mathbf{q}_{\perp} \frac{1}{\sigma_{e l}} \frac{d \sigma_{e l}^{g}}{d^{2} \mathbf{q}_{\perp}}\left[-\left(\frac{\boldsymbol{A}_{\perp}}{\boldsymbol{A}_{\perp}^{2}}\right)^{2}+2\left(\frac{\boldsymbol{C}_{\perp}}{\boldsymbol{C}_{\perp}^{2}}\right)^{2}-\frac{\boldsymbol{A}_{\perp}}{\boldsymbol{A}_{\perp}^{2}} \cdot \frac{\boldsymbol{C}_{\perp}}{\boldsymbol{C}_{\perp}^{2}}\right. \\
& -\frac{\boldsymbol{B}_{\perp}}{\boldsymbol{B}_{\perp}^{2}} \cdot \frac{\boldsymbol{C}_{\perp}}{\boldsymbol{C}_{\perp}^{2}}\left(1-\cos \left[\left(\Omega_{1}-\Omega_{2}\right) \Delta z\right]+\cos \left[\left(\Omega_{2}-\Omega_{3}\right) \Delta z\right]\right) \\
& +\frac{\boldsymbol{C}_{\perp}}{\boldsymbol{C}_{\perp}^{2}} \cdot\left(\frac{\boldsymbol{A}_{\perp}}{\boldsymbol{A}_{\perp}^{2}}+\frac{\boldsymbol{B}_{\perp}}{\boldsymbol{B}_{\perp}^{2}}-2 \frac{\boldsymbol{C}_{\perp}}{\boldsymbol{C}_{\perp}^{2}}\right) \cos \left[\left(\Omega_{1}-\Omega_{3}\right) \Delta z\right]+\frac{\boldsymbol{A}_{\perp}}{\boldsymbol{A}_{\perp}^{2}} \cdot\left(\frac{\boldsymbol{A}_{\perp}}{\boldsymbol{A}_{\perp}^{2}}-\frac{\boldsymbol{D}_{\perp}}{\boldsymbol{D}_{\perp}^{2}}\right) \cos \left[\Omega_{4} \Delta z\right] \\
& \left.+\frac{\boldsymbol{A}_{\perp}}{\boldsymbol{A}_{\perp}^{2}} \cdot \frac{\boldsymbol{D}_{\perp}}{\boldsymbol{D}_{\perp}^{2}} \cos \left[\Omega_{5} \Delta z\right]+\left(\frac{N_{c}^{2}-1}{N_{c}^{2}}\left(\frac{\boldsymbol{B}_{\perp}}{\boldsymbol{B}_{\perp}^{2}}\right)^{2}+\frac{1}{N_{c}^{2}} \frac{\boldsymbol{A}_{\perp}}{\boldsymbol{A}_{\perp}^{2}} \cdot \frac{\boldsymbol{B}_{\perp}}{\boldsymbol{B}_{\perp}^{2}}\right)\left(1-\cos \left[\left(\Omega_{1}-\Omega_{2}\right) \Delta z\right]\right)\right] .(9.4)
\end{aligned}
$$

\footnotetext{
${ }^{16}$ Note that $\boldsymbol{B}_{1}$ is distinct from $\boldsymbol{B}_{\perp}$ and $\boldsymbol{C}_{1}$ is distinct from $\boldsymbol{C}_{\perp}$.
} 
We leave the discussion of this new result and phenomenological applications to new RHIC and LHC experimental data $[29,30,31,32,33,34]$ for future work. We note however that in Ref. [57] an evaluation of the medium-induced energy loss beyond the helicity amplitude approximation found $\sim 18 \%$ reduction in the mean medium-induced energy loss. Our plan for the future is to investigate the large- $x$ radiative correction reduction effects at the most differential level.

\section{Conclusions}

In summary, we constructed an effective theory $\operatorname{SCET}_{\mathrm{G}}$ for energetic quark and gluon $p \sim\left[1, \lambda^{2}, \boldsymbol{\lambda}\right]$ propagation and interaction in dense QCD matter. This theory is well-suited to calculations both in the quark-gluon plasma $[25,26,27,28,56]$ and in cold nuclear matter $[38,55,56,58]$.

To construct this theory, we examined the relevant $t$-channel parton scattering cross sections and demonstrated that they are dominated by forward scattering. The corresponding momentum exchanges $q \sim\left[\lambda^{2}, \lambda^{2}, \boldsymbol{\lambda}\right]$ are approximately transverse to the direction of jet propagation and mediated by Glauber gluons. We also demonstrated that a fully dynamical treatment of the scattering centers in QCD matter leads to a small reduction of the scattering cross section and smaller medium-induced effects in contrast to early speculations [43]. The $\mathrm{SCET}_{\mathrm{G}}$ Lagrangian was shown to be invariant under soft and collinear gauge transformations. We derived the Feynman rules for this new Lagrangian in the covariant and light-cone gauges. Also we provided a third choice, which we call the hybrid gauge, when the Glauber gluons are quantized in the covariant gauge, while the collinear fields are gauge-fixed with the light-cone gauge. This choice provides us with the simplest form of Feynman rules and is useful from practical point of view.

The new effective theory was used to evaluate the broadening and medium-induced radiation for energetic quarks traversing a region of hot/dense QCD matter. SCET $\mathrm{G}$ was shown to recover exactly the known results for the transverse momentum diffusion of particles in the strongly-interacting medium [37, 38]. It allows to study the Molliere multiple parton scattering beyond the limitations of the Gaussian approximation - namely, the inability to describe the Rutherford power-law tails of transverse momentum distributions. For the case of inelastic quark interactions we derived the fully differential medium-induces gluon bremsstrahlung spectrum for both the incoherent and Landau-Pomeranchuck-Migdal suppressed cases. In the soft gluon approximation we obtained the kernel for the reaction operator approach to medium-induced energy loss $[40,51]$. We also evaluated the large $x=k^{+} / E^{+}$corrections to the mediuminduced bremsstrahlung spectrum. Gauge invariance of the jet broadening and radiative energy loss results was demonstrated explicitly for the first time by performing the calculations in the covariant $R_{\xi}$, lightcone and hybrid gauges. On the example of an energetic quark, we found that in QCD the processdependent medium-induced radiative corrections factorize from the hard jet production cross section. This allows us to write down perturbative jet and leading particle observables in heavy ion collisions as a convolution of the corresponding observables in the more elementary nucleon-nucleon reactions and the medium-induced collisional and radiative corrections specific to the process under investigation. Our results put jet quenching phenomenology in heavy-ion collisions on more solid theoretical grounds.

In the near future it will be a high priority for us to implement the improved theory of parton propagation and energy loss in matter in perturbative QCD predictions for analysis of the energetic particle and jet quenching data from the heavy-ion experiments at RHIC and at the LHC. With the calculations at hand, we expect to be able to reliably combine the process-dependent medium-induced radiative corrections with next-to-leading order perturbative effects [59]. We plan to extend these calculations beyond applications 
to jet propagation in the quark-gluon plasma $[25,26,28]$ and also improve the accuracy in the evaluation of cold nuclear matter effects [58].

The derived Lagrangian of $\mathrm{SCET}_{\mathrm{G}}$ can be used to revisit the factorization of the Drell-Yan process in the effective theory and include the spectator interactions into the analysis. The importance of Glauber (or Coulomb) gluons for the Drell-Yan factorization has been addressed, and their cancellation in the inclusive cross section has been proved in traditional QCD approach to factorization [47, 48, 49], while similar understanding in effective theory method is still missing [44, 46].

\section{Acknowledgments}

We thank Christian Bauer and Gerry Hale for many illuminating discussions. We thank Christian Bauer for useful comments on the manuscript. This research is supported by the US Department of Energy, Office of Science, under Contract No. DE-AC52-06NA25396 and in part by the LDRD program at LANL.

\section{A. Light-cone notation}

The momentum of any particle can be conveniently represented in light-cone coordinates. A light-cone vector $\beta$ is defined by the condition $\beta^{2}=0$. The two vectors that specify the positive and negative lightcone directions are $n^{\mu}=(1,0,0,1)$ and $\bar{n}^{\mu}=(1,0,0,-1)$, respectively. An arbitrary four-vector $p$ then can be expanded in the light-cone vectors basis:

$$
p^{\mu}=p^{+} \frac{n^{\mu}}{2}+p^{-} \frac{\bar{n}^{\mu}}{2}+p_{\perp}^{\mu},
$$

where the,+- components are defined as follows: $p^{+} \equiv \bar{n} \cdot p, p^{-} \equiv n \cdot p$. The four-vector $p^{\mu}$ thus can be written in the light-cone basis as $\left[p^{+}, p^{-}, \boldsymbol{p}_{\perp}\right]$, where we will use the square brackets to emphasize that the light-cone notation is being used. For example, the light-cone vectors have the following coordinates: $n=[2,0, \mathbf{0}], \bar{n}=[0,2, \mathbf{0}]$, corresponding to positive and negative light-cone directions. Thus, the plus component is in the $n$ direction and the minus component is in the $\bar{n}$ direction. The degrees of freedom of SCET are collinear quarks and gluons, with the following momentum scaling $p_{c}=\left[1, \lambda^{2}, \boldsymbol{\lambda}\right]$ and soft gluons with momentum $p_{s}=\left[\lambda^{2}, \lambda^{2}, \lambda^{2}\right]$. The Glauber modes that we consider in this paper have momentum scaling $p_{g}=\left[\lambda^{2}, \lambda^{2}, \boldsymbol{\lambda}\right]$.

\section{B. Kinematics in the laboratory frame}

In section 3 we estimated the recoil effect in $2 \rightarrow 2$ scattering of a projectile particle with mass $m_{1}$ on an originally at rest target particle with the mass $m_{2}$. An important feature of the laboratory frame is that the final energy $E_{3}$ of the scattered particle is a non-trivial function of the laboratory angle $\theta$. This function can be found from energy and momentum conservation laws. The solution is given by:

$$
E_{3}(\theta)=m_{2} \frac{\rho_{1} \gamma^{2} \pm\left(\gamma^{2}-1\right) r \sin \theta \cos \theta}{\gamma^{2}-\left(\gamma^{2}-1\right) \cos ^{2} \theta} .
$$

In Eq. (B.1) we defined:

$$
\begin{aligned}
& \rho_{1}=\frac{\beta}{\beta_{1}}, \\
& r=\sqrt{\gamma^{2}\left(1-\rho_{1}^{2}\right)+\cot ^{2} \theta},
\end{aligned}
$$


where $\beta_{1}$ is the velocity of the projectile particle in the center-of-mass (CM) frame and $\beta$ is the velocity of this center-of-mass. Note that the kinematics is very different depending on the masses. If $m_{1}>m_{2}$, then both solutions in Eq. (B.1) are physical, while for $m_{1}<m_{2}$ only the "+" solution is physical, while the "-"-solution is not. In our case we can safely assume that $m_{1}<m_{2}$.

The reason for the difference discussed above is that when the projectile particle is heavier then the target one, then in the center of mass frame the projectile particle's velocity is smaller than the centerof-mass velocity in the laboratory frame, i.e $\beta_{1}<\beta$. This means that no matter in which direction the projectile particle goes in the CM frame, it will always move in the positive hemisphere of the laboratory frame with respect to the initial momentum of the projectile particle. Thus, each direction of motion of the scattered projectile particle in the laboratory frame corresponds to two distinct directions in the center of mass frame. That is the origin of two solutions from Eq. (B.1). Alternatively, for $m_{1}<m_{2}$, we have $\beta_{1}>\beta$ and each direction of the projectile particle in laboratory frame comes from a distinct direction of this particle in the center of mass frame, leading to the corresponding unique energy in the laboratory frame.

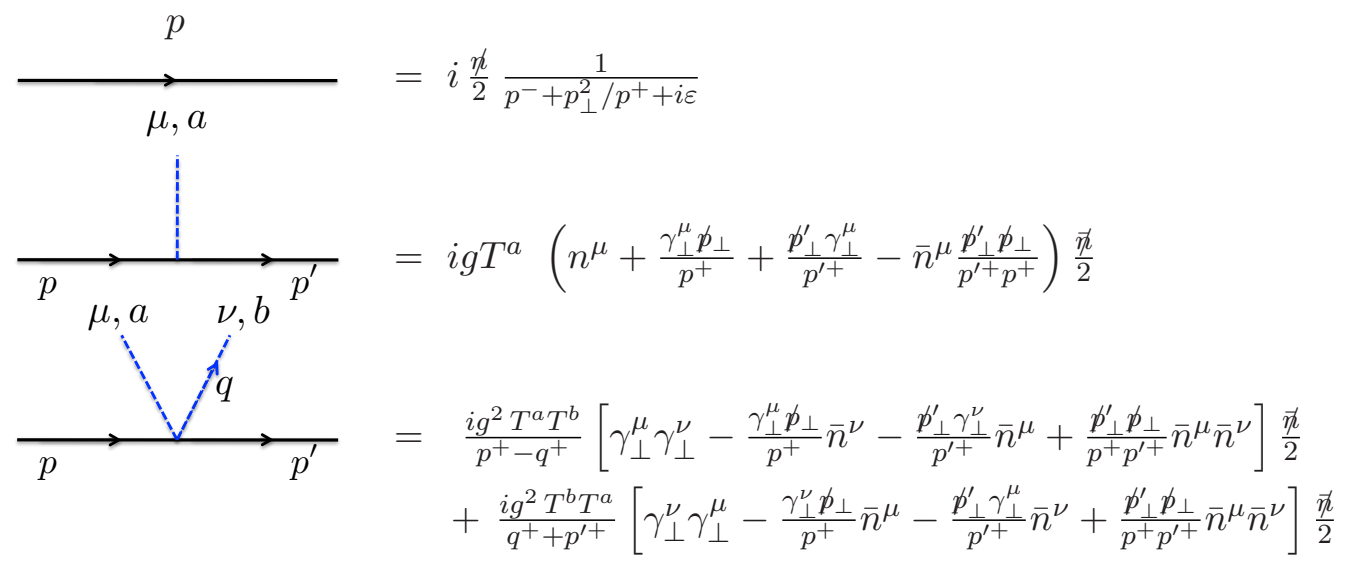

Figure 10: Feynman rules of SCET for the interactions between collinear quarks and gluons.

\section{Feynman rules of $\mathrm{SCET}_{\mathrm{G}}$}

In this appendix we review the Feynman rules of SCET and also present all the relevant Feynman rules of $\mathrm{SCET}_{\mathrm{G}}$ for different gauge choices. We consider the initially static source case.

First, we start with the SCET rules. Using the Lagrangian of SCET [9] given in Eq. (2.1) one finds the Feynman rules of interaction between the collinear quarks and gluons given in figure 10. These rules are given in the covariant gauge. There are additional vertices in this gauge when one has more than 2 collinear gluons and two collinear quarks at the same point, which we omit here. The same Feynman rules as in figure 10 are valid in the positive light-cone gauge $A^{+}$, with the simplification that all the $\bar{n}^{\mu}, \bar{n}^{\nu}$ terms vanish. Also, all the mentioned omitted vertices vanish in the light-cone gauge.

Our approach to deriving the effective Lagrangian of $\mathrm{SCET}_{\mathrm{G}}$ was to treat the effect from Glauber gluons on the target jet as one of an external background field, which the source creates. Having determined the scaling of the vector potential, we then read off the Glauber term from the SCET Lagrangian as it is given in Eq. (2.1) with a trivial addition of an external vector potential to the covariant derivative. We 


$$
\begin{aligned}
& \underset{R_{\xi}}{\stackrel{p}{\longrightarrow} p^{\prime}}=i v\left(q_{1 \perp}\right)\left(b_{1}\right)_{R}\left(b_{1}\right)_{T_{i}} \frac{\vec{n}}{2}
\end{aligned}
$$

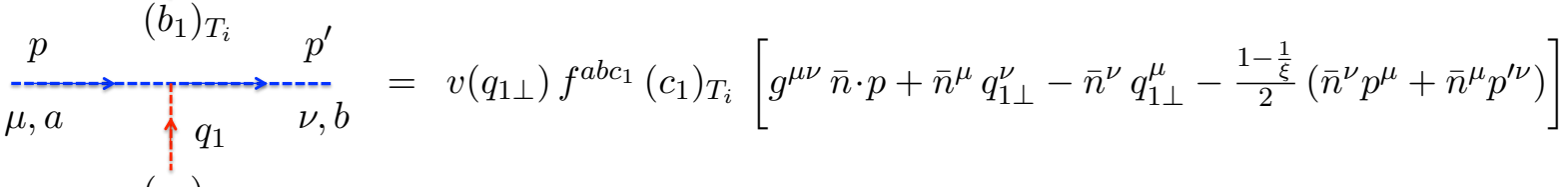

$$
\begin{aligned}
& \left(c_{1}\right)_{T_{i}}
\end{aligned}
$$

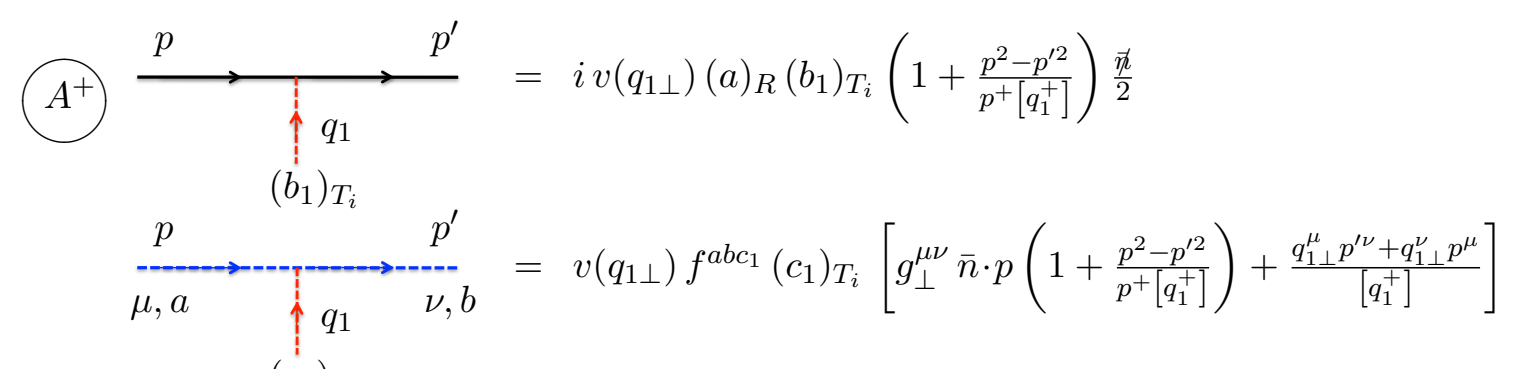$$
\stackrel{p \longrightarrow p^{\prime}}{\longrightarrow}=i v\left(q_{1 \perp}\right) v\left(q_{2 \perp}\right)\left(b_{1} b_{2}\right)_{R}\left(b_{1}\right)_{T_{i}}\left(b_{2}\right)_{T_{j}} \frac{2 q_{1 \perp} \cdot q_{2} \perp}{p^{+}\left[q_{1}^{+}\right]\left[q_{2}^{+}\right]^{\frac{n n}{2}}}
$$

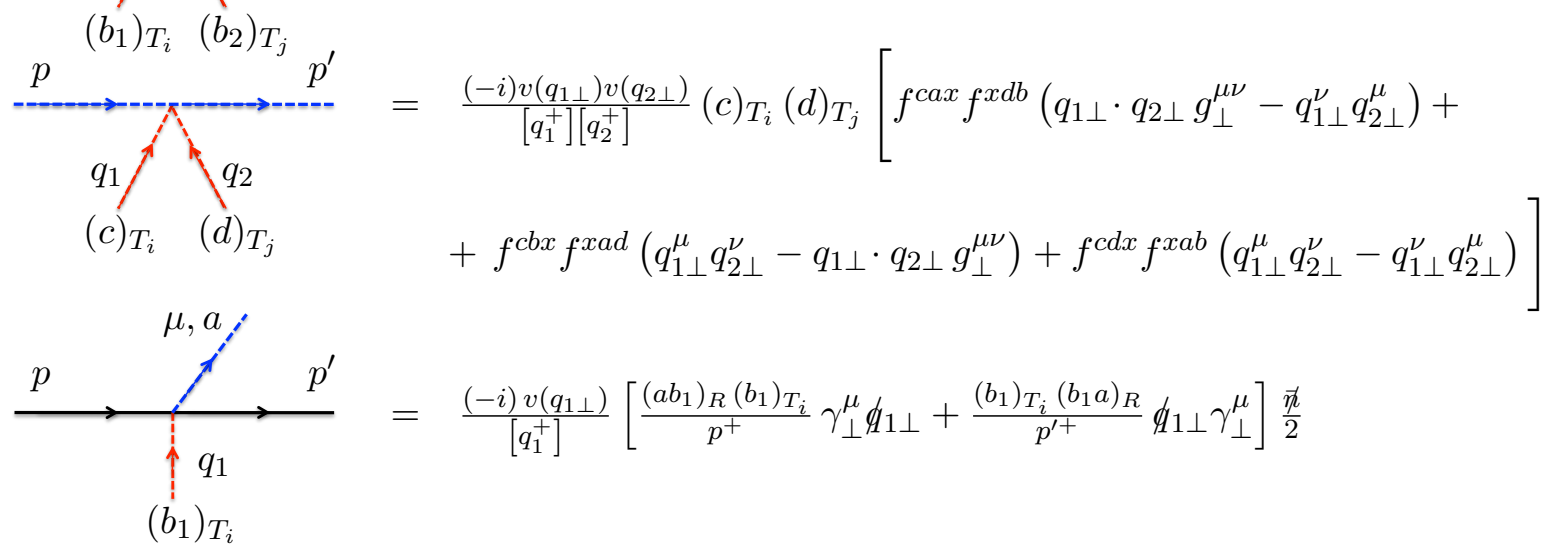

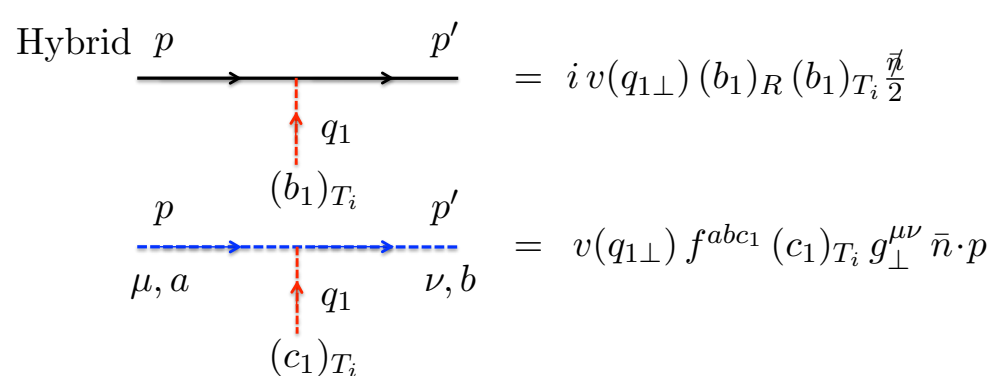

Figure 11: Feynman rules of $\mathrm{SCET}_{\mathrm{G}}$ involving Glauber gluons. The first two vertices are given in the covariant gauge. Vertices from three through seven are for the light-cone gauge $A^{+}=0$. Last two vertices are for the Hybrid gauge. Note that the last three vertices in the light-cone gauge are power-suppressed in the covariant and Hybrid gauges. 
present in figure 11 the resulting Feynman rules. Note that we include the propagator of the Glauber gluon into the Feynman rule as well as the color and overall Dirac structure from the static fermionic source that we consider.

In the main part of the paper we consider three gauge choices. First, we used the covariant gauge in the sections 5, 6. Second, we used the light-cone gauge and third was the hybrid gauge in section 7 . In the first case we used covariant gauge for both pure SCET and the Glauber interactions given in figure 11. In the second case we did the same for the light-cone gauge. Finally, for the hybrid gauge, we used the light-cone gauge for SCET gauge fixing, while using the covariant gauge for Glauber gluons. In all three cases we found the same results for physical quantities, as expected.

\section{Longitudinal integrals}

In this appendix we define and calculate all longitudinal momentum integrals that are required for the evaluation of jet broadening and radiative energy loss. Our notation is $I_{m}^{(n)}$, where $n$ indicates number of Glauber gluon exchanges, and $m$ is the index corresponding to the Feynman diagram in question. We perform each integral exactly, without assuming a soft gluon approximation for radiative energy loss. However, from the exact results we can easily take the soft gluon limit.

\section{D.1 Jet broadening}

The longitudinal momentum integral that appears in the single Born diagram for quark or gluon jet broadening equals to the following expression:

$$
I_{1}^{(1)}=\int \frac{d q_{1}^{-}}{2 \pi} \mathrm{e}^{i q_{1}^{-} \delta z_{1}} \Delta_{g}\left(p, q_{1}\right)=\int \frac{d q_{1}^{-}}{2 \pi} \mathrm{e}^{i q_{1}^{-} \delta z_{1}} \frac{1}{\omega_{1}-q_{1}^{-}}=-i \mathrm{e}^{i \omega_{1} \delta z_{1}}
$$

where the inverse formation time $\omega_{1}$ is defined according to Eq. (5.8) and Eq. (5.9):

$$
\omega_{1}=\Omega\left(p, q_{1}\right)=p^{-}-\frac{\left(\boldsymbol{p}_{\perp}-\boldsymbol{q}_{1 \perp}\right)^{2}-i \varepsilon}{p^{+}} .
$$

The double Born longitudinal integral looks like:

$$
\begin{aligned}
I_{1}^{(2)} & =\int \frac{d q_{1}^{-}}{2 \pi} \frac{d q_{2}^{-}}{2 \pi} \mathrm{e}^{i\left(q_{1}^{-} \delta z_{1}+q_{2}^{-} \delta z_{2}\right)} \Delta_{g}\left(p, q_{2}\right) \Delta_{g}\left(p, q_{1}+q_{2}\right) \\
& =(-i) \int \frac{d q_{2}^{-}}{(2 \pi)} \mathrm{e}^{i\left(\left(\omega_{12}-q_{2}^{-}\right) \delta z_{1}+q_{2}^{-} \delta z_{2}\right)} \Delta_{g}\left(p, q_{2}\right)=-\mathrm{e}^{i\left(\omega_{12} \delta z_{1}+\omega_{2}\left(\delta z_{2}-\delta z_{1}\right)\right)}
\end{aligned}
$$

where inverse formation time $\omega_{12}$ equals:

$$
\omega_{12}=\Omega\left(p, q_{1}+q_{2}\right)=p^{-}-\frac{\left(\boldsymbol{p}_{\perp}-\boldsymbol{q}_{1 \perp}-\boldsymbol{q}_{2 \perp}\right)^{2}-i \varepsilon}{p^{+}},
$$

and $\omega_{2}$ is identical to $\omega_{1}$ up to $q_{1} \leftrightarrow q_{2}$. In deriving Eq. (D.1) we used the fact that $\omega_{1}$ is in the upper complex plane, which we should choose, since $\delta z_{1}>0$. Similarly in first and second step in deriving Eq. (D.3) we used that $\omega_{12}$ and $\omega_{2}$ are in the upper $q^{-}$complex plane. In the second step we also used that $\delta z_{2}>\delta z_{1}$, 
which is true for second order in opacity diagram (we use time ordered notation $z_{0}<z_{1}<z_{2}<\ldots$ ). Next, we calculate the contact limit of the integral $I_{1}^{(2)}$, when $\delta z_{2}=\delta z_{1}+0$ :

$$
\begin{aligned}
& I_{1}^{(2 c)}=\int \frac{d q_{1}^{-}}{2 \pi} \frac{d q_{2}^{-}}{2 \pi} \mathrm{e}^{i\left(q_{1}^{-}+q_{2}^{-}\right) \delta z_{1}} \Delta_{g}\left(p, q_{2}\right) \Delta_{g}\left(p, q_{1}+q_{2}\right)=(-i) \int \frac{d q_{2}^{-}}{2 \pi} e^{i\left(\omega_{12}-q_{2}^{-}+q_{2}^{-}\right) \delta z_{1}} \Delta_{g}\left(p, q_{2}\right) \\
& =-i \mathrm{e}^{i \omega_{12} \delta z_{1}} \int \frac{d q_{2}^{-}}{2 \pi} \frac{1}{\omega_{2}-q_{2}^{-}}=\frac{i \mathrm{e}^{i \omega_{12} \delta z_{1}}}{2 \pi}\left(\ln \left(\infty-\omega_{2}\right)-\ln \left(-\infty-\omega_{2}\right)\right)=-\frac{1}{2} \mathrm{e}^{i \omega_{12} \delta z_{1}} .
\end{aligned}
$$

As one can see, in the second step in deriving Eq. (D.5) we cannot use Cauchy's theorem to perform the remaining $q_{2}^{-}$integral, since the boundary term at infinity does not vanish. Instead, we perform the integral directly. We find:

$$
I_{1}^{(2 c)}=\frac{1}{2} I_{1}^{(2)}\left(\delta z_{2}=\delta z_{1}\right)
$$

\section{D.2 Radiative energy loss}

The longitudinal momentum integrals that appear in the first three single Born diagrams on figure 5 are: ${ }^{17}$

$$
\begin{aligned}
I_{1}^{(1)} & =\int \frac{d q_{1}^{-}}{2 \pi} \mathrm{e}^{i q_{1}^{-} \delta z_{1}} \Delta_{g}\left(p+k, q_{1}\right), \\
I_{2}^{(1)} & =\int \frac{d q_{1}^{-}}{2 \pi} \mathrm{e}^{i q_{1}^{-} \delta z_{1}} \Delta_{g}\left(p, q_{1}\right) \Delta_{g}\left(p+k, q_{1}\right), \\
I_{3}^{(1)} & =\int \frac{d q_{1}^{-}}{2 \pi} \mathrm{e}^{i q_{1}^{-} \delta z_{1}} \Delta_{g}\left(k, q_{1}\right) \Delta_{g}\left(p+k, q_{1}\right) .
\end{aligned}
$$

These three integrals are functions of three inverse formation times, all given by the poles in Eq. (5.9) of propagator in Eq. (5.8):

$$
\begin{aligned}
& \Omega_{1}=\Omega\left(p+k, q_{1}\right)=p^{-}+k^{-}-\frac{\left(\boldsymbol{p}_{\perp}+\boldsymbol{k}_{\perp}-\boldsymbol{q}_{1 \perp}\right)^{2}-i \varepsilon}{p^{+}+k^{+}} \\
& \Omega_{2}=\Omega\left(p, q_{1}\right)=p^{-}-\frac{\left(\boldsymbol{p}_{\perp}-\boldsymbol{q}_{1 \perp}\right)^{2}-i \varepsilon}{p^{+}} \\
& \Omega_{3}=\Omega\left(k, q_{1}\right)=k^{-}-\frac{\left(\boldsymbol{k}_{\perp}-\boldsymbol{q}_{1 \perp}\right)^{2}-i \varepsilon}{k^{+}}
\end{aligned}
$$

The first integral in Eq. (D.7) we already derived in Eq. (D.1), where we need to substitute $\omega_{1} \rightarrow \Omega_{1}$. The integrals $I_{2}^{(1)}$ and $I_{3}^{(1)}$ can be reduced to the same integral in Eq. (D.1) by using the following trivial identity, which follows directly from definitions in Eq. (5.8) and Eq. (5.9):

$$
\Delta_{g}\left(p_{1}, q_{1}\right) \Delta_{g}\left(p_{2}, q_{1}\right)=-\frac{\Delta_{g}\left(p_{1}, q_{1}\right)-\Delta_{g}\left(p_{2}, q_{1}\right)}{\Omega\left(p_{1}, q_{1}\right)-\Omega\left(p_{2}, q_{1}\right)} .
$$

Note that in Eq. (D.13) the numerator depends on $q_{1}^{-}$, while the denominator does not. Thus, integrals $I_{2}^{(1)}$ and $I_{3}^{(1)}$ become a combination of two integrals in Eq. (D.1). As a result, we get the following exact expressions:

$$
\begin{aligned}
& I_{1}^{(1)}=-i \mathrm{e}^{i \Omega_{1} \delta z_{1}}, \\
& I_{2}^{(1)}=\frac{i}{\Omega_{1}-\Omega_{2}}\left(\mathrm{e}^{i \Omega_{1} \delta z}-\mathrm{e}^{i \Omega_{2} \delta z_{1}}\right), \\
& I_{3}^{(1)} \equiv \frac{i}{\Omega_{1}-\Omega_{3}}\left(\mathrm{e}^{i \Omega_{1} \delta z}-\mathrm{e}^{i \Omega_{3} \delta z_{1}}\right) .
\end{aligned}
$$

\footnotetext{
${ }^{17}$ The remaining two vanish when one takes the physical emitted gluon polarization vector.
} 
The soft gluon emission approximation corresponds to $x \ll 1$ and is of interest in sections 5 and 6 . In this limit the inverse formation times $\Omega_{1,2,3}$ reduce to only two non-trivial functions $\omega_{0}, \omega_{1}$. The corresponding approximate results read:

$$
\begin{aligned}
& \Omega_{1} \approx \frac{\boldsymbol{k}_{\perp}^{2}}{x p_{0}^{+}} \equiv \omega_{0}, \quad \Omega_{2} \approx 0, \quad \Omega_{3}=\frac{\boldsymbol{k}_{\perp}^{2}-\left(\boldsymbol{k}_{\perp}-\boldsymbol{q}_{1 \perp}\right)^{2}}{x p_{0}^{+}} \equiv \omega_{0}-\omega_{1}, \\
& I_{1}^{(1)} \approx-i \mathrm{e}^{i \omega_{0} \delta z_{1}}, \quad I_{2}^{(1)} \approx \frac{i}{\omega_{0}}\left[\mathrm{e}^{i \omega_{0} \delta z_{1}}-1\right], \quad I_{3}^{(1)} \approx i \frac{\mathrm{e}^{i \omega_{0} \delta z_{1}}}{\omega_{1}}\left[1-\mathrm{e}^{-i \omega_{1} \delta z_{1}}\right] .
\end{aligned}
$$

Next we move to the integrals in two Glauber exchange diagrams and their contact limits. The longitudinal momentum integrals that appear in the first six diagrams in figure 6 equal: ${ }^{18}$

$$
\begin{aligned}
I_{1}^{(2)} & =\int \frac{d q_{1}^{-}}{2 \pi} \frac{d q_{2}^{-}}{2 \pi} \mathrm{e}^{i\left(q_{1}^{-} \delta z_{1}+q_{2}^{-} \delta z_{2}\right)} \Delta_{g}\left(p+k, q_{2}\right) \Delta_{g}\left(p+k, q_{1}+q_{2}\right), \\
I_{2}^{(2)} & =\int \frac{d q_{1}^{-}}{2 \pi} \frac{d q_{2}^{-}}{2 \pi} \mathrm{e}^{i\left(q_{1}^{-} \delta z_{1}+q_{2}^{-} \delta z_{2}\right)} \Delta_{g}\left(p, q_{2}\right) \Delta_{g}\left(p, q_{1}+q_{2}\right) \Delta_{g}\left(p+k, q_{1}+q_{2}\right), \\
I_{3}^{(2)} & =\int \frac{d q_{1}^{-}}{2 \pi} \frac{d q_{2}^{-}}{2 \pi} \mathrm{e}^{i\left(q_{1}^{-} \delta z_{1}+q_{2}^{-} \delta z_{2}\right)} \Delta_{g}\left(k, q_{2}\right) \Delta_{g}\left(k, q_{1}+q_{2}\right) \Delta_{g}\left(p+k, q_{1}+q_{2}\right), \\
I_{4}^{(2)} & =\int \frac{d q_{1}^{-}}{2 \pi} \frac{d q_{2}^{-}}{2 \pi} \mathrm{e}^{i\left(q_{1}^{-} \delta z_{1}+q_{2}^{-} \delta z_{2}\right)} \Delta_{g}\left(p, q_{2}\right) \Delta_{g}\left(k, q_{1}\right) \Delta_{g}\left(p+k, q_{1}+q_{2}\right), \\
I_{5}^{(2)} & =\int \frac{d q_{1}^{-}}{2 \pi} \frac{d q_{2}^{-}}{2 \pi} \mathrm{e}^{i\left(q_{1}^{-} \delta z_{1}+q_{2}^{-} \delta z_{2}\right)} \Delta_{g}\left(p, q_{2}\right) \Delta_{g}\left(p+k, q_{2}\right) \Delta_{g}\left(p+k, q_{1}+q_{2}\right), \\
I_{6}^{(2)} & =\int \frac{d q_{1}^{-}}{2 \pi} \frac{d q_{2}^{-}}{2 \pi} \mathrm{e}^{i\left(q_{1}^{-} \delta z_{1}+q_{2}^{-} \delta z_{2}\right)} \Delta_{g}\left(k, q_{2}\right) \Delta_{g}\left(p+k, q_{2}\right) \Delta_{g}\left(p+k, q_{1}+q_{2}\right) .
\end{aligned}
$$

The corresponding integrals contain seven inverse formation times, which later after taking the contact limit and averaging over the medium states reduce to only two inverse formation times. We define the following seven poles $\alpha_{i}$ :

$$
\begin{aligned}
& \alpha_{1}=\Omega\left(p+k, q_{2}\right), \quad \alpha_{2}=\Omega\left(p+k, q_{1}+q_{2}\right), \quad \alpha_{3}=\Omega\left(p, q_{2}\right), \quad \alpha_{4}=\Omega\left(p, q_{1}+q_{2}\right), \\
& \alpha_{5}=\Omega\left(k, q_{2}\right), \quad \alpha_{6}=\Omega\left(k, q_{1}+q_{2}\right), \quad \alpha_{7}=\Omega\left(k, q_{1}\right) .
\end{aligned}
$$

Next we note that all double Born integrals except for $I_{4}^{(2)}$ can be found using combination of already calculated integral in Eq. (D.3) and the identity in Eq. (D.13). The results are following:

$$
\begin{aligned}
& I_{1}^{(2)}=-\mathrm{e}^{i\left(\alpha_{2} \delta z_{1}+\alpha_{1}\left(\delta z_{2}-\delta z_{1}\right)\right)}, \quad I_{1}^{(2 c)}=\frac{1}{2} I_{1}^{(2)}\left(\delta z_{2}=\delta z_{1}\right), \\
& I_{2}^{(2)}=\frac{\mathrm{e}^{i \alpha_{3}\left(\delta z_{2}-\delta z_{1}\right)}\left(\mathrm{e}^{i \alpha_{4} \delta z_{1}}-\mathrm{e}^{i \alpha_{2} \delta z_{1}}\right)}{\alpha_{4}-\alpha_{2}}, \quad I_{2}^{(2 c)}=\frac{1}{2} I_{2}^{(2)}\left(\delta z_{2}=\delta z_{1}\right), \\
& I_{3}^{(2)}=\frac{\mathrm{e}^{i \alpha_{5}\left(\delta z_{2}-\delta z_{1}\right)}\left(\mathrm{e}^{i \alpha_{6} \delta z_{1}}-\mathrm{e}^{i \alpha_{2} \delta z_{1}}\right)}{\alpha_{6}-\alpha_{2}}, \quad I_{3}^{(2 c)}=\frac{1}{2} I_{3}^{(2)}\left(\delta z_{2}=\delta z_{1}\right), \\
& I_{5}^{(2)}=\frac{\mathrm{e}^{i \alpha_{2} \delta z_{1}}\left(\mathrm{e}^{i \alpha_{3}\left(\delta z_{2}-\delta z_{1}\right)}-\mathrm{e}^{i \alpha_{1}\left(\delta z_{2}-\delta z_{1}\right)}\right)}{\alpha_{3}-\alpha_{1}}, \quad I_{5}^{(2 c)}=0, \\
& I_{6}^{(2)}=\frac{\mathrm{e}^{i \alpha_{2} \delta z_{1}}\left(\mathrm{e}^{i \alpha_{5}\left(\delta z_{2}-\delta z_{1}\right)}-\mathrm{e}^{i \alpha_{1}\left(\delta z_{2}-\delta z_{1}\right)}\right)}{\alpha_{5}-\alpha_{1}}, \quad I_{6}^{(2 c)}=0 .
\end{aligned}
$$

\footnotetext{
${ }^{18}$ The remaining three amplitudes in the figure 6 vanish.
} 
The final integral $I_{4}^{(2)}$ we just work out:

$$
I_{4}^{(2)}=\int \frac{d q_{1}^{-}}{2 \pi} \frac{d q_{2}^{-}}{2 \pi} \frac{\mathrm{e}^{i\left(q_{1}^{-} \delta z_{1}+q_{2}^{-} \delta z_{2}\right)}}{\left(\alpha_{3}-q_{2}^{-}\right)\left(\alpha_{7}-q_{1}^{-}\right)\left(\alpha_{2}-q_{1}^{-}-q_{2}^{-}\right)}=\int \frac{d q_{2}^{-}}{2 \pi} \frac{\mathrm{e}^{i q_{2}^{-} \delta z_{2}}}{\left(\alpha_{3}-q_{2}^{-}\right)} \frac{i\left(\mathrm{e}^{i \alpha_{7} \delta z_{1}}-\mathrm{e}^{i\left(\alpha_{2}-q_{2}^{-}\right) \delta z_{1}}\right)}{\alpha_{7}-\alpha_{2}+q_{2}^{-}},
$$

where in the first term of the remaining $q_{2}^{-}$integral we have to close the contour in the upper complex plane, while in the second one we close above for $\delta z_{2}>\delta z_{1}$ and below in the opposite case. Also, we know that $\alpha_{3}$ is in the positive complex plane, but we have to figure out the sign of imaginary part of $\left(\alpha_{7}-\alpha_{2}\right)$ :

$$
\operatorname{Im}\left(\alpha_{7}-\alpha_{2}\right)=\frac{\varepsilon}{k^{+}}-\frac{\varepsilon}{p^{+}+k^{+}}=\frac{\varepsilon(1-x)}{p_{0}^{+}}>0 .
$$

Thus, for physical momenta $p, k$, the second denominator in the last integral in Eq. (D.31) has a pole in the negative complex plane. With this in mind, we find the $I_{4}^{(2)}$ for two cases of interest:

$$
\begin{aligned}
& I_{4}^{(2)}\left(\delta z_{2}>\delta z_{1}\right)=\frac{\mathrm{e}^{i\left(\alpha_{3} \delta z_{2}+\alpha_{7} \delta z_{1}\right)}-\mathrm{e}^{i\left(\alpha_{2} \delta z_{1}+\alpha_{3}\left(\delta z_{2}-\delta z_{1}\right)\right)}}{\alpha_{7}-\alpha_{2}+\alpha_{3}}, \\
& I_{4}^{(2)}\left(\delta z_{2}<\delta z_{1}\right)=\frac{\mathrm{e}^{i\left(\alpha_{3} \delta z_{2}+\alpha_{7} \delta z_{1}\right)}-\mathrm{e}^{i\left(\alpha_{2} \delta z_{1}+\left(\alpha_{2}-\alpha_{7}\right)\left(\delta z_{2}-\delta z_{1}\right)\right)}}{\alpha_{7}-\alpha_{2}+\alpha_{3}} .
\end{aligned}
$$

Finally, taking the contact limit of the last integration in Eq. (D.31) we get:

$$
I_{4}^{(2 c)}=I_{4}^{(2)}\left(\delta z_{2}=\delta z_{1}+0\right)=I_{4}^{(2)}\left(\delta z_{2}=\delta z_{1}-0\right) .
$$

Next, in the contact limit and taking the average over medium, we have $\boldsymbol{q}_{1 \perp}+\boldsymbol{q}_{2 \perp}=\mathbf{0}$. This makes some of the $\alpha_{i}$ trivial. Directly from their definition we get:

$$
\alpha_{2}=\frac{(p+k)^{2}}{p_{0}^{+}}, \quad \alpha_{4}=\frac{p^{2}}{p^{+}}=0, \quad \alpha_{6}=\frac{k^{2}}{k^{+}}=0 .
$$

Taking these equations into account we list all the contact limit integrals:

$$
\begin{aligned}
& I_{1}^{(2 c)}=-\frac{1}{2} \mathrm{e}^{i \alpha_{2} \delta z_{1}}, \\
& I_{2}^{(2 c)}=\frac{1}{2} \frac{\mathrm{e}^{i \alpha_{2} \delta z_{1}}-1}{\alpha_{2}}, \\
& I_{3}^{(2 c)}=\frac{1}{2} \frac{\mathrm{e}^{i \alpha_{2} \delta z_{1}}-1}{\alpha_{2}}, \\
& I_{4}^{(2 c)}=\frac{\mathrm{e}^{i \alpha_{2} \delta z_{1}}}{\alpha_{7}-\alpha_{2}+\alpha_{3}}\left(\mathrm{e}^{i\left(\alpha_{7}-\alpha_{2}+\alpha_{3}\right) \delta z_{1}}-1\right), \\
& I_{5}^{(2 c)}=0, \\
& I_{6}^{(2 c)}=0 .
\end{aligned}
$$

As discussed above, the seven inverse formation times reduced to only two combinations after averaging over the medium. We define these two inverse formation times in the following way:

$$
\Omega_{4}=\alpha_{2}, \quad \Omega_{5}=\alpha_{7}+\alpha_{3} .
$$

Note that up until now we never used the soft gluon approximation, and all integrals and contact limits were exact. Finally, taking the soft gluon approximation gives:

$$
\Omega_{4} \approx \omega_{0}, \quad \Omega_{5} \approx \omega_{0}-\omega_{1} .
$$




\section{E. $T$ Wilson line}
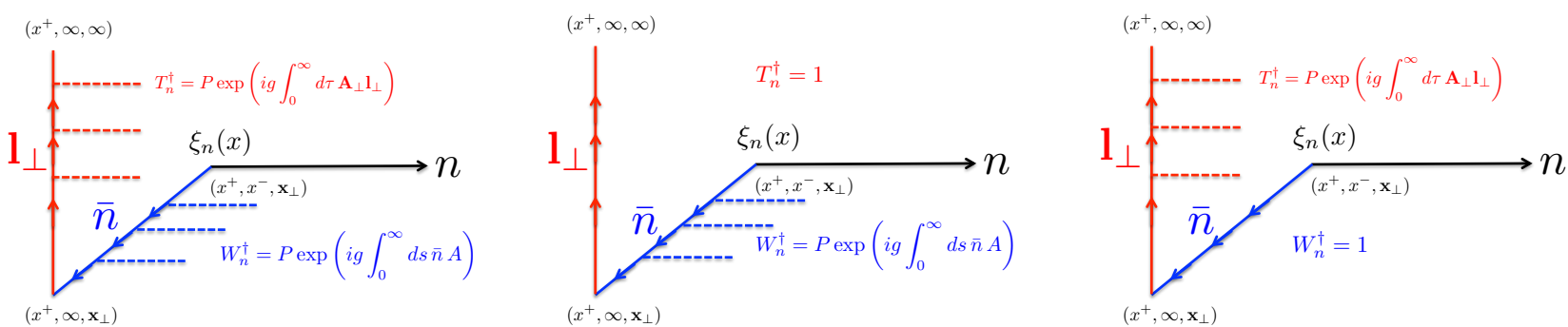

Figure 12: The collinear and transverse gauge links. Left: the generic gauge, middle: regular covariant gauge and right: singular light-cone gauge.

Wilson lines are a common ingredient for building gauge-invariant objects in field theory. For example, the collinear Wilson line $W_{n}$ is used in SCET to dress the collinear quark field $\xi_{n}$ to form a gauge-invariant jet field $\chi_{n}=W_{n}^{\dagger} \xi_{n}$. However, in the light-cone gauge the collinear Wilson line disappears $W_{n}^{\dagger}=1$. In the context of SDIS it has been realized [60], [61] that additional transverse gauge link is required in this gauge in order to describe the final state interactions in a gauge invariant way.

More recently, it has been proposed that SCET has to be expanded [54] by including a transverse gauge link for gauge invariance of certain non-perturbative matrix elements, like the transverse momentumdependent parton distribution functions. Also, it has been shown in Ref. [54] that the SCET jet function calculated in the light-cone gauge in the presence of the transverse gauge link, which the authors denote as the transverse Wilson line $T_{n}^{\dagger}$, is independent of the light-cone prescription, which unambiguously cancels between the gluon propagator and the gauge link, and leads to a result which is the same as in the covariant gauge.

$$
\begin{aligned}
& \chi_{n, p}=T_{n}^{\dagger} W_{n}^{\dagger} \xi_{n, p}, \\
& T_{n}^{\dagger}=\mathcal{P} \exp \left(i g \int_{0}^{\infty} d \tau \boldsymbol{A}_{\perp} \cdot \boldsymbol{l}_{\perp}\right),
\end{aligned}
$$

where $\mathcal{P}$ denotes the path ordering. As one can see from the figure 12 the effect of the transverse gauge link vanishes in the covariant gauge, since the gauge field is zero at infinity, while in the singular light-cone gauge this gauge link is non-zero depending on the boundary conditions, similar to the gluon propagator prescription in the light-cone gauge.

Our calculation of the single and double Born amplitudes in the light-cone gauge in $\mathrm{SCET}_{\mathrm{G}}$ in the absence of the $T_{n}$ Wilson line leads to the same result as in the covariant gauge, but only in the $-i \varepsilon$ prescription. In all other prescriptions we get a different result, as one can see from Eq. (7.7) and table 7.2. In this appendix we show that once the $T_{n}$ Wilson line is introduced into the calculation, the prescription dependence cancels and the gauge invariance is restored similarly to the result in [54] for the SCET jet function calculated in the light-cone gauge. In the remainder of this section we assume the light-cone gauge, so $W_{n}=1$ and we derive the Feynman rules for $T_{n}$ Wilson line emission.

A useful expression for the propagator of the gluon emitted from the transverse gauge link was derived in [54] and we show it in Figure 13, where the prescription dependence is encoded into the number $C_{\infty}^{\text {(Pres) }}$. 


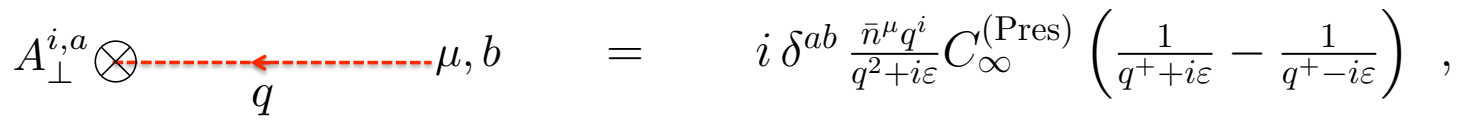

Figure 13: The propagator of the $T$ Wilson line emitted gluon.

Its dependence on the prescription is given in the table E. Note that the difference of this table and the propagator in figure 13 from [54] is due to the different notation of ingoing vs outgoing momentum flow into the vertex.

\begin{tabular}{||c||c||c|}
\hline Prescription & $\frac{1}{\left[q^{+}\right]}$ & $C_{\infty}^{\text {(Pres) }}$ \\
\hline \hline$+i \varepsilon$ & $\frac{1}{q^{+}+i \varepsilon}$ & 1 \\
$-i \varepsilon$ & $\frac{1}{q^{+}-i \varepsilon}$ & 0 \\
$\mathrm{PV}$ & $\frac{1}{2}\left(\frac{1}{q^{+}+i \varepsilon}+\frac{1}{q^{+}-i \varepsilon}\right)$ & $\frac{1}{2}$ \\
$\mathrm{ML}$ & $\frac{1}{q^{+}+i \varepsilon \operatorname{sign}\left(q^{-}\right)}$ & $\theta\left(q^{-}\right)$ \\
\hline
\end{tabular}

Table 3: Dependence of $C_{\infty}^{(\text {Pres })}$ on the light-cone prescription.

First, from the form of the propagator in figure 13 one can see that the $T_{n}$ Wilson line cannot produce physical gluons in the final state, since $\bar{n} \cdot \varepsilon=0$ in the light-cone gauge. However, this propagator contracted with the static source term $v_{\mu}$ doesn't vanish and is leading order in the effective theory power counting. In order to derive the Feynman rules of $T_{n}$ emission from the quark line we use the definition of the gauge invariant quark field Eq. (E.1) and the explicit expression for the transverse gauge link in Eq. (E.2). Finally we include the propagator in figure 13 to obtain first two Feynman rules in the figure 14 below.

In order to derive similar Feynman rules with the gluon line we need the expression of the gauge invariant gluon field which is a straightforward generalization of the previous definition Eq. (4.19), now including the $T$ Wilson line:

$$
\mathcal{B}_{n}^{\mu}=\frac{1}{g}\left[T_{n}^{\dagger} W_{n}^{\dagger}\left(i \partial_{n}^{\mu}+g A_{n}^{\mu}\right) W_{n} T_{n}\right]
$$

where the square brackets indicate that the derivative acts only within the brackets. From the expression Eq. (E.3) and again using the definition Eq. (E.2) and the propagator from figure 13 we obtain the last two Feynman rules in figure 14. 


$$
\begin{aligned}
& \underset{q}{p}=(b)_{r}(b)_{T_{i}} v\left(q_{\perp}\right) C_{\infty}^{(\mathrm{Pres})}\left(\frac{1}{q^{+}+i \varepsilon}-\frac{1}{q^{+}-i \varepsilon}\right) \\
& q _ { 1 } \longdiv { q _ { 2 } } = - \frac { 1 } { 2 } \frac { l _ { \perp } q _ { 1 \perp } ( b _ { 1 } b _ { 2 } ) _ { r } + l _ { \perp } q _ { 2 \perp } ( b _ { 2 } b _ { 1 } ) _ { r } } { l _ { \perp } q _ { 1 \perp } + l _ { \perp } q _ { 2 } } ( b _ { 1 } ) _ { T _ { i } } ( b _ { 2 } ) _ { T _ { j } } v ( q _ { 1 \perp } ) v ( q _ { 2 \perp } ) \\
& \times\left[C_{\infty}^{(\text {Pres })}\right]^{2}\left(\frac{1}{q_{1}^{+}+i \varepsilon}-\frac{1}{q_{1}^{+}-i \varepsilon}\right)\left(\frac{1}{q_{2}^{+}+i \varepsilon}-\frac{1}{q_{2}^{+}-i \varepsilon}\right) \\
& \underset{\uparrow q}{\stackrel{p}{\longrightarrow} \rightarrow-a}=-i f^{a b c}\left(-g_{\perp}^{\mu \nu}\right)(c)_{r}(b)_{T_{i}} v\left(q_{\perp}\right) C_{\infty}^{(\text {Pres })}\left(\frac{1}{q^{+}+i \varepsilon}-\frac{1}{q^{+}-i \varepsilon}\right) \\
& q_{1} \stackrel{p}{q_{2}}=-\frac{1}{2}\left(-g_{\perp}^{\mu \nu}\right) \frac{l_{\perp} q_{1 \perp} f^{a b_{1} c_{1}} f^{c_{1} b_{2} e}+l_{\perp} q_{2 \perp} f^{a b_{2} c_{1} f^{c_{1} b_{1} e}}}{l_{\perp} q_{1 \perp}+l_{\perp} q_{2 \perp}}(e)_{r}\left(b_{1}\right)_{T_{i}}\left(b_{2}\right)_{T_{j}} v\left(q_{1 \perp}\right) v\left(q_{2 \perp}\right) \\
& \times\left[C_{\infty}^{(\text {Pres })}\right]^{2}\left(\frac{1}{q_{1}^{+}+i \varepsilon}-\frac{1}{q_{1}^{+}-i \varepsilon}\right)\left(\frac{1}{q_{2}^{+}+i \varepsilon}-\frac{1}{q_{2}^{+}-i \varepsilon}\right)
\end{aligned}
$$

Figure 14: Feynman rules for the gluon emission from the transverse Wilson line $T_{n}$ for single and double gluon emission from a quark and a gluon line.

\section{References}

[1] G. Sterman and S. Weinberg, Jets from Quantum Chromodynamics, Phys. Rev. Lett. 39 (1977) 1436.

[2] R. P. Feynman, R. D. Field, and G. C. Fox, Quantum-chromodynamic approach for the large-transversemomentum production of particles and jets, Phys. Rev. D18 (1978) 3320.

[3] J. R. Ellis, Beyond the standard model with the LHC, Nature 448 (2007) 297-301.

[4] The ATLAS Collaboration, G. Aad et al., Expected Performance of the ATLAS Experiment - Detector, Trigger and Physics, arXiv:0901.0512.

[5] CMS Collaboration, G. L. Bayatian et al., CMS technical design report, volume II: Physics performance, J. Phys. G34 (2007) 995-1579.

[6] J. C. Collins and G. F. Sterman, SOFT PARTONS IN QCD, Nucl. Phys. B185 (1981) 172.

[7] J. C. Collins, D. E. Soper, and G. F. Sterman, Factorization of Hard Processes in QCD, Adv. Ser. Direct. High Energy Phys. 5 (1988) 1-91, [hep-ph/0409313].

[8] C. W. Bauer, S. Fleming, and M. E. Luke, Summing Sudakov logarithms in B-i X/s gamma in effective field theory, Phys. Rev. D63 (2000) 014006, [hep-ph/0005275].

[9] C. W. Bauer, S. Fleming, D. Pirjol, and I. W. Stewart, An effective field theory for collinear and soft gluons: Heavy to light decays, Phys. Rev. D63 (2001) 114020, [hep-ph/0011336].

[10] C. W. Bauer and I. W. Stewart, Invariant operators in collinear effective theory, Phys. Lett. B516 (2001) 134-142, [hep-ph/0107001].

[11] C. W. Bauer, D. Pirjol, and I. W. Stewart, Soft-Collinear Factorization in Effective Field Theory, Phys. Rev. D65 (2002) 054022, [hep-ph/0109045]. 
[12] C. W. Bauer, S. Fleming, D. Pirjol, I. Z. Rothstein, and I. W. Stewart, Hard scattering factorization from effective field theory, Phys. Rev. D66 (2002) 014017, [hep-ph/0202088].

[13] A. V. Manohar, Deep inelastic scattering as $x-\dot{z} 1$ using soft-collinear effective theory, Phys. Rev. D68 (2003) 114019, [hep-ph/0309176].

[14] T. Becher, M. Neubert, and B. D. Pecjak, Factorization and momentum-space resummation in deep- inelastic scattering, JHEP 01 (2007) 076, [hep-ph/0607228].

[15] S. Fleming, A. H. Hoang, S. Mantry, and I. W. Stewart, Top Jets in the Peak Region: Factorization Analysis with NLL Resummation, Phys. Rev. D77 (2008) 114003, [arXiv:0711.2079].

[16] C. W. Bauer, S. P. Fleming, C. Lee, and G. F. Sterman, Factorization of e+e-Event Shape Distributions with Hadronic Final States in Soft Collinear Effective Theory, Phys. Rev. D78 (2008) 034027, [arXiv:0801.4569].

[17] T. Becher and M. D. Schwartz, Direct photon production with effective field theory, JHEP 02 (2010) 040, [arXiv:0911.0681].

[18] S. Mantry and F. Petriello, Factorization and Resummation of Higgs Boson Differential Distributions in Soft-Collinear Effective Theory, Phys. Rev. D81 (2010) 093007, [arXiv:0911.4135].

[19] I. W. Stewart, F. J. Tackmann, and W. J. Waalewijn, Factorization at the LHC: From PDFs to Initial State Jets, Phys. Rev. D81 (2010) 094035, [arXiv:0910.0467].

[20] S. Fleming, A. H. Hoang, S. Mantry, and I. W. Stewart, Jets from massive unstable particles: Top-mass determination, Phys. Rev. D77 (2008) 074010, [hep-ph/0703207].

[21] T. Becher, M. Neubert, and G. Xu, Dynamical Threshold Enhancement and Resummation in Drell- Yan Production, JHEP 07 (2008) 030, [arXiv:0710.0680].

[22] T. Becher and M. D. Schwartz, A Precise determination of $\alpha_{s}$ from LEP thrust data using effective field theory, JHEP 07 (2008) 034, [arXiv:0803.0342].

[23] V. Ahrens, T. Becher, M. Neubert, and L. L. Yang, Renormalization-Group Improved Prediction for Higgs Production at Hadron Colliders, Eur. Phys. J. C62 (2009) 333-353, [arXiv:0809.4283].

[24] A. Hornig, C. Lee, and G. Ovanesyan, Effective Predictions of Event Shapes: Factorized, Resummed, and Gapped Angularity Distributions, JHEP 05 (2009) 122, [arXiv:0901.3780].

[25] I. Vitev, S. Wicks, and B.-W. Zhang, A theory of jet shapes and cross sections: from hadrons to nuclei, JHEP 11 (2008) 093, [arXiv:0810.2807].

[26] I. Vitev and B.-W. Zhang, Jet tomography of high-energy nucleus-nucleus collisions at next-to-leading order, Phys. Rev. Lett. 104 (2010) 132001, [arXiv:0910.1090].

[27] T. Renk, Medium-modified Jet Shapes and other Jet Observables from in-medium Parton Shower Evolution, Phys. Rev. C80 (2009) 044904, [arXiv:0906.3397].

[28] R. B. Neufeld, I. Vitev, and B. W. Zhang, The physics of $Z^{0} / \gamma^{*}$-tagged jets at the LHC, arXiv:1006.2389.

[29] S. Salur, Full Jet Reconstruction in Heavy Ion Collisions, Nucl. Phys. A830 (2009) 139c-146c, [arXiv: 0907.4536].

[30] PHENIX Collaboration, Y.-S. Lai, Probing medium-induced energy loss with direct jet reconstruction in $p+p$ and $\mathrm{Cu}+\mathrm{Cu}$ collisions at PHENIX, Nucl. Phys. A830 (2009) 251c-254c, [arXiv:0907.4725].

[31] STAR Collaboration, M. Ploskon, Inclusive cross section and correlations of fully reconstructed jets in 200 GEV Au+Au and p+p collisions, Nucl. Phys. A830 (2009) 255c-258c, [arXiv:0908.1799]. 
[32] ALICE Collaboration Collaboration, K. Aamodt et al., Suppression of Charged Particle Production at Large Transverse Momentum in Central Pb-Pb Collisions at $\sqrt{s_{N N}}=2.76$ TeV, Phys.Lett. B696 (2011) 30-39, [arXiv:1012.1004]. * Temporary entry *.

[33] CMS Collaboration Collaboration, S. Chatrchyan et al., Observation and studies of jet quenching in $\mathrm{PbPb}$ collisions at nucleon-nucleon center-of-mass energy $=2.76 \mathrm{TeV}$, arXiv:1102.1957. * Temporary entry *.

[34] Atlas Collaboration Collaboration, G. Aad et al., Observation of a Centrality-Dependent Dijet Asymmetry in Lead-Lead Collisions at sqrt $(S(N N))=2.76 \mathrm{TeV}$ with the ATLAS Detector at the LHC, arXiv:1011.6182. * Temporary entry*.

[35] A. Idilbi and A. Majumder, Extending Soft-Collinear-Effective-Theory to describe hard jets in dense QCD media, Phys. Rev. D80 (2009) 054022, [arXiv:0808.1087].

[36] F. D'Eramo, H. Liu, and K. Rajagopal, Transverse Momentum Broadening and the Jet Quenching Parameter, Redux, arXiv:1006.1367.

[37] M. Gyulassy, P. Levai, and I. Vitev, Reaction operator approach to multiple elastic scatterings, Phys. Rev. D66 (2002) 014005, [nucl-th/0201078].

[38] J.-w. Qiu and I. Vitev, Transverse momentum diffusion and broadening of the back- to-back di-hadron correlation function, Phys. Lett. B570 (2003) 161-170, [nuc1-th/0306039].

[39] R. Baier, D. Schiff, and B. G. Zakharov, Energy loss in perturbative QCD, Ann. Rev. Nucl. Part. Sci. 50 (2000) 37-69, [hep-ph/0002198].

[40] M. Gyulassy, P. Levai, and I. Vitev, Reaction operator approach to non-Abelian energy loss, Nucl. Phys. B594 (2001) 371-419, [nucl-th/0006010].

[41] X.-N. Wang and X.-f. Guo, Multiple parton scattering in nuclei: Parton energy loss, Nucl. Phys. A696 (2001) 788-832, [hep-ph/0102230].

[42] P. B. Arnold, G. D. Moore, and L. G. Yaffe, Photon and Gluon Emission in Relativistic Plasmas, JHEP 06 (2002) 030, [hep-ph/0204343].

[43] M. Djordjevic, Theoretical formalism of radiative jet energy loss in a finite size dynamical QCD medium, Phys.Rev. C80 (2009) 064909, [arXiv:0903.4591].

[44] I. Stewart and I. Rothstein, Talk presented at scet2010 in ringberg, germany, .

[45] M. E. Luke, A. V. Manohar, and I. Z. Rothstein, Renormalization group scaling in nonrelativistic QCD, Phys. Rev. D61 (2000) 074025, [hep-ph/9910209].

[46] C. W. Bauer, B. O. Lange, and G. Ovanesyan, On Glauber modes in Soft-Collinear Effective Theory, arXiv:1010.1027.

[47] J. C. Collins, D. E. Soper, and G. Sterman, FACTORIZATION FOR ONE LOOP CORRECTIONS IN THE DRELL-YAN PROCESS, Nucl. Phys. B223 (1983) 381.

[48] G. T. Bodwin, Factorization of the Drell-Yan Cross-Section in Perturbation Theory, Phys. Rev. D31 (1985) 2616 [Erratum: Phys. Rev. D 34 (Dec, 1986), 3932].

[49] J. C. Collins, D. E. Soper, and G. F. Sterman, Factorization for Short Distance Hadron - Hadron Scattering, Nucl. Phys. B261 (1985) 104.

[50] L. F. Abbott, Introduction to the Background Field Method, Acta Phys. Polon. B13 (1982) 33.

[51] I. Vitev, Non-Abelian energy loss in cold nuclear matter, Phys.Rev. C75 (2007) 064906, [hep-ph/0703002].

[52] M. Baumgart, C. Marcantonini, and I. W. Stewart, Parton Shower with NLO Kinematic Power Corrections, arXiv:1007.0758. 
[53] C. W. Bauer, O. Cata, and G. Ovanesyan, On different ways to quantize Soft-Collinear Effective Theory, arXiv:0809.1099.

[54] A. Idilbi and I. Scimemi, Singular and Regular Gauges in Soft Collinear Effective Theory: The Introduction of the New Wilson Line T, arXiv:1009.2776. * Temporary entry *.

[55] I. Vitev and B.-W. Zhang, A Systematic study of direct photon production in heavy ion collisions, Phys.Lett. B669 (2008) 337-344, [arXiv:0804.3805].

[56] R. Sharma, I. Vitev, and B.-W. Zhang, Light-cone wave function approach to open heavy flavor dynamics in QCD matter, Phys.Rev. C80 (2009) 054902, [arXiv:0904.0032].

[57] B.-W. Zhang and X.-N. Wang, Multiple parton scattering in nuclei: Beyond helicity amplitude approximation, Nucl.Phys. A720 (2003) 429-451, [hep-ph/0301195].

[58] R. Neufeld, I. Vitev, and B.-W. Zhang, Toward a determination of the shortest radiation length in nature, arXiv: 1010.3708 .

[59] I. Vitev, A brief overview of fixed-order perturbative QCD calculations of jet production in heavy-ion collisions, arXiv:1010.5803. * Temporary entry *.

[60] X.-d. Ji and F. Yuan, Parton distributions in light cone gauge: Where are the final state interactions?, Phys.Lett. B543 (2002) 66-72, [hep-ph/0206057].

[61] A. V. Belitsky, X. Ji, and F. Yuan, Final state interactions and gauge invariant parton distributions, Nucl.Phys. B656 (2003) 165-198, [hep-ph/0208038]. 